\title{
Quantum geometry of elliptic Calabi-Yau manifolds
}

\author{
Albrecht Klemm, Jan Manschot and Thomas Wotschke
}

\begin{abstract}
We study the quantum geometry of the class of Calabi-Yau threefolds, which are elliptic fibrations over a two-dimensional toric base. A holomorphic anomaly equation for the topological string free energy is proposed, which is iterative in the genus expansion as well as in the curve classes in the base. $T$-duality on the fibre implies that the topological string free energy also captures the BPS-invariants of $D 4$-branes wrapping the elliptic fibre and a class in the base. We verify this proposal by explicit computation of the BPS invariants of $3 D 4$-branes on the rational elliptic surface.
\end{abstract}

1. Introduction

2. Classical geometry of elliptically fibred Calabi-Yau spaces

2.1. The classical geometrical data of elliptic fibrations

2.2. Realizations in toric ambient spaces

3. Quantum geometry of elliptic fibrations

3.1. Quantum cohomology, modularity and the anomaly equations

3.2. The $B$-model approach to elliptically fibred Calabi-Yau spaces

3.2.1 Examples: elliptic fibrations over $\mathbb{P}^{2}$ and $\mathbb{F}_{1}$.

4. Derivation of the holomorphic anomaly equation 
"CNTP-6-4-A5-KLEMM" — 2013/6/5 — 15:56 — page 850 — \#2

5. T-duality on the fibre

Example: $X_{18}[1,1,1,6,9]$

6. BPS invariants of the rational elliptic surface

6.1. The lattice $H^{2}\left(\mathbb{F}_{9}, \mathbb{Z}\right)$

6.2. BPS invariants for $r \leq 3$

Rank 1

Rank 2

896

Modularity

Rank 3

Appendix A. Toric data for the elliptic hypersurfaces

Appendix B. Results for the other fibre types with $\mathbb{F}_{1}$ base

909

Appendix C. Modular functions

\section{Introduction}

Topological string theory on local Calabi-Yau manifolds has been a remarkable success story. It counts the open and closed instantons corrections to topological numbers, which can be seen as an extension from classical geometry to quantum geometry. By now we can solve it in very different ways, namely by localisation, by direct integration of the holomorphic anomaly equations, by the topological vertex [1] or by the matrix model techniques in the remodelled $B$-model [12]. Topological string theory on local CalabiYau manifolds gives deep insights in the interplay between large $N$ gauge theory/string theory duality, mirror duality, the theory of modular forms and knot theory and is by geometric engineering [37] intimately related to the construction of effective $N=2$ and $N=1$ rigid supersymmetric theories in four dimensions.

On global, i.e., compact, Calabi-Yau manifolds, which give rise to $N=2$ and $N=1$ effective supergravity theories in four dimensions, the situation is 
less understood. Direct integration extends the theory of modular objects to the Calabi-Yau spaces and establishes that closed topological string amplitudes can be written as polynomial in modular objects, but the boundary conditions for the integration are in contrast to the local case not completely known. As an example, on the quintic surface the closed topological string can be solved up to genus 51 [33].

In [17] mirror symmetry was made local in the decompactification limit of fibred Calabi-Yau threefolds. Here we want to do the opposite and study how the quantum geometry extends from the local to the global case, when a class of local Calabi-Yau geometries is canonically compactified by an elliptic Calabi-Yau fibration with projection $\pi: M \rightarrow B$. This easy class of local to global pairs, will be described to a large extend by complete intersections in explicit toric realizations. As we review in Section 2, if the elliptic fibration has only $I_{1}$ fibres the classical cohomology of $M$ is completely determined by the classical intersection of the base $B$ and the number of sections, which depends on the Mordell Weyl group of the elliptic family.

The decisive question to which extend this holds for the quantum geometry is addressed in Section 3 using mirror symmetry. The instanton numbers are counted by (quasi)-modular forms of congruence subgroups of $\mathrm{SL}(2, \mathbb{Z})$ capturing curves with a fixed degree in the base for all degrees in the fibre. The weights of the forms depend on the genus and the base class. This structure has been discovered for elliptically fibred surfaces in [39] and for elliptically fibred threefolds in [40]. We establish here a holomorphic anomaly Equation (3.9) based on the non-holomorphic modular completion of the quasi-modular forms which is iterative in the genus, as in [9], and also in the base classes generalizing $[32,57]$.

Our construction can be viewed also as a step to a better understanding of periods and instanton corrections in $F$-theory compactifications and a preliminary study using the data of $[38,41,56]$ reveals that the structure at the relevant generera $g=0,1$ extends.

A holomorphic anomaly equation is also known to appear for generating functions of Bogomolny-Prasad-Sommerfield (BPS) invariants of higher dimensional $D$-branes, in particular $D 4$-branes on a surface $[2,20,49,53,59]$. Interestingly, on elliptic Calabi-Yau fibrations, double $T$-duality on the elliptic fibre (or Fourier-Mukai transform) $[3,4,7,57]$ transforms D2-branes wrapped on base classes into $D 4$-branes that also wrap the elliptic fibre and vice versa. The $D 4$-brane holomorphic anomaly is therefore related to the one of Gromov-Witten theory for these geometries. Moreover, the mirror periods provide predictions for D4-brane BPS invariants which correspond to those of (small) black holes in supergravity. 
We discuss higher dimensional branes on Calabi-Yau elliptic fibrations in Sections 5 and 6 . We compare the predictions from the periods for $D 4$-brane BPS invariants with existing methods in the literature for the computation of small charge BPS invariants [11, 16, 19,22,23,52,54]. The predictions of the periods are in many cases compatible with these methods. We leave a more precise study of $D 4$-brane BPS states on general elliptic fibrations to future work.

Section 6 specializes to the elliptic fibration over the Hirzebruch surface $\mathbb{F}_{1}$. The periods of its mirror geometry provide the BPS invariants of $D 4$-branes on the rational elliptic surface (also known as $\frac{1}{2} K_{3}$ ) as proposed originally by Minahan et al. [57]. We revisit and extend the verification of this proposal for $\leq 3 D$-branes using algebraic-geometric techniques $[25,26,51,53,55,60,62]$.

\section{Classical geometry of elliptically fibred Calabi-Yau spaces}

In this section, we study the classical geometry of elliptically fibered CalabiYau threefolds $M$ with base $B$ and projection map $\pi: M \rightarrow B$. We provide expressions for the Chern classes as well as the construction of such Calabi-Yau three manifolds by means of toric geometry. Elliptic fibrations are locally described by a Weierstrass form

$$
y^{2}=4 x^{3}-x w^{4} g_{2}(\underline{u})-g_{3}(\underline{u}) w^{6},
$$

where $\underline{u}$ are coordinates on the base $B$. A global description can be defined by an embedding as a hypersurface or complete intersection in an ambient space $W$. Explicitly, we consider cases, which allow a representation as a hypersurface or complete intersection in a toric ambient space. We restrict our attention to the case where the fibre degenerations are only of Kodaira type $I_{1}$, which means that the discriminant $\Delta=g_{2}^{3}-27 g_{3}^{2}$ of (2.1) has only simple zeros on $B$, which are not simultaneously zeros of $g_{2}$ and $g_{3}$. It was observed in [38] that such tame fibrations can be constructed torically over toric bases, which are given themselves defined by reflexive polyhedra. These tame fibrations are not enough to address immediately phenomenological interesting models in F-theory, due to the lack of non-abelian gauge symmetry in the effective four-dimensional physics, which come precisely from more singular fibres in the Kodaira classification. However, we note that the examples discussed here have a particular large number of complex moduli. 
Adjusting the latter and blowing up the singularities, not necessarily torically, is a more local operation, at least of co-dimension one in the base, which can be addressed in a second step.

\subsection{The classical geometrical data of elliptic fibrations}

Let $W \rightarrow B$ be a fibre bundle whose fibre is an $r-1$ dimensional weighted projective space $\mathbb{P}\left(w_{1}, \ldots, w_{r}\right)$ and $B$ an almost toric Fano surface. We define elliptically fibred Calabi-Yau threefolds $M \rightarrow B$ as hypersurfaces or complete intersections in $W$. We consider the following choices of weights

$$
\left(w_{1}, \ldots, w_{r}\right)=\{(1,2,3),(1,1,2),(1,1,1),(1,1,1,1)\} .
$$

In particular, the elliptic fibres are degree $6,4,3$ hypersurfaces and a bidegree $(2,2)$ complete intersection in the coordinates of the given weighted projective space. In the case of rational elliptic surfaces these fibres lead to $E_{8}, E_{7}, E_{6}$ and $D_{5}$ del Pezzo surfaces, named so as the integers cohomology lattice of the surface contains the intersection form of the Cartan-matrix of the corresponding Lie algebras. In the following we keep these names for the fibration types.

Let us discuss the first case. This leads canonically to an embedding with a single section; however, most of the discussion below applies to the other cases with minor modifications. Denote by $\alpha=c_{1}(\mathcal{O}(1))$ with $\mathcal{O}(1)$ the line bundle on $W$ induced by the hyperplane class of the projective fibre and $K=-c_{1}$ the canonical bundle of the base.

The coordinates $w, x, y$ are sections of $\mathcal{O}(1), \mathcal{O}(1)^{2} \otimes K^{-2}$ and $\mathcal{O}(1)^{3} \otimes$ $K^{-3}$ while $g_{2}$ and $g_{3}$ are sections of $K^{-4}$ and $K^{-6}$ respectively so that (2.1) is a section of $\mathcal{O}(1)^{6} \otimes K^{-6}$. The corresponding divisors $w=0, x=0, y=0$ have no intersection, i.e., $\alpha\left(\alpha+c_{1}\right)\left(\alpha+c_{1}\right)=0$ in the cohomology ring of $W$ and

$$
\alpha\left(\alpha+c_{1}\right)=0
$$

in the cohomology ring of $M$. Let us assume that the discriminant $\Delta$ vanishes for generic complex moduli only to first order in the coordinates of $B$ at loci, which are not simultaneously zeros of $g_{2}$ and $g_{3}$. In this case, its class must satisfy

$$
[\Delta]=c_{1}(B)=-K
$$


Table 1: Chern classes $\mathcal{C}_{i}$ of regular elliptic Calabi-Yau manifolds. Integrating $\alpha$ over the fibre yields a factor $a=\frac{\prod_{i} d_{i}}{\prod_{i} w_{i}}$, i.e., the number of sections $1,2,3,4$ for the three fibrations in turn.

\begin{tabular}{lccc}
\hline Fibre & $\mathcal{C}_{2}$ & $\mathcal{C}_{3}$ & $\mathcal{C}_{4}$ \\
\hline$E 8$ & $12 \alpha c_{1}+\left(11 c_{1}^{2}+c_{2}\right)$ & $-60 \alpha c_{1}^{2}-\left(60 c_{1}^{3}+c_{2} c_{1}-c_{3}\right)$ & $12 \alpha c_{1}\left(30 c_{1}^{2}+c_{2}\right)$ \\
$E 7$ & $6 \alpha c_{1}+\left(5 c_{1}^{2}+c_{2}\right)$ & $-18 \alpha c_{1}^{2}-\left(18 c_{1}^{3}+c_{2} c_{1}-c_{3}\right)$ & $6 \alpha c_{1}\left(12 c_{1}^{2}+c_{2}\right)$ \\
$E 6$ & $4 \alpha c_{1}+\left(3 c_{1}^{2}+c_{2}\right)$ & $-8 \alpha c_{1}^{2}-\left(8 c_{1}^{3}+c_{2} c_{1}-c_{3}\right)$ & $4 \alpha c_{1}\left(6 c_{1}^{2}+c_{2}\right)$ \\
$D 5$ & $3 \alpha c_{1}+\left(2 c_{1}^{2}+c_{2}\right)$ & $-4 \alpha c_{1}^{2}-\left(4 c_{1}^{3}+c_{2} c_{1}-c_{3}\right)$ & $3 \alpha c_{1}\left(3 c_{1}^{2}+c_{2}\right)$ \\
\hline
\end{tabular}

to obey the Calabi-Yau condition and the fibre over the vanishing locus of the discriminant is of Kodaira type $I_{1}$. For this generic fibration, the properties of $M$ only depend on the properties of $B$.

For example, using the adjunction formula and the relation (2.3) to reduce to linear terms in $\alpha$ allows us to write the total Chern class as ${ }^{1}$

$$
\mathcal{C}=\left(1+\sum_{i=1}^{d_{M}-1} c_{i}\right) \frac{(1+\alpha)\left(1+w_{2} \alpha+w_{2} c_{1}\right)\left(1+w_{3} \alpha+w_{3} c_{1}\right)}{1+d \alpha+d c_{1}}
$$

The Chern forms $\mathcal{C}_{k}$ of $M$ are the coefficients in the formal expansion of (2.5) of the degree $k$ in terms of $a$ and the monomials of the Chern forms $c_{i}$ of base $B$. The formulas (2.3) and (2.5) apply for all projectivisations.

In the following, the results for various dimensions $d_{M}$ are presented. For $d_{M}=2$, one gets from table 1 by integrating over the fibre in all cases $\chi(M)=12 \int_{B} c_{1}$ and $\mathbb{P}^{1}$ is the only admissible base. Similar for $d_{M}=3$ one gets for the different projectivisations $\chi(M)=-60 \int_{B} c_{1}^{2}, \chi(M)=-36 \int_{B} c_{1}^{2}$, $\chi(M)=-24 \int_{B} c_{1}^{2}$ and $\chi(M)=-16 \int_{B} c_{1}^{2}$.

The following discussion extends to all dimensions but for the sake of brevity we specialize to Calabi-Yau threefolds. Let $K_{i}, i=1, \ldots, b_{2}(B)$, span the Kähler (or ample) cone of $B$ with intersection numbers $K_{i} K_{j}=$ $c_{i j}$. Moreover, let $C^{i}$ be a basis for the dual Kähler cone. We expand the

\footnotetext{
${ }^{1}$ In the $D 5$ complete intersection case $d_{1}=d_{2}=2$. One has to add a factor $\left(1+\alpha+c_{1}\right)$ in the numerator and a factor $\left(1+2 \alpha+2 c_{1}\right)$ in the denominator.
} 
canonical class of $B$ in terms of $K_{i}$ and $C^{i}$ as:

$$
K_{B}=-c_{1}=-\sum_{i} a^{i} K_{i}=-\sum a_{i} C^{i},
$$

with $a_{i}$ and $a^{i}$ in $\mathbb{Z}$. We denote by $\mathcal{K}_{a}, a=1, \ldots, h^{1,1}(M)$, the divisors of the total space of the elliptic fibration and distinguish between $\mathcal{K}_{e}$ the divisor dual to the elliptic fibre curve and $\mathcal{K}_{i}, i=1, \ldots, b=b_{2}(B)$, which are $\pi^{*}\left(C^{i}\right)$

$$
\begin{aligned}
\mathcal{K}_{e}^{3} & =a \int_{B} c_{1}^{2}, \\
\mathcal{K}_{e}^{2} \mathcal{K}_{i} & =a a_{i}, \\
\mathcal{K}_{e} \mathcal{K}_{i} \mathcal{K}_{j} & =a c_{i j} .
\end{aligned}
$$

Here, $a$ denotes the number of sections; see table 1 . The intersection with the second Chern class of the total space can be calculated using table 1 for the elliptic and other fibres as

$$
\begin{aligned}
& \int_{M} c_{2} J_{e}= \begin{cases}\int_{B}\left(11 c_{1}^{2}+c_{2}\right) & E_{8}, \\
2 \int_{B}\left(5 c_{1}^{2}+c_{2}\right) & E_{7}, \\
3 \int_{B}\left(3 c_{1}^{2}+c_{2}\right) & E_{6}, \\
4 \int_{B}\left(2 c_{1}^{2}+c_{2}\right) & D_{5},\end{cases} \\
& \int_{M} c_{2} J_{i}=12 a_{i} .
\end{aligned}
$$

Here, we denoted by $J_{i}$ the basis of harmonic $(1,1)$ forms dual to the $\mathcal{K}_{i}$.

Let us note two properties about the intersection numbers. These properties can be established using the properties of the toric almost Fano bases $B$ and (2.7), which follows from the construction of the elliptic fibration summarized in (2.20). To start, define the matrix

$$
C_{e}=\left(\begin{array}{cccc}
\int_{B} c_{1}^{2} & a_{1} & \ldots & a_{b} \\
a_{1} & & & \\
\vdots & & c_{i j} & \\
a_{b} & & &
\end{array}\right)
$$


then we can conclude from properties of the intersection numbers and the canonical class that

$$
\operatorname{det}\left(C_{e}\right)=0
$$

A further property concerns a decoupling limit between base and fibre in the Kähler moduli space. Generally, we can make a linear change in the basis of Mori vectors $l_{i}$, which results in corresponding linear change of the basis in dual spaces of the Kähler moduli $t^{i}$ and the divisors $D_{i}$

$$
\tilde{l}_{i}=\sum_{j} m_{i j} l_{j}, \quad \tilde{t}^{i}=\sum\left(m^{T}\right)_{i j} t^{j} .
$$

To realize a decoupling between the base and the fibre, we want to find a not necessarily integer basis change, which eliminates the couplings $\tilde{\mathcal{K}}_{e}^{2} \tilde{\mathcal{K}}_{i}$ and leaves the couplings $\tilde{\mathcal{K}}_{e} \tilde{\mathcal{K}}_{i} \tilde{\mathcal{K}}_{j}$ invariant. It follows from (2.6), (2.7) and the obvious transformation of the triple intersections that there is a unique solution

$$
m=\left(\begin{array}{cccc}
1 & \frac{a^{1}}{2} & \ldots & \frac{a^{b}}{2} \\
0 & 1 & 0 \ldots & 0 \\
\vdots & & \vdots & \\
0 & 0 & \ldots 0 & 1
\end{array}\right)
$$

such that

$$
\begin{aligned}
\tilde{\mathcal{K}}_{e}^{3} & =a\left(\int_{B} c_{1}^{2}-\frac{3}{2} a_{i} a^{i}+\frac{3}{4} c_{i j} a^{i} a^{j}\right), \\
\tilde{\mathcal{K}}_{e}^{2} \tilde{\mathcal{K}}_{i} & =0 \\
\tilde{\mathcal{K}}_{e} \tilde{\mathcal{K}}_{i} \tilde{\mathcal{K}}_{j} & =a c_{i j} .
\end{aligned}
$$

As we have seen the classical topological data of the total space of the elliptic fibration follows from simple properties of the fibre and the topology of the base. We want to extend these results in the next section to the quantum cohomology of the elliptic fibration. We focus again on the Calabi-Yau threefold case, where the instanton contributions to the quantum cohomology is richest. To actually calculate quantum cohomology we need an explicit realisation of a class of examples, which we discuss in the next subsection. 


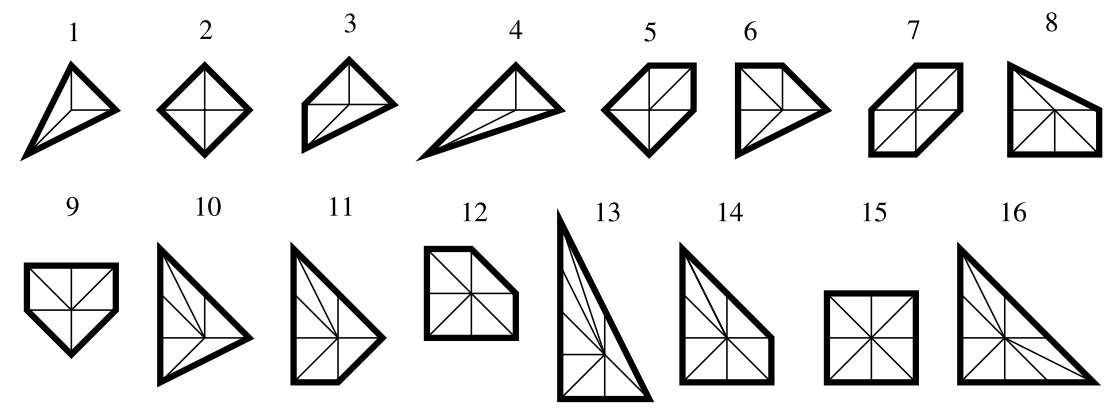

Figure 1: These are the 16 reflexive polyhedra $\Delta_{B}$ in two dimensions, which build 11 dual pairs $\left(\Delta_{B}, \Delta_{B}^{*}\right)$. Polyhedron $k$ is dual to polyhedron $17-k$ for $k=1, \ldots, 5$. The polyhedra $6, \ldots, 11$ are self-dual.

\subsection{Realizations in toric ambient spaces}

In this subsection, we discuss the toric bases $B$ leading to the above described tame elliptically fibered Calabi-Yau $d_{M}$-folds with only $I_{1}$ fibre singularities. It was observed in examples in [38], that they can be defined over toric bases defined themselves by reflexive polyhedra [6] $\Delta_{B}$ in $d_{M}-1$ dimensions. Here we explore a class of elliptic Calabi-Yau fibrations, which are defined from a reflexive polyhedra $\Delta_{B}$ as the canonical hypersurface in the toric ambient space defined by the reflexive polyhedra (2.20) following Batyrev's work [6]. Note that for each $\Delta_{B}$, one has the choice of the elliptic fibre as discussed in the previous section. We provide the toric data, including a basis for the Mori cone for this class of elliptic Calabi-Yau fibrations. The construction of the Mori cone from the star triangulation and the associated secondary fan follows the discussion in $[8,30]$. Throughout the subsection we assume some familiarity with the construction of the toric ambient spaces from polyhedra as described, e.g., in $[21,58]$.

The toric ambient spaces, which allow for smooth Calabi-Yau hypersurfaces of complex dimension $d_{M}$ as section of the canonical bundle, can be described by pairs of reflexive polyhedra $\left(\Delta, \Delta^{*}\right)$ of real dimension $d_{M}-1$. For the threefold case, the possibilities of two-dimensional reflexive base polyhedra are listed in figure 1 [6]. Together with a complete star triangulation of $\Delta$, they define a complex family of Calabi-Yau threefolds. The mirror family is given by exchanging the role of $\Delta$ and $\Delta^{*}$. A complete triangulation divides $\Delta$ in simplices of volume 1 . In a star triangulation all simplices contain the unique interior lattice point of the reflexive polyhedron. Let us give 
first two examples for toric smooth ambient spaces in which the canonical hypersurface leads to the $E_{8}$ elliptic fibration over $\mathbb{P}^{2}$ and over the Hirzebruch surface $\mathbb{F}_{1}$. The polyhedron $\Delta$ for the $E_{8}$ elliptic fibration over $\mathbb{P}^{2}$ with $\chi(M)=-540$ is given by the following data:

\begin{tabular}{llrrrr|rr}
\multicolumn{9}{c}{$\nu_{i}$} & & & $l^{(e)}$ & $l^{(1)}$ \\
$D_{0}$ & 1 & 0 & 0 & 0 & 0 & -6 & 0 \\
$D_{1}$ & 1 & 1 & 0 & -2 & -3 & 0 & 1 \\
$D_{2}$ & 1 & 0 & 1 & -2 & -3 & 0 & 1 \\
$D_{3}$ & 1 & -1 & -1 & -2 & -3 & 0 & 1 \\
$D_{z}$ & 1 & 0 & 0 & -2 & -3 & 1 & -3 \\
$D_{x}$ & 1 & 0 & 0 & 1 & 0 & 2 & 0 \\
$D_{y}$ & 1 & 0 & 0 & 0 & 1 & 3 & 0
\end{tabular}.

Here, we give the relevant points $\nu_{i}$ of the four- dimensional convex reflexive polyhedron $\Delta$ embedded into a hyperplane in a five-dimensional space and the linear relations $l^{(i)}$ spanning the Mori cone. This model has an unique star triangulation, given in (2.22). We calculate the intersection ring as follows from (2.7) with $a=1$

$$
\mathcal{R}=9 J_{\mathrm{e}}^{3}+3 J_{\mathrm{e}}^{2} J_{1}+J_{\mathrm{e}} J_{1}^{2} .
$$

The evaluation of $c_{2}$ on the basis of the Kähler cone follows from (2.8) as $\int_{M} c_{2} J_{\mathrm{e}}=102$ and $\int_{M} c_{2} J_{1}=36$.

The polyhedron $\Delta$ for the $E_{8}$ elliptic fibration over $\mathbb{F}_{1}$ with $\chi=-480$ reads

\begin{tabular}{rrrrrr|rrr|rrr} 
& \multicolumn{1}{c}{$\nu_{i}$} & \multicolumn{1}{c}{$l^{(e)}$} & $l^{(1)}$ & $l^{(2)}$ & $l^{(e)}+l^{(2)}$ & $l^{(1)}+l^{(2)}$ & $-l^{(2)}$ \\
$D_{0}$ & 1 & 0 & 0 & 0 & 0 & -6 & 0 & 0 & -6 & 0 & 0 \\
$D_{1}$ & 1 & 1 & 0 & -2 & -3 & 0 & 0 & 1 & 1 & 1 & -1 \\
$D_{2}$ & 1 & 0 & 1 & -2 & -3 & 0 & 1 & 0 & 0 & 1 & 0 \\
$D_{3}$ & 1 & -1 & -1 & -2 & -3 & 0 & 0 & 1 & 1 & 1 & -1 \\
$D_{4}$ & 1 & 0 & -1 & -2 & -3 & 0 & 1 & -1 & -1 & 0 & 1 \\
$D_{z}$ & 1 & 0 & 0 & -2 & -3 & 1 & -2 & -1 & 0 & -3 & 1 \\
$D_{x}$ & 1 & 0 & 0 & 1 & 0 & 2 & 0 & 0 & 2 & 0 & 0 \\
$D_{y}$ & 1 & 0 & 0 & 0 & 1 & 3 & 0 & 0 & 3 & 0 & 0
\end{tabular}

This example shows that there are two Calabi-Yau phases possible over $\mathbb{F}_{1}$, which are related by flopping a $\mathbb{P}^{1}$ represented by $l^{(2)}$. This transforms the 
half K3 to a del Pezzo eight surface, which can be shrunken to a point. In the first phase, the triangulation is described by (2.22) the intersection ring and $\int_{M} c_{2} J_{i}$ follows by $(2.7),(2.8)$ as

$$
\mathcal{R}=8 J_{\mathrm{e}}^{3}+3 J_{\mathrm{e}}^{2} J_{1}+J_{\mathrm{e}} J_{1}^{2}+2 J_{\mathrm{e}}^{2} J_{2}+J_{1} J_{2} J_{3} .
$$

and $\int_{M} c_{2} J_{\mathrm{e}}=92, \int_{M} c_{2} J_{1}=36$ and $\int_{M} c_{2} J_{3}=24$. For the second phase, we flop the $\mathbb{P}^{1}$ that corresponds to the Mori cone element $l^{(2)}$. Generally, if we flop the curve $C$ this changes the triple intersection of the divisors $\mathcal{K}_{i} \mathcal{K}_{j} \mathcal{K}_{k}[15]$ by

$$
\Delta_{i j k}=-\left(C \cdot \mathcal{K}_{i}\right)\left(C \cdot \mathcal{K}_{j}\right)\left(C \cdot \mathcal{K}_{k}\right)
$$

Now the intersection of the curves $C_{i}$ which correspond to the Mori cone vector $l^{(i)}$ with the toric divisors $D_{k}$ is given by $\left(C_{i} \cdot D_{k}\right)=l_{k}^{(i)}$. On the other hand, the $\mathcal{K}_{k}$ are combinations of $D_{k}$ restricted to the hypersurface so that $\left(\mathcal{K}^{k} \cdot C_{i}\right)=\delta_{i}^{k}$.

In addition, one has to change the basis in order to maintain positive intersection numbers ${ }^{2} \tilde{l}^{(e)}=l^{(e)}+l^{(2)}, \tilde{l}^{(1)}=l^{(1)}+l^{(2)}$ and $\tilde{l}^{(2)}=-l^{(2)}$. For the $(1,1)$ forms $J_{i}$, which transform dual to the curves, we then get the intersection ring in the new basis of the Kähler cone

$$
\begin{aligned}
\mathcal{R}= & 8 \tilde{J}_{\mathrm{e}}^{3}+3 \tilde{J}_{\mathrm{e}}^{2} \tilde{J}_{1}+\tilde{J}_{\mathrm{e}} \tilde{J}_{1}^{2}+9 \tilde{J}_{\mathrm{e}}^{2} \tilde{J}_{2}+3 \tilde{J}_{\mathrm{e}} \tilde{J}_{1} \tilde{J}_{2}+\tilde{J}_{1}^{2} \tilde{J}_{2}+9 \tilde{J}_{\mathrm{e}} \tilde{J}_{2}^{2} \\
& +3 \tilde{J}_{1} \tilde{J}_{2}^{2}+9 \tilde{J}_{2}^{3} .
\end{aligned}
$$

The intersections with $c_{2}$ are not affected by the flop, only the basis change has to be taken into account. In the second phase, the triangulation of the base is given in the the middle of figure 2 and the triangulation of $\Delta$ is specified by (2.21). In this phase, an $E_{8}$ del Pezzo surface can be shrunken to get to the elliptic fibration over $\mathbb{P}^{2}$. This identifies the classes of the latter example as $J_{\mathrm{e}}=\tilde{J}_{2}, J_{1}=\tilde{J}_{1}$, while the divisor dual to $\tilde{J}_{\mathrm{e}}^{3}$ is shrunken.

We denote by $\Delta_{B}$ the toric polyhedron for the base and specifying by

$$
\left\{\left(e_{1}, e_{2}\right)\right\}=\{(-2,-3),(-1,-2),(-1,-1)\}
$$

\footnotetext{
${ }^{2}$ This is one criterion that holds in a simplicial Kähler cone. The full specification is that $\int_{\mathcal{C}} J>0, \int_{\mathcal{D}} J \wedge J>0$ and $\int_{M} J \wedge J \wedge J>0$ for $J$ in the Kähler cone and $\mathcal{C}, \mathcal{D}$ curves and divisors. If the latter is simplicial and generated by $J_{i}$, then $J=$ $\sum d_{i} J_{i}$ with $d_{i}>0$.
} 


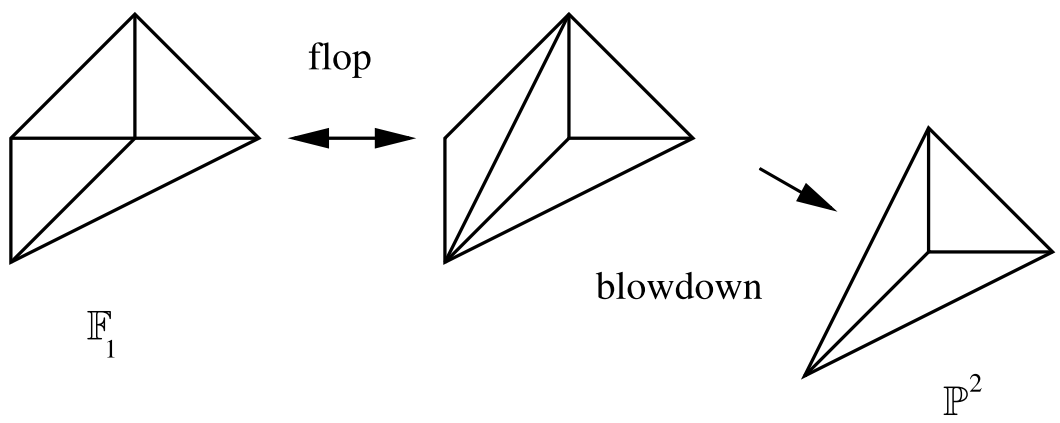

Figure 2: The base triangulation for the flop in second example and the blowdown of an $E_{8}$ del Pezzo surface.

the toric data for the $E_{8}, E_{7}, E_{6}$ fibre respectively. It is easy to see that all toric hypersurfaces with the required fibration have the following general form of the polyhedron $\Delta$ :

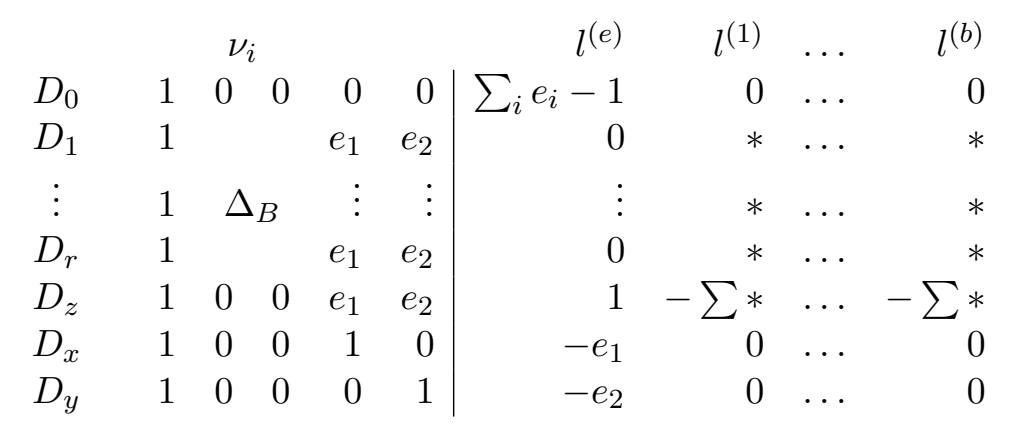

We note that the fibre elliptic curve is realized in a two-dimensional toric variety, which can be defined also by a reflexive two-dimensional polyhedron $\Delta_{F}$. It is embedded into $\Delta$, so that the inner of $\Delta_{F}$ is also the origin of $\Delta$. Its corners are given by

$$
\left\{\left(0,0, e_{1}, e_{2}\right),(0,0,1,0),(0,0,0,1)\right\} .
$$

The $E_{6}, E_{7}$ and $E_{8}$ fibre types correspond to the polyhedra in figure 1 with numbers 1,4 and 10 . To check the latter equivalence requires an change of coordinates in $S L(2, \mathbb{Z})$. The dual reflexive polyhedron $\Delta^{*}$ contains $\Delta_{F}^{*}$ embedding likewise in the coordinate plane spanned by the $3 \mathrm{rd}$ and 4 th axes. 
A triangulation of $\Delta_{B}$ as in figures 1 or 2 lifts in a universal way to a star triangulation of $\Delta$ as follows. To set the conventions denote by $\left(\nu_{i}^{B}, e_{1}, e_{2}\right)$ the points of the embedded base polyhedron $\Delta_{B}$ and label them as the points of $\Delta_{B}$ starting with the positive $x$-axis, which points to the right in the figures, and label points of $\Delta_{B}$ counter clockwise from $1, \ldots, r$. The inner point in $\Delta_{B},\left(0,0, e_{1}, e_{2}\right)$ is labelled $z$. The two remaining points of $\Delta$; $(0,0,1,0)$ and $(0,0,0,1)$ are labelled by $x$ and $y$.

Denote the $k$-th $d$-dimensional simplex in $\Delta_{B}$ by the labels of its vertices, i.e.,

$$
\operatorname{sim}_{k}^{(d)}:=\left(\lambda_{1}^{k}, \ldots, \lambda_{d+1}^{k}\right)
$$

and in particular denote the outer edges of $\Delta_{B}$ by

$$
\left\{\operatorname{ed}_{k} \mid k=1, \ldots, r\right\}:=\{(1,2), \ldots,(r, 1)\} .
$$

Any triangulation of $\Delta_{B}$ is lifted to a star triangulation of $\Delta$, which is spanned by the simplices containing besides the inner point $(0,0,0,0)$ of $\Delta$ the points with the labels

$$
\operatorname{Tr}_{\Delta}=\left\{\left(\operatorname{sim}_{k}^{(2)}, x\right),\left(\operatorname{sim}_{k}^{(2)}, y\right) \mid k=1, \ldots, p\right\} \cup\left\{\left(\operatorname{ed}_{k}, x, y\right) \mid k=1, \ldots r\right\} .
$$

In particular for star triangulations of $\Delta_{B}$, one has

$$
\operatorname{Tr}_{\Delta}=\left\{\left(\operatorname{ed}_{k}, z, x\right),\left(\operatorname{ed}_{k}, z, y\right),\left(\operatorname{ed}_{k}, x, y\right) \mid k=1, \ldots r\right\}
$$

and generators of the Mori cone for the elliptic phase contain the Mori cone generators $l^{(1)}, \ldots, l^{(b)}$, which correspond to a star triangulation of the base polyhedron, which is the one in figure 1. We list here the Mori cones of the first seven cases,

$$
\begin{array}{c|r|rr|rr|rr|rrr|}
\Delta_{B} & 1(1) & \multicolumn{2}{|c}{2(2)} & \multicolumn{2}{c}{3(2)} & \multicolumn{3}{c}{4(3)} & \multicolumn{3}{c}{5(3)} \\
\nu_{i}^{B} & l^{(1)} & l^{(1)} & l^{(2)} & l^{(1)} & l^{(2)} & l^{(1)} & l^{(2)} & l^{(1)} & l^{(2)} & l^{(3)} \\
z & -3 & -2 & -2 & -2 & -1 & 0 & -2 & -1 & -1 & -1 \\
1 & 1 & 1 & 0 & 1 & 0 & 0 & 1 & -1 & 1 & 0 \\
2 & 1 & 0 & 1 & 0 & 1 & 1 & 0 & 1 & -1 & 1 \\
3 & 1 & 1 & 0 & 1 & -1 & -2 & 1 & 0 & 1 & -1 \\
4 & & 0 & 1 & 0 & 1 & 1 & 0 & 0 & 0 & 1 \\
5 & & & & & & & & 1 & 0 & 0 \\
6 & & & & & & & & & & \\
e x & - & & & & & & & & &
\end{array}
$$




\begin{tabular}{rrr|rrrrrr|}
\multicolumn{7}{c}{$6(3)$} & \multicolumn{7}{c}{$7(4)$} \\
$l^{(1)}$ & $l^{(2)}$ & $l^{(3)}$ & $l^{(1)}$ & $l^{(2)}$ & $l^{(3)}$ & $l^{(4)}$ & $l^{(5)}$ & $l^{(6)}$ \\
-1 & -1 & 0 & -1 & -1 & -1 & -1 & -1 & -1 \\
1 & 0 & 0 & -1 & 1 & 0 & 0 & 0 & 1 \\
-1 & 1 & 0 & 1 & -1 & 1 & 0 & 0 & 0 \\
1 & -1 & 1 & 0 & 1 & -1 & 1 & 0 & 0 \\
0 & 1 & -2 & 0 & 0 & 1 & -1 & 1 & 0 \\
0 & 0 & 1 & 0 & 0 & 0 & 1 & -1 & 1 \\
& & & 1 & 0 & 0 & 0 & 1 & -1 \\
& & & & & & & &
\end{tabular}

the remaining nine cases are given in Appendix A. We indicate in the brackets behind the model the number of Kähler moduli. If the latter is smaller then the number of Mori cone generators and the dual Kähler cone are non-simplicial. This is the case for the models 7, 9 and for 11-16. In the last column, we list the number of extra triangulations. The corresponding phases involve non-star triangulations of $\Delta$ and can be reached by flops. By the rules discussed above, we can find the intersection ring and the Mori cone in phases related by flops. We understand also the blowing down of one model. Non-reflexivity possesses a slight technical difficulty in providing the data for the calculation of the instantons. The fastest way to get the data for all cases is to provide for the models 15 and 16 a simplicial Kähler cone and reach all other cases ${ }^{3}$ by flops and blowdowns. We will do this in Appendix A.

\section{Quantum geometry of elliptic fibrations}

The aim of this section is to describe the quantum geometry of elliptic fibrations. For this, we introduce the needed notions from topological string theory and discuss two examples with base $\mathbb{P}^{2}$ and $\mathbb{F}_{1}$. One of our key findings is the holomorphic anomaly (3.9). This section finishes with a discussion of the modular properties due to the monodromy group.

From the data provided in the last section, namely the Mori cone and the intersection numbers, follow differential equations as well as particular solutions, which allow us to calculate the instanton numbers as established mathematically for genus zero by Givental, Lian, Liu and Yau [24,46]. These can be calculated very efficiently using the program described in [31]. In the cases

\footnotetext{
${ }^{3}$ Except for 13 which is available on request.
} 
at hand one can evaluate the genus one data using the genus zero results, the holomorphic anomaly equation for the Ray Singer Torsion, boundary conditions provided by the evaluation of $\int_{M} J_{a} c_{2}$ and the behaviour of the discriminant at the conifold to evaluate the instantons of the elliptic fibrations.

The higher genus curves are less systematically studied on compact threefolds. However, if the total space of the elliptic fibration over a base class is a contractable rational surface, one can shrink the latter and obtain a local model on which the modular structure of higher genus amplitudes has been intensively studied. The explicit data suggest that that this structure is maintained for all classes in the base.

We summarize in the next subsection the strategy to obtain the instanton data and based on the results we propose a general form of the instantons corrected amplitude in terms of modular forms coming from the elliptic geometry of the fibre and a simple and general holomorphic anomaly formula, which governs the all genus instanton corrected amplitudes for the above discussed class of models.

In the following subsection, we use the $B$-model to prove some aspects of the proposed statements. This can establish the $A$-model results for genus 0 and 1 , since mirror symmetry is proven and the $B$-model techniques apply. Higher genus $B$-model calculations have been first extended to compact multi-moduli Calabi-Yau manifolds in [29].

\subsection{Quantum cohomology, modularity and the anomaly equations}

The basic object, the instanton corrected triple intersections $C_{a b c}\left(t^{a}\right)$ are due to special geometry all derivable from the holomorphic prepotential $F^{(0)}$, which also fixes the Kähler potential $K\left(t^{a}, \bar{t}^{a}\right)$ for the metric on the vector moduli space. At the point of maximal unipotent monodromy [13,31]

$$
\begin{aligned}
F^{(0)}\left(t^{a}\right)= & \left(X^{0}\right)^{2}\left[-\frac{t^{3}}{3 !}+A_{a b} t^{a} t^{b}+c_{a} t^{a}+\frac{\chi(M) \zeta(3)}{2(2 \pi \mathrm{i})^{3}}\right. \\
& \left.+\sum_{\gamma \in H_{2}(M, \mathbb{Z})} n_{\gamma}^{(0)} \operatorname{Li}_{3}\left(\exp \left(2 \pi \mathrm{i} \gamma_{a} t^{a}\right)\right)\right]
\end{aligned}
$$

where $t^{3}=d_{a b c} t^{a} t^{b} t^{c}$ with $d_{a b c}$ the triple intersection numbers, $c_{a}=$ $\frac{1}{24} \int_{M} c_{2} J_{a}$ and $\chi(M)$ is the Euler number of $M$. By $J_{a}, a=1, \ldots, h^{1,1}(M)$, we denote harmonic $(1,1)$ forms, which form a basis of the Kähler cone and 
the complexified Kähler parameter $t^{a}=\int_{C_{a}}(\mathrm{i} \omega+b)$, where $C_{a}$ is a curve class in the Mori cone dual to the Kähler cone and $b$ is the Neveu-Schwarz $(1,1)$-form $b$-field. The real coefficients $A_{a b}$ are not completely fixed. They are unphysical in the sense that the Kähler potential $K\left(t^{a}, \bar{t}^{a}\right)$ and $C_{a b c}\left(t^{a}\right)$ do not depend on them. The upper index (0) on the $F^{(0)}$ indicates the genus of the instanton contributions. The triple couplings receive only contributions from genus 0 . At the point of maximal unipotent monodromy, the classical topological data provide us with the $B$-model period integrals $\Pi=\left(F_{I}, X^{I}\right)=\left(\int_{B^{I}} \Omega, \int_{A_{I}} \Omega\right)^{T}$ over an integral symplectic basis of 3-cycles of the mirror geometry $\tilde{M}:\left(A_{I}, B^{I}\right), I=0, \ldots, h^{2,1}(\tilde{M})$. This is achieved by matching the $b_{3}(\tilde{M})$ solutions to the Picard-Fuchs equation with various powers of $\log \left(z_{a}\right) \sim t^{a}$, with the expected form of the $A$-model period vector

$$
\begin{aligned}
\Pi & =X^{0}\left(\begin{array}{c}
2 F^{(0)}-t^{a} \partial_{t^{a}} F^{(0)} \\
\partial_{t^{a}} F^{(0)} \\
1 \\
t^{a}
\end{array}\right) \\
& =X^{0}\left(\begin{array}{c}
\frac{t^{3}}{3 !}+c_{a} t^{a}-i \chi(M) \frac{\zeta(3)}{(2 \pi)^{3}}+2 f(p)-t^{a} \partial_{t^{a}} f(p) \\
-\frac{d_{a b c} t^{b} t^{c}}{2}+A_{a b} t^{b}+c_{a}+\partial_{t^{a}} f(p) \\
1 \\
t^{a}
\end{array}\right),
\end{aligned}
$$

where the lower case indices run from $a=1, \ldots, h^{1,1}(M)$. In the following we will set $X^{0}=1$.

One can define a generating function for the free energy in terms of a genus expansion in the coupling $g_{s}$

$$
F\left(g_{s}, t^{a}\right)=\sum_{g=0}^{\infty} g_{s}^{2 g-2} F^{(g)}\left(t^{a}\right),
$$

where the upper index $F^{(g)}\left(t^{a}\right)$ indicates as before the genus.

According to the split of the homology $H_{2}(M, \mathbb{Z})$ into the base and the fibre homology, we define $p^{\beta}=\prod_{k=1}^{b_{2}(B)} \exp \left(2 \pi \mathrm{i} \int_{\beta} \mathrm{i} \omega+b\right)=\exp \left(2 \pi \mathrm{i} \sum_{i=1}^{b_{2}(B)}\right.$ $\left.\beta_{i} t^{i}\right)$, where $\beta \in H_{2}(M, \mathbb{Z})$ lies in the image of the map $\sigma_{*}: H_{2}(B, \mathbb{Z}) \hookrightarrow$ $H_{2}(M, \mathbb{Z})$ induced by the embedding $\sigma: B \hookrightarrow M$; and we define $q=$ $\exp \left(2 \pi \mathrm{i} \int_{e} \mathrm{i} \omega+b\right)=\exp (2 \pi \mathrm{i} \tau)$, where $e \in H_{2}(M, \mathbb{Z})$ is the curve representing 
the fibre. Now we define the following objects

$$
F_{\beta}^{(g)}(\tau)=\operatorname{Coeff}\left(F^{(g)}\left(t^{a}\right), p^{\beta}\right) .
$$

We have the following universal sectors:

$$
F_{0}^{(0)}(\tau)=\left(\int_{B} c_{1}^{2}\right) \frac{t^{3}}{3 !}+\chi(M) \frac{\zeta(3)}{2(2 \pi i)^{3}}-\chi(M) \sum_{n=1}^{\infty} \operatorname{Li}_{3}\left(q^{n}\right),
$$

$$
F_{0}^{(1)}(\tau)=\left(\frac{\int_{B} c_{2}}{24}\right) \operatorname{Li}_{1}(q), \quad F_{0}^{(g>1)}(\tau)=(-1)^{g} \frac{\chi(M)}{2} \frac{\left|B_{2 g} B_{2 g-2}\right|}{2 g(2 g-2)(2 g-2) !} .
$$

We note that it follows from the expression for $F_{0}^{(0)}(q)$ that

$$
C_{\tau \tau \tau}=\int_{B} c_{1}^{2}+\frac{\chi(M)}{2} \zeta(-3)-\frac{\chi(M)}{2} \zeta(-3) E_{4}(\tau) .
$$

The $F_{\beta}^{(g)}(\tau)$ have distinguished modular properties. The $F_{\beta}^{(g)}(\tau)$ can be written in the following general form $[32,40]$ :

$$
F_{\beta}^{(g)}(\tau)=\left(\frac{q^{\frac{1}{24}}}{\eta(\tau)}\right)^{12 \sum_{i} a_{i} \beta^{i}} P_{2 g+6 \sum_{i} a_{i} \beta^{i}-2}\left(E_{2}(\tau), E_{4}(\tau), E_{6}(\tau)\right),
$$

where $\eta(\tau)$ is the Dedekind eta function (C.1), and $P_{2 g+6 \sum_{i} a_{i} \beta^{i}-2}\left(E_{2}, E_{4}\right.$, $\left.E_{6}\right)$ are (quasi)-modular forms [36] of weight $2 g+6 \sum_{i} a_{i} \beta^{i}-2$, where $E_{2 k}(\tau)$ are the Eisenstein series of weight $2 k$ (C.2). The functions $P_{m}$ are quasi-modular forms since, besides being a function of the true modular forms $E_{4}$ and $E_{6}$, they are also a function of $E_{2}$ that does not transform as a true modular form.

For the sectors $\beta>0$, which describe non-trivial dependence on the Kähler class of the base, the $E_{2}$ dependence satisfies the following recursive condition

$$
\begin{aligned}
\frac{\partial F_{\beta}^{(g)}(\tau)}{\partial E_{2}}= & \frac{1}{24} \sum_{h=0}^{g} \sum_{\beta^{\prime}+\beta^{\prime \prime}=\beta}\left(\beta^{\prime} \cdot \beta^{\prime \prime}\right) F_{\beta^{\prime}}^{(h)}(\tau) F_{\beta^{\prime \prime}}^{(g-h)}(\tau) \\
& +\frac{1}{24} \beta \cdot\left(\beta-K_{B}\right) F_{\beta}^{(g-1)}(\tau) .
\end{aligned}
$$

We derive the above relations in Section 4. For the other types of elliptic fibrations $E_{7}, E_{6}, \& D_{5}$, the right-hand side is divided by $a=2,3,4$, 
respectively. Equation (3.9) generalizes the similar Equation (1.2) in [32], to arbitrary classes in the base and types of fibres. In particular, if one restricts to elliptic fibrations over the blow up of $\mathbb{P}^{2}$ and the Hirzebruch surface $\mathbb{F}_{1}$ the rational fibre class in the base (3.9) becomes the Equation of [32] counting curves of higher genus on the $E_{8}, E_{7}, E_{6}, \& D_{5}$ del Pezzo surfaces. The form (3.8) and its relation to [32] has been observed in [40] for the Hirzebruch surface $\mathbb{F}_{0}$ as base. A derivation of Equation (3.9) is given in Section 4.

\subsection{The $B$-model approach to elliptically fibred Calabi-Yau spaces}

We continue the discussion with some $B$-model aspects of elliptically fibred Calabi-Yau manifolds. We assume some familiarity with the formalism developed in $[30,31]$ and concentrate on features relevant and common to the $B$-model geometry of elliptic fibrations and how they emerge from the topological data of the $A$-model discussed in Section 2.

The vectors $l^{(i)}$ are the generators of the Mori cone, i.e., the cone dual to the Kähler cone. As such they reflect classical properties of the Kähler moduli space and the classical intersection numbers, like the Euler number and the evaluation of $\int_{M} c_{2} \omega_{a}$ on the basis of Kähler forms on the elliptic fibration.

On the other hand, the differential operators

$$
\left(\prod_{l_{i}^{(r)}>0} \partial_{a_{i}}^{l_{i}^{(r)}}-\prod_{l_{i}^{(r)}<0} \partial_{a_{i}}^{-l_{i}^{(r)}}\right) \tilde{\Pi}=0,
$$

annihilate the periods $\tilde{\Pi}=\frac{1}{a_{0}} \Pi$ of the mirror $\tilde{M}$. Here, the $a_{i}$ are the coefficients of the monomials in the equation defining $\tilde{M}$. They are related to the natural large complex structure variables of $\tilde{M}$ by

$$
z_{r}=(-1)^{l_{0}^{r}} \prod_{i} a_{i}^{l_{i}^{r}}
$$

Note that $\Pi$ is well defined on $\tilde{M}$, while $\tilde{\Pi}$ is not an invariant definition of periods on $\tilde{M}$. However by commuting out $a_{0}^{-1}$ one can rewrite Equations (3.10) so that they annihilate $\Pi$. Further, they can be expressed in the independent complex variables $z_{r}$ using the gauge condition $\theta_{a_{i}}=\sum_{r} l_{i}^{k} \theta_{z_{r}}$, where $\theta_{x}=x \frac{d}{d x}$ denotes the logarithmic derivative. Equations (3.10) reflect symmetries of the holomorphic $(3,0)$ form and every positive $l$ in the Mori cone (3.10) leads to a differential operator annihilating $\Pi$. The operators 
obtained in this way are contained in the left differential ideal annihilating $\Pi$, but they do not generate this ideal. There is however a factorization procedure, basically factoring polynomials $P(\theta)$ to the left, that leads in our examples to a finite set of generators that determines linear combinations of periods as their solutions. It is referred to as a complete set of Picard-Fuchs operators. In this way, properties of the instanton corrected moduli space of $M$, often called the quantum Kähler moduli space are intimately related to the $l^{(r)}$ and below we will relate some of it properties to the topology of $M$.

In particular, the Mori generator $l^{(e)}$ determines to a large extend the geometry of the elliptic fibre modulus. As one sees from $(2.20)$ the mixing between the base and the fibre is encoded in the $z$ row of $l^{(i)}$, $i=1, \ldots, h^{1,1}(B)$ and $l^{(e)}$. Let us call this the $z$-component of $l^{(i)}$ and the corresponding variable $a_{z}$.

Following the procedure described above, one obtains after factorizing from $l^{(e)}$ a second-order generator Picard-Fuchs operator. For the fibrations types introduced before it is given by

$$
\mathcal{L}_{\mathrm{e}}^{k}=\theta_{\mathrm{e}}\left(\theta_{\mathrm{e}}-\sum_{i} a^{i} \theta_{i}\right)-\mathcal{D}^{K},
$$

where $k=E 8, E 7, E 6, D 5$ refers to the fibration type and $\mathcal{D}^{K}$ contains the dependence on the type

$$
\begin{aligned}
& \mathcal{D}^{E 8}=12\left(6 \theta_{\mathrm{e}}-1\right)\left(6 \theta_{\mathrm{e}}-5\right) z_{\mathrm{e}}, \quad \mathcal{D}^{E 7}=4\left(4 \theta_{\mathrm{e}}-1\right)\left(4 \theta_{\mathrm{e}}-3\right) z_{\mathrm{e}} \\
& \mathcal{D}^{E 6}=3\left(3 \theta_{\mathrm{e}}-1\right)\left(3 \theta_{\mathrm{e}}-2\right) z_{\mathrm{e}}, \quad \mathcal{D}^{D 5}=4\left(2 \theta_{\mathrm{e}}-1\right)^{2} z_{\mathrm{e}}
\end{aligned}
$$

Formally setting $\theta_{i}=0$ corresponds to the large base limit. Then Equation (3.12) becomes the Picard-Fuchs operator, which annihilates the periods over the standard holomorphic differential on the corresponding family of elliptic curves.

In the limit of large fibre one gets as local model the total space of the canonical line bundle $\mathcal{O}\left(K_{B}\right) \rightarrow B$ over the Fano base $B$. Local mirror symmetry associates to such non-compact Calabi-Yau manifolds a genus one curve with a meromorphic 1 -form $\lambda$ that is the limit of the holomorphic $(3,0)$-form. The local Picard-Fuchs system $\mathcal{L}_{i}^{B}$ annihilating the periods $\Pi_{l o c}$ of $\lambda$ can be obtained as a limit of the compact Picard-Fuchs system for $l^{(i)}, i=1, \ldots, h^{1,1}(B)$ by formally setting $\theta_{\mathrm{e}}=0$. It follows directly from (3.10), since the Mori generators of the base have vanishing first entry and commuting out $a_{0}^{-1}$ becomes trivial. Differently then for the elliptic curve of the fibre, these Picard-Fuchs operators do not annihilate the periods over 
holomorphic differential one form of the elliptic curve, which are $\frac{1}{a_{z}} \Pi_{\text {loc }}$. Given the local Picard-Fuchs system the dependence on $\theta_{\mathrm{e}}$ can be restored by replacing $\theta_{a_{z}}$ by $\theta_{\mathrm{e}}-\sum_{i} a_{i} \theta_{i}$ instead of $-\sum_{i} a_{i} \theta_{i}$. Since $l^{(i)}$ is negative $\theta_{\mathrm{e}}$ appears in $\mathcal{L}_{b}^{i}$ only multiplied by at least one explicit $z_{i}^{b}$ factor.

There are important conclusions that follow already from the general form of the Picard-Fuchs system. To see them it is convenient to rescale $x_{\mathrm{e}}=c_{k} z_{\mathrm{e}}$, where $c_{E 8}=432, c_{E 7}=64, c_{E 6}=27, c_{D 5}=16$. It is often useful to also rescale the $z_{i}$ in a similar fashion and call them $x_{i}$.

The effect of this is that the symbols of the Picard-Fuchs system become the same for all fibre types. From this, we can conclude that for all fibre types the Yukawa-couplings and the discriminants are identical in the rescaled variables.

The second conclusion is that the Picard-Fuchs equation of the compact Calabi-Yau is invariant under the $\mathbb{Z}_{2}$ variable transformation

$$
x_{\mathrm{e}} \rightarrow\left(1-x_{\mathrm{e}}\right), \quad x_{i} \rightarrow\left(-\frac{x_{\mathrm{e}}}{1-x_{\mathrm{e}}}\right)^{a^{i}} x_{i}
$$

This means that there is always a $\mathbb{Z}_{2}$ involution acting on the moduli space parameterized by $\left(x_{\mathrm{e}}, x_{i}\right)$, which must be divided out to obtain the truly independent values of the parameters.

Another consequence of this statement is that the discriminants $\Delta_{i}\left(x_{j}\right)$ of the base Picard-Fuchs system determine the discriminant locus of the global system apart from the fibre related $\Delta\left(x_{\mathrm{e}}\right)$ components. The former contains always a conifold component $\Delta_{c}\left(x_{j}\right)$ and only that one, if there are no points on the edges of the $2 \mathrm{~d}$ polyhedron. Points on the edges correspond to $S U(2)$ or $S U(3)$ gauge symmetry enhancement discriminants, which contain only $x_{i}$ variables dual to Kähler classes, whose $a^{i}=0$. They are therefore invariant under (3.14). Moreover the lowest order term in the conifold discriminant is a constant and highest terms are weighted monomials of degree $\chi(B)$ with weights for the $x_{i} a^{i}$ or 1 if $a^{i}=0$. It follows by (3.14) that the transformed conifold discriminant $\Delta_{c}^{\prime}\left(x_{j}\right) \sim\left(1-x_{\mathrm{e}}\right)^{\chi(B)}+\mathcal{O}\left(x_{i}\right)$.

3.2.1. Examples: elliptic fibrations over $\mathbb{P}^{2}$ and $\mathbb{F}_{1}$. Let us demonstrate the above general statements with a couple of examples. We discuss the $E 8$ elliptic fibration with base $\mathbb{P}^{2}$ and with base $\mathbb{F}_{1}$.

For the first example, the Mori vectors are given as

$$
\begin{aligned}
& l^{(e)}=(-6,3,2,1,0,0,0), \\
& l^{(1)}=(0,0,0,-3,1,1,1) .
\end{aligned}
$$


Form this, we can derive the following set of Picard-Fuchs equations, where we denote $\theta_{i}=z_{i} \partial_{z_{i}}$.

$$
\begin{aligned}
& \mathcal{L}_{\mathrm{e}}=\theta_{\mathrm{e}}\left(\theta_{\mathrm{e}}-3 \theta_{2}\right)-12 z_{\mathrm{e}}\left(6 \theta_{\mathrm{e}}+5\right)\left(6 \theta_{\mathrm{e}}+1\right), \\
& \mathcal{L}_{1}=\theta_{2}^{3}-z_{2}\left(\theta_{\mathrm{e}}-3 \theta_{2}\right)\left(\theta_{\mathrm{e}}-3 \theta_{2}-1\right)\left(\theta_{\mathrm{e}}-3 \theta_{2}-2\right) .
\end{aligned}
$$

The Yukawa couplings for this example read as follows, where we use $z_{\mathrm{e}}=$ $\frac{x_{\mathrm{e}}}{432}, z_{1}=\frac{x_{1}}{27}$ and the discriminants $\Delta_{\mathrm{e}}=1-3 x_{\mathrm{e}}+3 x_{\mathrm{e}}^{2}-x_{\mathrm{e}}^{3}-x_{\mathrm{e}}^{3} x_{1}$ and $\Delta_{1}=1+x_{1}$

$$
\begin{aligned}
C_{e e e} & =\frac{9}{x_{\mathrm{e}}^{3} \Delta_{\mathrm{e}}}, \\
C_{e e 1} & =-\frac{3\left(-1+x_{\mathrm{e}}\right)}{x_{\mathrm{e}}^{2} x_{1} \Delta_{1}}, \\
C_{e 11} & =\frac{\left(-1+x_{\mathrm{e}}\right)^{2}}{x_{\mathrm{e}} x_{1}^{2} \Delta_{\mathrm{e}}}, \\
C_{111} & =\frac{1-3 x_{\mathrm{e}}+3 x_{\mathrm{e}}^{2}}{3 x_{1}^{2} \Delta_{\mathrm{e}} \Delta_{1}} .
\end{aligned}
$$

The second example over $\mathbb{F}_{1}$ has the following three generators of the Mori cone

$$
\begin{aligned}
& l^{(e)}=\left(\begin{array}{lllllll}
-6,3, & 2, & 1, & 0, & 0, & 0, & 0
\end{array}\right), \\
& l^{(1)}=(0,0,0,-1,-1,0, \quad 1, \quad 1) \text {, } \\
& l^{(2)}=(0,0,0,-2, \quad 1, \quad 1,0,0) \text {, }
\end{aligned}
$$

and gives rise to the following Picard-Fuchs equations:

$$
\begin{aligned}
& \mathcal{L}_{\mathrm{e}}=\theta_{\mathrm{e}}\left(\theta_{\mathrm{e}}-2 \theta_{2}-\theta_{1}\right)-12 z_{\mathrm{e}}\left(6 \theta_{\mathrm{e}}+5\right)\left(6 \theta_{\mathrm{e}}+1\right), \\
& \mathcal{L}_{1}=\theta_{1}^{2}-z_{1}\left(\theta_{1}-\theta_{2}\right)\left(2 \theta_{2}+\theta_{1}-\theta_{\mathrm{e}}\right), \\
& \mathcal{L}_{2}=\theta_{2}\left(\theta_{2}-\theta_{1}\right)-z_{2}\left(2 \theta_{2}+\theta_{1}-\theta_{\mathrm{e}}\right)\left(2 \theta_{2}+\theta_{1}-\theta_{\mathrm{e}}+1\right) .
\end{aligned}
$$

This example contains the rational elliptic surface, which we discuss in detail in Section 6. Furthermore, we focus on this example to give a proof of the holomorphic anomaly at genus zero by using mirror symmetry in Section 4.1.

\subsection{Modular subgroup of monodromy group}

The deeper origin of the appearance of modular forms is the monodromy group of the Calabi-Yau threefold. Candelas et al. [14] explain that in the large volume limit of $X_{18}[1,1,1,6,9]$, which corresponds to the elliptic 


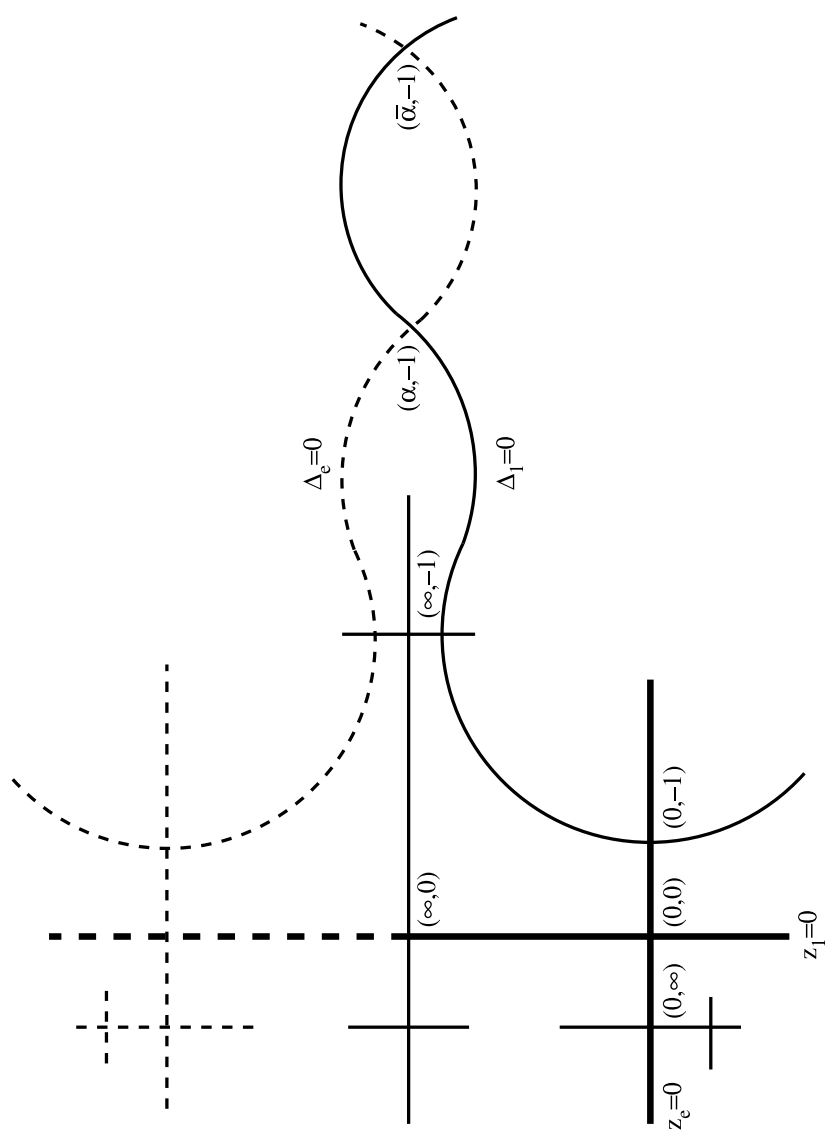

Figure 3: The moduli space for the elliptic Calabi-Yau fibration over $\mathbb{P}^{2}$ $X_{18}[1,1,1,6,9]$.

fibration over $\mathbb{P}^{2}$, the monodromy group reduces to an $S L(2, \mathbb{Z})$ monodromy group. This section recalls the appearance of this modular group and how it generalizes to other elliptic fibrations. The moduli space of $X_{18}[1,1,1,6,9]$ with the degeneration loci is portrayed in figure 3 .

We continue by recalling the monodromy for the model in [14] adapted to our discussion. The fundamental solution is given by

$$
\begin{aligned}
w_{0}\left(z_{\mathrm{e}}, z_{1}\right) & =\sum_{m, n=0}^{\infty} \frac{(18 n+6 m) !}{(9 n+3 m) !(6 n+2 m) !(n !)^{3} m !} z_{\mathrm{e}}^{3 n+m} z_{1}^{m} \\
& =\sum_{k=0}^{\infty} \frac{(6 k) !}{k !(2 k) !(3 k) !} z_{\mathrm{e}}^{k} U_{k}\left(z_{1}\right)
\end{aligned}
$$


with

$$
\begin{aligned}
U_{\nu}\left(z_{1}\right) & =z_{1}^{\nu} \sum_{n=0}^{\infty} \frac{\nu !}{(n !)^{3} \Gamma(\nu-3 n+1)} z_{1}^{-3 n} \\
& =z_{1}^{\nu} \sum_{n=0}^{\infty} \frac{\Gamma(3 n-\nu)}{\Gamma(-\nu)(n !)^{3}} z_{1}^{-3 n},
\end{aligned}
$$

which is a finite polynomial for positive integers $\nu$, since $\Gamma(\nu-3 n+1)=\infty$ for sufficiently large $n$. The translation to the parameters in [14] is $\left(z_{\mathrm{e}}, z_{1}\right)=$ $\left((18 \psi)^{-6},-3 \phi\right)$. The natural coordinates obtained from toric methods are $\tilde{z}_{\mathrm{e}}=z_{\mathrm{e}} z_{1}$ and $\tilde{z}_{1}=z_{1}^{-3}$. Note that the second line (3.20) makes manifest the presence of the elliptic curve in the geometry. For this regime of the parameters one can easily find logarithmic solutions by taking derivatives to $k$ and $n[30]$

$$
\begin{aligned}
& 2 \pi \mathrm{i} w_{e}^{(1)}\left(z_{\mathrm{e}}, z_{1}\right)=\log \left(z_{\mathrm{e}} z_{1}\right) w_{0}+\cdots, \\
& 2 \pi \mathrm{i} w_{1}^{(1)}\left(z_{\mathrm{e}}, z_{1}\right)=-3 \log \left(z_{1}\right) w_{0}+\cdots .
\end{aligned}
$$

The periods are defined by $\tau=w_{\mathrm{e}}^{(1)} / w_{0}$ and $t_{1}=w_{1}^{(1)} / w_{0}$ and $q=\mathrm{e}^{2 \pi \mathrm{i} \tau}$, $q_{1}=\mathrm{e}^{2 \pi \mathrm{i} t_{1}}$.

The two monodromies which generate the modular group are

$$
\begin{aligned}
& M_{0}: \quad\left(z_{\mathrm{e}}, z_{1}\right) \rightarrow\left(\mathrm{e}^{2 \pi \mathrm{i}} z_{\mathrm{e}}, z_{1}\right), \quad z_{\mathrm{e}} \text { small, } \quad z_{1} \text { large } \\
& M_{\infty}: \quad\left(z_{\mathrm{e}}, z_{1}\right) \rightarrow\left(\mathrm{e}^{2 \pi \mathrm{i}} z_{\mathrm{e}}, z_{1}\right), \quad z_{\mathrm{e}} \text { large, } \quad z_{1} \text { large }
\end{aligned}
$$

The monodromy around $z_{\mathrm{e}}=0$ follows directly from (3.22), it acts as

$$
\mathbf{M}_{0}=\left(\begin{array}{ll}
1 & 1 \\
0 & 1
\end{array}\right)
$$

on $\left(w_{\mathrm{e}}^{(1)}, w_{0}\right)^{\mathrm{T}}$. To determine the action on the periods of $M_{\infty}$, we need to analytically continue $w_{0}$ and $w_{1}^{(1)}$ to large $z_{\mathrm{e}}$. To this end, we write $w_{0}$ as a Barnes integral

$$
w_{0}\left(z_{\mathrm{e}}, z_{1}\right)=\frac{1}{2 \pi \mathrm{i}} \int_{C} d s \frac{\Gamma(-s) \Gamma(6 s+1)}{\Gamma(2 s+1) \Gamma(3 s+1)} \mathrm{e}^{\pi \mathrm{i} s} z_{\mathrm{e}}^{s} U_{s}\left(z_{1}\right),
$$

where $C$ is the vertical line from $-\mathrm{i} \infty-\varepsilon$ to $\mathrm{i} \infty-\varepsilon$. For small $\left|z_{\mathrm{e}}\right|$ the contour can be deformed to the right giving back the expression in (3.20). 
For large $\left|z_{\mathrm{e}}\right|$ one instead obtains the expansion

$$
w_{0}\left(z_{\mathrm{e}}, z_{1}\right)=\frac{1}{6 \pi^{2}} \sum_{r=1,5} \sin (\pi r / 3) \sum_{k=0}^{\infty} a_{r}(k)\left(-z_{\mathrm{e}}\right)^{-k-\frac{r}{6}} U_{-k-r / 6}\left(z_{1}\right)
$$

with

$$
a_{r}(k)=(-1)^{k} \frac{\Gamma(k+r / 6) \Gamma(2 k+r / 3) \Gamma(3 k+r / 2)}{\Gamma(6 k+r)} .
$$

The logarithmic solution $w_{\mathrm{e}}^{(1)}$ is given similarly by

$$
\begin{aligned}
w_{\mathrm{e}}^{(1)}\left(z_{\mathrm{e}}, z_{1}\right) & =\frac{1}{2 \pi \mathrm{i}} \int_{C} d s \frac{\Gamma(-s)^{2} \Gamma(6 s+1) \Gamma(s+1)}{\Gamma(2 s+1) \Gamma(3 s+1)} \mathrm{e}^{2 \pi \mathrm{i} s} z_{\mathrm{e}}^{s} U_{s}\left(z_{1}\right), \\
& =\frac{1}{6 \pi^{2} \mathrm{i}} \sum_{r=1,5} \mathrm{e}^{-\pi \mathrm{i} r / 6} \cos (\pi r / 6) \sum_{k=0}^{\infty} a_{r}(k)\left(-z_{\mathrm{e}}\right)^{-k-\frac{r}{6}} U_{-k-r / 6}\left(z_{1}\right) .
\end{aligned}
$$

To determine the action of $M_{\infty}$, we define the basis $f_{r}\left(z_{\mathrm{e}}, z_{1}\right)=\sum_{n=0}^{\infty}$ $a_{r}(k)\left(-z_{\mathrm{e}}\right)^{-k-\frac{r}{6}} U_{-k-r / 6}\left(z_{1}\right)$ for $r=1,5$, and the matrix $\mathbf{A}$ that relates the two bases: $\left(w_{\mathrm{e}}^{(1)}, w_{1}^{(1)}\right)^{\mathrm{T}}=\mathbf{A}\left(f_{1}, f_{5}\right)^{\mathrm{T}}$. Clearly, $M_{\infty}$ acts diagonally on the $f_{r}: \mathbf{T}=\operatorname{diag}\left(\alpha^{-1}, \alpha^{-5}\right)$ with $\alpha=\mathrm{e}^{2 \pi \mathrm{i} / 6}$, which gives for $\mathbf{M}_{\infty}$

$$
\mathbf{M}_{\infty}=\mathbf{A T A}^{-1}=\left(\begin{array}{cc}
0 & -1 \\
1 & 1
\end{array}\right) \in S L_{2}(\mathbb{Z}) .
$$

This gives for the monodromy around the conifold locus

$$
\mathbf{M}_{1}=\mathbf{M}_{0} \mathbf{M}_{\infty}^{-1}=\left(\begin{array}{ll}
1 & 0 \\
1 & 1
\end{array}\right)
$$

The generator $S=\left(\begin{array}{cc}0 & 1 \\ -1 & 0\end{array}\right)$ of $S L_{2}(\mathbb{Z})$ corresponds to $\mathbf{M}_{0} \mathbf{M}_{\infty}^{-1}$.

The large volume limit is such that $u=q^{3 / 2} p \rightarrow 0$. We see that $M_{0}$ and $M_{\infty}$ map small $u$ to small $u$. The monodromies act on $u$ by [14]

$$
M_{0} u=-u, \quad M_{\infty} u=u
$$

Thus we have established an action of $S L_{2}(\mathbb{Z})$ on the boundary of the moduli space.

The above analysis can be extended straightforwardly to the other types of fibrations using the expansions (B.2). The matrix $\mathbf{M}_{0}$ is for all fibre types the same. We find that $\mathbf{M}_{\infty}=\left(\begin{array}{cc}1-a-1 \\ a & 1\end{array}\right) \in \Gamma_{0}(a)$ for $a=2,3$ and 4 
corresponding to the fibre types $E_{7}, E_{6}$ and $D_{5}$. Note that $\mathbf{M}_{\infty}$ has order 4 and 3 for $a=2$ and 3, respectively, while the order is infinite for $a=4$. Generalization to other base surfaces $B$ is also straightforward. In case of multiple 2-cycles in the base, it is natural to define parameters for each base class: $u_{i}=q^{a_{i} / 2} p_{i}$, with $p_{i}=\exp \left(2 \pi \mathrm{i} t^{i}\right), i=1, \ldots, b_{2}(B)$. This is precisely the change of parameters given by (2.12). These transform under the action of $M_{0}$ and $M_{\infty}$ as:

$$
M_{0} u_{i}=(-)^{a_{i}} u_{i}, \quad M_{\infty} u_{i}=u_{i}
$$

\section{Derivation of the holomorphic anomaly equation}

\subsection{The elliptic fibration over $\mathbb{F}_{1}$}

In this section, we discuss the holomorphic anomaly equation that arises for elliptically fibered Calabi-Yau threefolds. By using mirror symmetry the anomaly for genus 0 is proven for the base being $\mathbb{F}_{1}$. We further establish a derivation from the BCOV holomorphic anomaly equations.

We start by deriving the holomorphic anomaly equation at genus zero by adapting the proof which appeared in [32] for a similar geometry. For this, we determine the solution to the Picard-Fuchs equation and express the solution in terms of modular forms. After determining the mirror map, we find a recursive relation in the functions $c_{m}\left(x_{\mathrm{e}}\right)$, which are related to the genus 0 topological amplitude. This can be used to prove the holomorphic anomaly equation for genus 0. Furthermore, the genus 0 topological amplitude can be expressed in terms of standard Eisenstein series of the elliptic parameter. We start by studying the Picard-Fuchs operator associated to the elliptic fiber $X_{6}[1,2,3]$ only. Denoting by $\theta_{\mathrm{e}}=z_{\mathrm{e}} \partial_{z_{\mathrm{e}}}$ the Picard-Fuchs operator can be written as

$$
\mathcal{L}=\theta_{\mathrm{e}}^{2}-12 z_{\mathrm{e}}\left(6 \theta_{\mathrm{e}}+5\right)\left(6 \theta_{\mathrm{e}}+1\right) .
$$

One can immediately write down two solutions as power series expansions around $z_{\mathrm{e}}=0$. They are given by

$$
\phi\left(z_{\mathrm{e}}\right)=\sum_{n \geq 0} a_{n} z_{\mathrm{e}}^{n}, \quad \tilde{\phi}\left(z_{\mathrm{e}}\right)=\log \left(z_{\mathrm{e}}\right) \phi\left(z_{\mathrm{e}}\right)+\sum_{n \geq 0} b_{n} z_{\mathrm{e}}^{n}
$$


with

$$
\begin{aligned}
& a_{n}=\frac{(6 n) !}{(3 n) !(2 n) ! n !}, \\
& b_{n}=a_{n}(6 \psi(1+6 n)-3 \psi(1+3 n)-2 \psi(1+2 n)-\psi(1+n)),
\end{aligned}
$$

where $\psi(z)$ denotes the digamma function. The mirror map is thus given by

$$
2 \pi \mathrm{i} \tau=\frac{\tilde{\phi}\left(z_{\mathrm{e}}\right)}{\phi\left(z_{\mathrm{e}}\right)}
$$

Using standard techniques from the Gauss-Schwarz theory for the PicardFuchs equation (cf. [45]) one observes

$$
j(\tau)=\frac{1}{z_{\mathrm{e}}\left(1-432 z_{\mathrm{e}}\right)},
$$

which can be inverted to yield

$$
z_{\mathrm{e}}(\tau)=\frac{1}{864}(1-\sqrt{1-1728 / j(\tau)})=q-312 q^{2}+\mathcal{O}\left(q^{3}\right) .
$$

Further, the polynomial solution $\phi\left(x_{\mathrm{e}}\right)$ can be expressed in terms of modular forms as

$$
\phi\left(z_{\mathrm{e}}\right)={ }_{2} F_{1}\left(\frac{5}{6}, \frac{1}{6}, 1 ; 432 z_{\mathrm{e}}\right)=\sqrt[4]{E_{4}(\tau)}
$$

from which one can conclude that

$$
\begin{aligned}
E_{4}(\tau) & =\phi^{4}\left(z_{\mathrm{e}}\right) \\
E_{6}(\tau) & =\phi^{6}\left(z_{\mathrm{e}}\right)\left(1-864 z_{\mathrm{e}}\right), \\
\Delta(\tau) & =\phi^{12}\left(z_{\mathrm{e}}\right) z_{\mathrm{e}}\left(1-432 z_{\mathrm{e}}\right), \\
\frac{1}{2 \pi \mathrm{i}} \frac{d z_{\mathrm{e}}}{d \tau} & =\phi^{2}\left(z_{\mathrm{e}}\right) z_{\mathrm{e}}\left(1-432 z_{\mathrm{e}}\right) .
\end{aligned}
$$

Let us now examine the periods of the mirror geometry $\tilde{M}$ in the limit that the fibre $f$ over the Hirzebruch surface $\mathbb{F}_{1}$ becomes small. At $z_{\mathrm{e}}=$ $z_{1}=z_{2}$ we have a point of maximal unipotent monodromy. In particular, this implies that one has among the eight periods one holomorphic one, $w_{0}\left(z_{\mathrm{e}}, z_{1}, z_{2}\right)$, one that starts with $\log \left(z_{\mathrm{e}}\right), w_{\mathrm{e}}^{(1)}\left(z_{\mathrm{e}}, z_{1}, z_{2}\right)$, and one that starts with $\log \left(z_{1}\right), w_{1}^{(1)}\left(z_{\mathrm{e}}, z_{1}, z_{2}\right)$. In the limit of small fibre $f$, i.e., $z_{2}=0$, one 
obtains for these periods [32]:

$$
\begin{aligned}
w_{0}\left(z_{\mathrm{e}}, z_{1}, 0\right) & =\phi\left(z_{\mathrm{e}}\right) \\
w_{\mathrm{e}}^{(1)}\left(z_{\mathrm{e}}, z_{1}, 0\right) & =\tilde{\phi}\left(z_{\mathrm{e}}\right) \\
w_{1}^{(1)}\left(z_{\mathrm{e}}, z_{1}, 0\right) & =\log \left(z_{1}\right) \phi\left(z_{\mathrm{e}}\right)+\xi\left(z_{\mathrm{e}}\right)+\sum_{m \geq 1}\left(\mathcal{L}_{m} \phi\left(z_{\mathrm{e}}\right)\right) z_{1}^{m}
\end{aligned}
$$

with

$$
\xi\left(z_{\mathrm{e}}\right)=\sum_{n \geq 0} a_{n}(\psi(1+n)-\psi(1)) z_{\mathrm{e}}^{n}
$$

and

$$
\mathcal{L}_{m}=\frac{(-)^{m}}{m(m !)} \prod_{k=1}^{m}\left(\theta_{z_{\mathrm{e}}}-k+1\right) .
$$

This can be obtained by applying the Frobenius method to derive the period integrals, see, e.g., [31]. The mirror map reads

$$
2 \pi \mathrm{i} t_{j}=\frac{w_{j}^{(1)}\left(z_{\mathrm{e}}, z_{1}, 0\right)}{w_{0}\left(z_{\mathrm{e}}, z_{1}, 0\right)}, \quad j=e, 1 .
$$

Comparing this with our previous discussion about the Picard-Fuchs operator of the elliptic fibre we see that for $t_{\mathrm{e}}=\tau$ there is nothing left to discuss. Hence, let us study the mirror map associated to $t_{1}=t$. We observe that by formally inverting, the inverse mirror map can be determined iteratively through the relation [32]

$$
z_{1}(q, p)=p \zeta e^{-\sum_{m \geq 1} c_{m}\left(z_{\mathrm{e}}\right) z_{1}^{m}},
$$

where $\zeta=\mathrm{e}^{-\frac{\xi\left(z_{\mathrm{e}}\right)}{\phi\left(z_{\mathrm{e}}\right)}}, p=\exp (2 \pi \mathrm{i} t)$ and

$$
c_{m}\left(z_{\mathrm{e}}\right)=\frac{\mathcal{L}_{m} \phi\left(z_{\mathrm{e}}\right)}{\phi\left(z_{\mathrm{e}}\right)} .
$$

Using Equation (4.8) $c_{1}\left(z_{\mathrm{e}}\right)$ is given by

$$
\begin{aligned}
c_{1}\left(z_{\mathrm{e}}\right) & =-\frac{1}{12}\left(f_{1}-2\right)-\frac{f_{1}}{12} \frac{E_{2}(\tau)}{\phi^{2}\left(z_{\mathrm{e}}\right)} \\
& =-\frac{1}{\phi^{6}} \frac{f_{1}}{12}\left(E_{2} E_{4}-E_{6}\right),
\end{aligned}
$$


where we introduced $f_{1}=\left(1-432 z_{\mathrm{e}}\right)^{-1}$. In order to obtain the other $c_{m}\left(z_{\mathrm{e}}\right)$ one uses

$$
\begin{aligned}
\theta_{\mathrm{e}} f_{1} & =f_{1}\left(f_{1}-1\right), \\
\theta_{\mathrm{e}}\left(\frac{E_{2}}{\phi^{2}}\right) & =-\frac{1}{\phi^{8}} \frac{f_{1}}{12}\left(E_{2}^{2} E_{4}-2 E_{2} E_{6}+E_{4}^{2}\right), \\
\theta_{\mathrm{e}}\left(\frac{E_{6}}{\phi^{6}}\right) & =-\frac{1}{\phi^{12}} \frac{f_{1}}{12}\left(6 E_{4}^{3}-6 E_{6}^{2}\right),
\end{aligned}
$$

and finds the following kind of structure. One can show inductively that

$$
c_{m}\left(z_{\mathrm{e}}\right)=\frac{1}{\phi^{6 m}}\left(\frac{f_{1}}{12}\right)^{m} Q_{6 m}\left(E_{2}, E_{4}, E_{6}\right),
$$

where $Q_{6 m}$ is a quasi-homogeneous polynomial of degree $6 m$ and type $(2,4,6)$, i.e.,

$$
Q_{6 m}\left(\lambda^{2} z_{\mathrm{e}}, \lambda^{4} z_{1}, \lambda^{6} z_{2}\right)=\lambda^{6 m} Q_{6 m}\left(z_{\mathrm{e}}, z_{1}, z_{2}\right) .
$$

Also by induction, it follows from (4.15) and (4.16) that $Q_{6 m}$ is linear in $E_{2}$. This allows us to write a second structure which is analogous to the one appearing in [32] and given by

$$
c_{m}\left(z_{\mathrm{e}}\right)=B_{m} \frac{E_{2}}{\phi^{2}}+D_{m}
$$

where the coefficients $B_{m}, D_{m}$ obey the following recursion relation:

$$
\begin{aligned}
& B_{m+1}=-\frac{m}{(m+1)^{2}}\left[\left(\theta_{z_{\mathrm{e}}}-m\right) B_{m}+D_{1} B_{m}-B_{1} D_{m}\right], \\
& D_{m+1}=-\frac{m}{(m+1)^{2}}\left[\left(\theta_{z_{\mathrm{e}}}-m\right) D_{m}-D_{1} D_{m}+B_{1} B_{m}\right],
\end{aligned}
$$

with $B_{1}=-\frac{f_{1}}{12}$ and $D_{1}=-\frac{1}{12}\left(f_{1}-2\right)$. A formal solution to the recursion relation (4.19) can be given by

$$
\begin{aligned}
& B_{m}=-\frac{f_{m}}{12}, \\
& D_{m}=\frac{1}{f_{1}}\left[\frac{(m+1)^{2}}{m} f_{m+1}+\left(\theta_{\mathrm{e}}-m-\frac{1}{12}\left(f_{1}-2\right)\right) f_{m}\right],
\end{aligned}
$$

where we define $f_{m}$ to be

$$
f_{m}\left(z_{\mathrm{e}}\right)=\tilde{\phi}\left(z_{\mathrm{e}}\right) \mathcal{L}_{m} \phi\left(z_{\mathrm{e}}\right)-\phi\left(z_{\mathrm{e}}\right) \mathcal{L}_{m} \tilde{\phi}\left(z_{\mathrm{e}}\right)
$$


Owing to the relations (4.16) we conclude, that the $f_{m}$ as well as $B_{m}$ and $D_{m}$ are polynomials in $f_{1}$. Since $f_{1}$ is a rational function of $z_{\mathrm{e}}$, it transforms well under modular transformations. Therefore, modular invariance is broken only by the $E_{2}$ term in $c_{m}$. We express this via the partial derivative of $c_{m}$

$$
\frac{\partial c_{m}\left(z_{\mathrm{e}}\right)}{\partial E_{2}}=-\frac{1}{12} \frac{f_{m}\left(z_{\mathrm{e}}\right)}{\phi^{2}\left(z_{\mathrm{e}}\right)} .
$$

In order to prove the holomorphic anomaly Equation (3.9), one first shows using the general results about the period integrals in [31] that the instanton part of the prepotential can be expressed by the functions $f_{m}\left(z_{\mathrm{e}}\right)$. A tedious calculation reveals

$$
\frac{1}{2 \pi \mathrm{i}} \frac{\partial}{\partial t} F^{(0)}(\tau, t)=\sum_{m \geq 1} \frac{f_{m}\left(z_{\mathrm{e}}\right)}{\phi^{2}\left(z_{\mathrm{e}}\right)} z_{1}^{m} .
$$

Using the inverse function theorem and Equations (4.22), (4.13) yields

$$
\frac{\partial z_{1}}{\partial E_{2}}=\frac{1}{12}\left(\frac{1}{2 \pi \mathrm{i}} \frac{\partial z_{1}}{\partial t}\right)\left(\frac{1}{2 \pi \mathrm{i}} \frac{\partial F^{(0)}}{\partial t}\right) .
$$

Now, we have

$$
\frac{\partial}{\partial E_{2}}\left(\frac{1}{2 \pi \mathrm{i}} \frac{\partial F^{(0)}}{\partial t}\right)=\frac{1}{12}\left(\frac{\partial^{2} F^{(0)}}{\partial(2 \pi \mathrm{i} t)^{2}}\right)\left(\frac{1}{2 \pi \mathrm{i}} \frac{\partial F^{(0)}}{\partial t}\right),
$$

which implies that up to a constant term in $p$ one arrives at

$$
\frac{\partial F^{(0)}}{\partial E_{2}}=\frac{1}{24}\left(\frac{1}{2 \pi \mathrm{i}} \frac{\partial F^{(0)}}{\partial t}\right)^{2}
$$

By definition of $F_{n}^{(0)}$, Equation (3.4), we have $\frac{1}{2 \pi \mathrm{i}} \frac{\partial}{\partial t} F^{(0)}(\tau, t)=\sum_{m \geq 1} m$ $F_{m}^{(0)} p^{m}$ and hence obtain by resummation

$$
\frac{\partial F_{n}^{(0)}}{\partial E_{2}}=\frac{1}{24} \sum_{s=1}^{n-1} s(n-s) F_{s}^{(0)} F_{n-s}^{(0)} .
$$

This almost completes the derivation of (3.9). We still need to determine the explicit form of $F_{n}^{(0)}$. To achieve this we proceed inductively. Using (4.8), 
(4.23) and (4.13) one obtains

$$
F_{1}^{(0)}=\frac{\zeta f_{1}}{\phi^{2}}=q^{\frac{1}{2}} \frac{E_{4}}{\eta^{12}} .
$$

Employing the structure (4.17) one can evaluate (4.23) and calculate that

$$
\begin{aligned}
F_{n}^{(0)} & =\frac{\zeta^{n} f_{1}^{n}}{\phi^{6 n}} P_{6 n-2}\left(E_{2}, E_{4}, E_{6}\right), \\
& =\left(\frac{\zeta f_{1}}{\phi^{2}}\right)^{n} \frac{1}{\phi^{4 n}} P_{6 n-2}\left(E_{2}, E_{4}, E_{6}\right), \\
& =\frac{q^{\frac{n}{2}}}{\eta^{12 n}} P_{6 n-2}\left(E_{2}, E_{4}, E_{6}\right),
\end{aligned}
$$

where $P_{6 n-2}$ is of weight $6 n-2$ and is decomposed out of (parts of) $Q_{m}$ 's. This establishes a derivation of the holomorphic anomaly Equation (3.9) at genus zero for the elliptic fibration over Hirzebruch surface $\mathbb{F}_{1}$ with large fibre class. We collect some results for the other fibre types in Appendix B.

\subsection{Derivation from $\mathrm{BCOV}$}

The last section provided a derivation of the anomaly Equation (3.9) for genus 0 from the mirror geometry. More fundamental is a derivation purely within the context of moduli spaces of maps from Riemann surfaces to a Calabi-Yau manifold. This is the approach taken by Bershadsky et al. [9] (BCOV) to derive holomorphic anomaly equations for genus $g n$-point correlation function with $2 g-2+n>0$. The correlation functions are given by covariant derivatives to the free energies $F^{(g)}: C_{a_{1} a_{2} \ldots a_{n}}^{(g)}=D_{a_{1}} \ldots D_{a_{n}} F^{g}$, with $D_{a}$ covariant derivatives of for sections of the bundle $\mathcal{L}^{2-2 g} \otimes \operatorname{Sym}^{n} T$, with $T$ the tangent bundle of the coupling constant moduli space, and $\mathcal{L}$ a line bundle over this space whose Chern class corresponds to $G_{a \bar{b}}$.

The holomorphic anomaly equation reads for the $n$-point functions

$$
\begin{aligned}
\bar{\partial}_{a} C_{a_{1} \ldots a_{n}}^{(g)}= & \frac{1}{2} \bar{C}_{\bar{a} \bar{b} \bar{c}} e^{2 K} G^{b \bar{b}} G^{c \bar{c}} C_{b c a_{1} \ldots a_{n}}^{(g-1)}+ \\
& +\frac{1}{2} \bar{C}_{\bar{a} \bar{b} \bar{c}} e^{2 K} G^{b \bar{b}} G^{c \bar{c}} \sum_{r=0}^{g} \sum_{s=0}^{n} \frac{1}{s !(n-s) !}
\end{aligned}
$$




$$
\begin{aligned}
& \times \sum_{\sigma \in S_{n}} F_{b a_{\sigma(1)} \ldots a_{\sigma(s)}}^{(r)} C_{c a_{\sigma(s+1)} \ldots a_{\sigma(n)}}^{(g-r)} \\
& -(2 g-2+n-1) \sum_{s=1}^{n} G_{a \bar{a}_{s}} C_{a_{1} \ldots a_{s-1} a_{s+1} \ldots a_{n}}^{(g)} .
\end{aligned}
$$

This equation can be summarized in terms of the generating function

$$
\begin{aligned}
F\left(g_{s}, t^{a} ; x^{a}\right)= & \sum_{g=0}^{\infty} \sum_{n=0}^{\infty} g_{s}^{2 g-2} \frac{1}{n !} C_{a_{1} \ldots a_{n}}^{(g)} x^{a_{1}} \ldots x^{a_{n}} \\
& +\left(\frac{\chi(M)}{24}-1\right) \log g_{s} .
\end{aligned}
$$

Contrary to [9], we take the terms with $2 g-2+n \leq 0$ as given by $D_{1} \ldots$ $D_{n} F^{(g)}$ instead of setting them to 0. Equation (4.30) implies that $F$ satisfies

$$
\begin{aligned}
\bar{\partial}_{a} \exp (F)= & {\left[\frac{g_{s}^{2}}{2} \bar{F}_{\bar{a} \bar{b} \bar{c}} e^{2 K} G^{b \bar{b}} G^{c \bar{c}} \frac{\partial^{2}}{\partial x^{b} \partial x^{c}}\right.} \\
& \left.-G_{\bar{a} b} x^{b}\left(g_{s} \frac{\partial}{\partial g_{s}}+x^{c} \frac{\partial}{\partial x^{c}}\right)\right] \exp (F) .
\end{aligned}
$$

To relate (4.32) to the holomorphic anomaly Equation (3.9) for this geometry, we split again the Kähler parameters $t^{a}$ into the fibre parameter $\tau$ and base parameters $t^{i}$. Then, we write $F\left(g_{s}, \tau, t^{i} ; x^{i}\right)$ as a Fourier expansion instead of a Taylor expansion in $x^{i}$ :

$$
\begin{aligned}
F\left(g_{s}, \tau, t^{i} ; x^{i}\right)= & \sum_{g=0}^{\infty} \sum_{\beta \in H_{2}(B, \mathbb{Z})} g_{s}^{2 g-2} F_{\beta}^{(g)}(\tau) f_{\beta}^{(g)}\left(x^{i}, t^{i}\right) \mathrm{e}^{2 \pi \mathrm{i} \beta \cdot x} p^{\beta} \\
& +\left(\frac{\chi(M)}{24}-1\right) \log g_{s},
\end{aligned}
$$

with as before $p^{\beta}=\exp \left(2 \pi \mathrm{i} t^{i} \beta_{i}\right), q=\exp (2 \pi \mathrm{i} \tau)$. Moreover, the functions $f_{\beta}^{(g)}\left(x^{i}, t^{i}\right)$ satisfy $\left.D_{i} F\right|_{x=0}=\left.\partial_{x_{i}} F\right|_{x=0}$ and $f_{\beta}^{(g)}\left(0, t^{i}\right)=1$. In the large volume limit, the covariant derivatives $D_{i}$ become flat derivatives $\frac{\partial}{\partial t^{i}}$ and thus $f_{\beta}^{g}\left(x^{i}, t^{i}\right) \rightarrow 1$. Therefore, to deduce (3.9) from (4.32) we can set $x^{i}=0$ and replace the $\frac{\partial}{\partial x^{i}}$ by $\frac{\partial}{\partial t^{i}}$. 
Equation (3.9) follows now by considering $\frac{1}{2 \pi \mathrm{i}} \partial_{\bar{\tau}} \exp (F)$ on the righthand side of (4.32). As discussed earlier, all $\bar{\tau}$ dependence arises from completing the weight 2 Eisenstein series $\widehat{E}_{2}(\tau)=E_{2}(\tau)-\frac{3}{\pi \tau_{2}}$, which gives:

$$
\frac{\partial}{\partial E_{2}}=\frac{4 \pi^{2} \tau_{2}^{2}}{3} \frac{\partial}{2 \pi \mathrm{i} \partial \bar{\tau}}
$$

We first discuss how the right-hand side of (3.9) can be derived from Equation (4.32) for the geometry $X_{18}[1,1,1,6,9]$. We use the basis $(2.7)$ and choose as parameters the "base" parameter $t=B+\mathrm{i} \mathcal{J}$ and the fibre parameter $\tau=\tau_{1}+\mathrm{i} \tau_{2}$. We are interested in the large volume limit $\tau \rightarrow \mathrm{i} \infty$, $t \rightarrow \mathrm{i} \infty$ in such a way that $\mathcal{J} \gg \tau_{2}$. In this limit, the Kähler potential is well approximated by the polynomial form

$$
K \approx-\log \left(\frac{4}{3} \tilde{d}_{a b c} \mathcal{J}^{a} \mathcal{J}^{b} \mathcal{J}^{c}\right)=-\log \left(\frac{4}{3}\left(\alpha \tau_{2}^{3}+3 \tau_{2} \mathcal{J}^{2}\right)\right),
$$

with $\alpha=\tilde{\mathcal{K}}_{\mathrm{e}}^{3}(2.7)$. This gives for the metric

$$
\left(\begin{array}{cc}
G_{\tau \bar{\tau}} & G_{t \bar{\tau}} \\
G_{\tau \bar{t}} & G_{t \bar{t}}
\end{array}\right) \approx\left(\begin{array}{cc}
\frac{1}{4 \tau_{2}^{2}} & \frac{\alpha \tau_{2}}{3 \mathcal{J}^{3}} \\
\frac{\alpha \tau_{2}}{3 \mathcal{J}^{3}} & \frac{1}{2 \mathcal{J}^{2}}
\end{array}\right),
$$

which gives for the matrix $e^{K} G^{i \bar{j}}$

$$
e^{K} G^{-1} \approx\left(\begin{array}{cc}
\frac{1}{\mathcal{J}^{2}} & -\frac{2 \alpha \tau_{2}^{2}}{3 \mathcal{J}^{3}} \\
-\frac{\alpha \tau_{2}^{2}}{3 \mathcal{J}^{3}} & \frac{1}{2 \tau_{2}}
\end{array}\right) .
$$

Thus in the limit $\mathcal{J} \rightarrow \infty$, one finds that only $e^{K} G^{t \bar{t}} \approx \frac{1}{2 \tau_{2}}$ does not vanish. Therefore, $\bar{C}_{\bar{\tau} \bar{b} \bar{c}} e^{2 K} G^{b \bar{b}} G^{c \bar{c}} \frac{\partial^{2}}{\partial x^{b} \partial x^{c}} \approx \frac{1}{4 \pi^{2}} \frac{1}{4 \tau_{2}^{2}} \frac{\partial^{2}}{\partial x^{t} \partial x^{t}} .{ }^{4}$ Using (4.34), this shows that (4.32) reduces to:

$$
\frac{\partial}{\partial E_{2}} \exp (F)=\frac{g_{s}^{2}}{24}\left(p \frac{\partial}{\partial p}\right)^{2} \exp (F)
$$

Expansion of both sides in $p$ and taking the $p^{n}$ coefficient gives a holomorphic anomaly Equation as (3.9) for $g=0$. It also gives the correct (3.9) for $g>0$ except for the appearance of $K_{B}$. We belief that a more thorough

\footnotetext{
${ }^{4}$ The factor $\frac{1}{4 \pi^{2}}$ appears due to a factor $-2 \pi$ i between the moduli in [9] and ours.
} 
analysis of the covariant derivatives will explain this term. Assuming the form $f_{\beta}^{(g)}(x, t) \rightarrow 1+x^{2} \beta \cdot K_{B}+\cdots$ would give the shift in (3.9).

The derivation is very similar for the other types of fibres discussed in Section 2. The right hand side of Equation (4.37) is simply divided by $a$, in agreement with [32].

\section{T-duality on the fibre}

In this section, we discuss properties of $T$-duality on the elliptic fibre in order to relate the result from our period calculations to $D 4$-brane counting. One can perform two $T$-dualities around the circles of the elliptic fibre. Due to the freedom in choosing the circles, this leads to an $S L(2, \mathbb{Z})$ (or a congruence subgroup) group of dualities mapping IIA branes to IIA branes. This duality group is equal to the modular subgroup of the monodromy group which leave invariant the $F_{\beta}^{(g)}$ 's discussed in Section 3.3.

Let $D 2_{e / \beta}$ be a $D 2$-brane wrapped either on the elliptic fibre $e$ or on a class $\beta$ in the base. Moreover, we denote by $D 4_{e}$ a D4-brane wrapped around the base and by $D 4_{\beta}$ a D4-brane wrapped around the cycle $\beta$ in the base and the elliptic fibre $e$. The double T-duality on both circles of the elliptic fibre transforms pairs of $D$-brane charges heuristically in the following way:

$$
\begin{aligned}
& \left(\begin{array}{l}
D 6 \\
D 4_{\mathrm{e}}
\end{array}\right)=\gamma\left(\begin{array}{l}
\tilde{D} 6 \\
\tilde{D} 4_{\mathrm{e}}
\end{array}\right), \\
& \left(\begin{array}{l}
D 4_{\beta} \\
D 2_{\beta}
\end{array}\right)=\gamma\left(\begin{array}{l}
\tilde{D} 4_{\beta} \\
\tilde{D} 2_{\beta}
\end{array}\right), \\
& \left(\begin{array}{l}
D 2_{e} \\
D 0
\end{array}\right)=\gamma\left(\begin{array}{l}
\tilde{D} 2_{e} \\
\tilde{D} 0
\end{array}\right),
\end{aligned}
$$

with $\gamma$ in $S L_{2}(\mathbb{Z})$ or a congruence subgroup. See for more a more formal treatment of $T$-duality on Calabi-Yau manifolds [3,4]. In the following sections, we will always consider the element $\gamma=\left(\begin{array}{cc}0 & -1 \\ 1 & 0\end{array}\right)$. T-duality is not valid for every choice of the Kähler parameter. One way to see this is that the BPS invariants of $D 2$ branes do not depend on the choice of the Kähler moduli but those of $D 4$ and $D 6$ branes do through wall-crossing. The choice where the two are related by $T$-duality is sufficiently close to the class of the elliptic fibre, this is called a suitable polarization in the literature [34]. Sufficiently close means that no wall is crossed between the fibre class and the suitable polarization.

The equality of invariants of $D 0$ branes and $D 2$ branes wrapping the fibre can be easily verified. The BPS invariant of an arbitrary number $n>0$ 
of $D 0$ branes is known to be equal to the Euler number of the CalabiYau [42, Section 6.5]:

$$
\Omega((0,0,0, n), M)=-\chi(M) .
$$

One can verify that these equal the BPS invariants of $n D 2$ branes wrapping the $E_{8}$ elliptic fibre of $M$. See for example the tables with $\mathrm{GW}$-invariants for $X_{18}(9,6,1,1,1)$ and $X_{24}(12,8,2,1,1)$ in Appendix $\mathrm{B}$ of [30]. If the modular group is a congruence subgroup of level $k$ then only the BPS invariant corresponding to $n=0 \bmod k D 2$ branes wrapping the fibre equals (5.2).

Our interest is in the $D 4$-branes which can be obtained from $D 2_{\gamma}$ with $\gamma=\beta+n e$ by $T$-duality. These $D 4$-branes wrap classes in the base times the fibre, and have $D 0$ brane charge $n$. D4-branes on Calabi-Yau manifolds correspond to black holes in four-dimensional space-time and are well studied [47]; in particular, $M$-theory relates the degrees of freedom of $D 4$-brane black holes to those of a $\mathcal{N}=(4,0)$ conformal field theory (CFT) with left and right central charges:

$$
c_{L}=P^{3}+\frac{1}{2} c_{2} \cdot P, \quad c_{R}=P^{3}+c_{2} \cdot P,
$$

with $P$ the 4-cycle wrapped by the $D 4$-brane, and $c_{2}$ the second Chern class of the Calabi-Yau threefold. Typically, the number of 2-cycles in the $D 4$-brane is larger than the number of 2-cycles in the Calabi-Yau threefold.

In the following, we will use the notation of [49]. The homology class $P$ naturally gives rise to a quadratic form $D_{a b}=d_{a b c} P^{c}$ which has signature $\left(1, b_{2}(M)-1\right)$. Let $\Lambda$ be the lattice $\mathbb{Z}^{b_{2}}$ with quadratic form $D_{a b}$. The dual lattice with quadratic form $D_{a b}^{-1}$ is denoted by $\Lambda^{*}$. The Kähler modulus $\mathcal{J}=\int_{X} \omega$ gives the natural projection of a vector $\mathbf{k} \in \Lambda$ to the positivedefinite subspace of $\Lambda \otimes \mathbb{R}$

$$
\mathbf{k}_{+}=\frac{\mathbf{k} \cdot \mathcal{J}}{\mathcal{J}^{2}} \mathcal{J}
$$

with $\mathcal{J}^{2}=d_{a b c} P^{a} \mathcal{J}^{b} \mathcal{J}^{c}$. 
The supergravity partition function of $D 4$-branes generically takes the following form $[10,19,49]$ :

$$
\begin{aligned}
\mathcal{Z}_{P}\left(C_{\mathrm{R}}, \tau ; t\right)= & \sum_{Q_{0}, Q} \bar{\Omega}(\Gamma ; t)(-1)^{P \cdot Q} \\
& \times e\left(-\bar{\tau} \hat{Q}_{\overline{0}}+\tau(Q-B)_{+}^{2} / 2+\bar{\tau}(Q-B)_{-}^{2} / 2\right. \\
& \left.+C_{\mathrm{R}} \cdot(Q-B / 2)\right) .
\end{aligned}
$$

where $P, Q$ and $Q_{0}$ correspond to the homology classes of respectively the $D 4-, D 2$ - and $D 0$-branes. The parameter $C_{\mathrm{R}} \in \Lambda \otimes \mathbb{R}$ is related to the Ramond-Ramond 3-form that couples to D2-branes: $\mathcal{C}_{\mathrm{R}, 3}=C_{\mathrm{R}}^{i} J_{i} \wedge d t$ in terms of the basis $J_{i}$ of $(1,1)$-forms on $M$ introduced below Equation (2.8). Furthermore, $\tau$ is the modular parameter valued in the upper-half plane $\mathcal{H}$, and $t=B+\mathrm{i} \mathcal{J} \in \Lambda \otimes \mathbb{C}$ the complexified Kähler modulus. On the right hand side, the coefficients $\bar{\Omega}(\Gamma ; t)$ are rational invariants and related to the integer invariants $\Omega(\Gamma ; t)$ by the multi-cover formula

$$
\bar{\Omega}(\Gamma ; t)=\sum_{m \mid \Gamma, m \geq 1} \frac{\Omega(\Gamma / m ; t)}{m^{2}} .
$$

Note that the multi-cover contributions come here with a factor $m^{-2}$, whereas the generating function $F^{(0)}\left(t^{a}\right)$ (3.1) of genus 0 Gromov-Witten invariants $\bar{n}_{\gamma}^{(0)}$ weights the Gopakumar-Vafa invariants $n_{\gamma / m}^{(0)}$, by $m^{-3}: \bar{n}_{\gamma}^{(0)}=$ $\sum_{m \mid \gamma, m \geq 1} n_{\gamma / m}^{(0)} / m^{3}$. The invariants $\Omega(\Gamma ; t)$ are related to the Euler number of the appropriate moduli space $\mathcal{M}_{t}(\Gamma)$ by

$$
\Omega(\Gamma ; t)=(-1)^{\operatorname{dim}_{\mathbb{C}} \mathcal{M}_{t}(\Gamma)} \chi\left(\mathcal{M}_{t}(\Gamma)\right) .
$$

Furthermore, the quantity $\hat{Q}_{\overline{0}}$ is a shorthand notation for $-Q_{0}+\frac{1}{2} Q^{2}$. This quantity determines the horizon area of the black hole $A_{\mathrm{bh}}=4 \pi \sqrt{\frac{2}{3} c_{R} \hat{Q}_{\overline{0}}}$, which is the classical manifestation of the entropy of the black hole.

Physical arguments imply that $\mathcal{Z}_{P}\left(C_{\mathrm{R}}, \tau ; t\right)$ transforms as a modular form of weight $\left(\frac{1}{2},-\frac{3}{2}\right)$. If the $B$-field decouples from the stability condition, $\mathcal{Z}_{P}\left(C_{\mathrm{R}}, \tau ; t\right)$ allows a theta function decomposition:

$$
\mathcal{Z}_{P}\left(C_{\mathrm{R}}, \tau ; t\right)=\sum_{\mu} \overline{h_{P, \mu}(\tau)} \Theta_{P, \mu}\left(\tau, C_{\mathrm{R}}, B\right)
$$


and $h_{P, \mu}(\tau)$ is a vector valued modular form of weight $-1-b_{2}(M) / 2$ given by

$$
h_{P, \mu}(\tau)=\sum_{Q_{0}} \Omega_{P}\left(\hat{Q}_{\overline{0}}\right) q^{\hat{Q}_{\overline{0}}}
$$

with $\hat{Q}_{\overline{0}}=-Q_{0}+\frac{1}{2} \mu^{2}$. This symmetry is also present in the MaldacenaStrominger-Witten (MSW) conformal field theory which arises in the near horizon geometry of a single centre $D 4$-brane black hole [10]. We refer to [49] for a discussion of the relation between the supergravity partition and the CFT partition function. In terms of the central charges $c_{\mathrm{L} / \mathrm{R}}$ of this conformal field theory, $h_{P, \mu}(\tau)$ typically takes the form

$$
h_{P, \mu}(\tau)=\frac{f_{P, \mu}(\tau)}{\eta(\tau)^{c_{\mathrm{R}}}}
$$

with $f_{P, \mu}(\tau)$ a vector-valued modular form of weight $-1-b_{2}(M) / 2+c_{\mathrm{R}} / 2$.

We continue by specializing to the elliptic Calabi-Yau fibrations. We denote the four-cycle obtained by T-duality from a curve $\beta_{i}$ in the base by $\beta^{i}$ or simply $\beta$. Whether $\beta$ denotes a two- or four-cycle should be clear from the context. One derives from the triple intersection numbers $(2.13)$ that these four-cycles $\beta^{i}$, have a vanishing triple intersection number $(\beta)^{3}=0$. These $D 4$-branes correspond therefore not to large black holes, but to "small" black holes. This means that only after addition of higher derivative corrections to the supergravity effective action one finds a non-vanishing area of the horizon. It is very intriguing that we can obtain detailed knowledge about the spectrum of these black holes using mirror symmetry.

Let $\iota: \beta \rightarrow M$ be the embedding of the divisor $\beta$ into $M$, which provides a pull back map on the second cohomology $\iota^{*}: H^{2}(M, \mathbb{Z}) \rightarrow H^{2}(\beta, \mathbb{Z})$. Since the divisors $\beta$ are not positive, this map is not injective. One deduces from (2.13) that the rank of the quadratic form $D_{a b}=d_{a b j} \beta^{j}$ is 2 independent of $M$. Therefore, the image of the map $\iota^{*}$ is two-dimensional, and we find consequently that the modular weight of $h_{\beta, \mu}(\tau)$ is equal to -2 . More details of the modular properties of $h_{\beta, \mu}(\tau)$ can be derived. If $\beta$ is primitive, i.e., $\beta / n \notin H_{4}(M, \mathbb{Z})$ for $n \geq 2$, one can find another divisor $\tilde{\beta}$ such that $\mathcal{K} \cdot \beta$. $\tilde{\beta}=1$. The quadratic form $D_{a b}$ then takes the form:

$$
\left(\begin{array}{cc}
0 & 1 \\
1 & \mathcal{K} \cdot \tilde{\beta}^{2}
\end{array}\right)
$$


With this information one can precisely determine the modular transformation properties of the vector-valued modular form $h_{\beta, \mu}(\tau)$. See Equation (4.17) of [48]. The elements of the modular vector are modular forms of a congruence subgroup $\Gamma(m)$.

The genus 0 free energies $F_{\beta}^{(0)}(3.8)$ give a prediction $h_{\beta, \mathbf{0}}^{\mathrm{pr}}(\tau)$ for $h_{\beta, \mathbf{0}}(\tau)$. Correcting the power by which non-primitive charges are weighted, we find for $h_{\beta, \mathbf{0}}^{\mathrm{pr}}(\tau)$ in terms of $F_{\beta}^{(0)}(\tau)$ :

$$
h_{\beta, \mathbf{0}}^{\mathrm{pr}}(\tau)=\sum_{m \mid \beta} \frac{1}{m^{2}}\left(\sum_{n \mid m} \frac{\mu(n)}{n}\right) F_{\beta / m}^{(0)}(m \tau),
$$

where $\mu(m)$ is the Möbius function, which is defined for $m \geq 1$ by $\mu(m)=$ $(-1)^{\ell}$ if $m$ is the product of $\ell$ distinct primes $\geq 2$ and $\mu(m)=0$ otherwise). The modular properties of $h_{P, \mathbf{0}}(\tau)$ defined this way are precisely consistent with the structure found for the genus 0 amplitudes obtained from the mirror periods, see Equation (3.8) combined with Equation (2.8). As explained in Section 3 , the free energy $F_{\beta}^{(0)}$ is a modular form of weight -2 , in agreement with the weight of $h_{\beta, \mu}(\tau)$. Due to contributions to $h_{\beta, 0}^{\mathrm{pr}}(\tau)(5.12)$ of $F_{\beta}^{(0)}(m \tau)$ with $m>1, h_{\beta, 0}^{\mathrm{pr}}(\tau)$ is in general an element of the congruence subgroup $\Gamma(m)$ in agreement with the analysis of the modular properties of (5.5). Generically, one cannot determine uniquely from $h_{\beta, 0}^{\mathrm{pr}}(\tau)$ the other elements of the modular vector, but in simple examples this can be done.

Besides verifying that the modular properties of $h_{\beta, \mathbf{0}}^{\mathrm{pr}}(\tau)$ agree with $h_{\beta, 0}(\tau)$, it is also possible to verify the agreement for the first few coefficients, for small $D 0$ and $D 4$-brane charge, the BPS invariants can be computed either from the microscopic $D$-brane perspective or the supergravity context $[5,11,16,19,22,23,25,52,54]$. For example from the microscopic point of view, the moduli space of a single $D 4$-brane is given by projective space $\mathbb{P}^{n}$. Using index theorems one can compute that $n=\frac{1}{6} P^{3}+\frac{1}{12} c_{2} \cdot P-1[47]$. Therefore, the first coefficient of $h_{P, \mathbf{0}}(\tau)$ is expected to be

$$
\Omega_{P}\left(-\frac{1}{24} c_{R}\right)=\frac{1}{6} P^{3}+\frac{1}{12} c_{2} \cdot P .
$$

The second coefficient corresponds to adding a unit of (anti) D0-brane charge. Now the linear system for the divisor of the $D 4$-brane is constrained to pass through the D0-brane. This gives with Equation (5.2) [22]

$$
\Omega_{P}\left(1-\frac{1}{24} c_{R}\right) \cong \chi(M)\left(\frac{1}{12} c_{2} \cdot P-1\right) .
$$




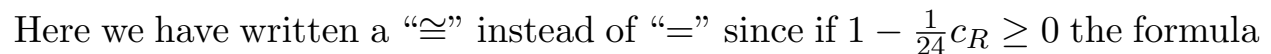
for the horizon area (given below Equation (5.7)) gives a positive value, such that the BPS states might correspond to black holes with intrinsically gravitational degrees of freedom, which are less well understood.

Continuing with two units of $\overline{D 0}$ charge, one finds

$$
\Omega_{P}\left(2-\frac{1}{24} c_{R}\right) \cong \frac{1}{2} \chi(M)(\chi(M)+5)\left(\frac{1}{12} c_{2} \cdot P-2\right) .
$$

One can in principle continue along these lines, which becomes increasingly elaborate for three reasons. First effects of $D 2$-branes become important, second single centre black holes contribute for $\hat{Q}_{\overline{0}}>0$ and third the index might depend on the background moduli $t$.

We now briefly explain which bound states appear in the supergravity picture for small $D 0 / 4$-brane charge. The first terms in the $q$-expansion cannot correspond to single centre black holes since $\hat{Q}_{\overline{0}}<0$. The first terms correspond to bound states of $D 6$ and $\overline{D 6}$-branes [19]. If $P$ is an irreducible cycle (i.e., it cannot be written as $P=P_{1}+P_{2}$ with $P_{1}$ and $P_{2}$ effective classes) then the charges $\Gamma_{1}$ and $\Gamma_{2}$ of the constituents are

$$
\Gamma_{1}=\left(1, P, \frac{1}{2} P^{2}-\frac{c_{2}}{24}, \frac{1}{6} P^{3}+\frac{c_{2} \cdot P}{24}\right), \quad \Gamma_{2}=\left(-1,0, \frac{c_{2}}{24}, 0\right),
$$

The index of a 2-centre bound state is given by:

$$
\left\langle\Gamma_{1}, \Gamma_{2}\right\rangle \Omega\left(\Gamma_{1}\right) \Omega\left(\Gamma_{2}\right),
$$

with $\left\langle\Gamma_{1}, \Gamma_{2}\right\rangle=-P_{1}^{0} Q_{0,2}+P_{1} \cdot Q_{2}-P_{2} \cdot Q^{1}+P_{2}^{0} Q_{0,1}$ the symplectic inner product. Since the constituents are single D6-branes with a non-zero flux, their index is $\Omega\left(\Gamma_{i} ; t\right)=1$. Therefore, $\Omega_{P}\left(-\frac{1}{24} c_{R}\right)=\left\langle\Gamma_{1}, \Gamma_{2}\right\rangle=\frac{1}{6} P^{3}+\frac{1}{12} c_{2}$. $P$, which reproduces Equation (5.13).

One can continue in a similar fashion with adding other constituents to compute indices with higher charge. For example, BPS states with charge $\Gamma=\left(0,2 P, 0, \frac{1}{3} P^{3}+\frac{c_{2} \cdot P}{12}\right)$ corresponds to $\Gamma_{1}$ as in $(5.16)$ and

$$
\Gamma_{2}=\left(-1, P,-\frac{1}{2} P^{2}+\frac{c_{2}}{24}, \frac{1}{6} P^{3}+\frac{c_{2} \cdot P}{24}\right) .
$$

One obtains then $\Omega_{2 P}\left(-\frac{1}{24} c_{R}\right)=\frac{8}{6} P^{3}+\frac{2}{12} c_{2} \cdot P$. Similarly, one could also add $\overline{D 0}$ charges, and find the right hand sides of Equations (5.13) to (5.15) with $P$ replaced by $2 P$. 
Example: $X_{18}[1,1,1,6,9]$

We now consider the periods for $X_{18}[1,1,1,6,9]$, i.e., a elliptic fibration over $\mathbb{P}^{2}$ and compare with the above discussion. This Calabi-Yau has a two-dimensional Kähler cone, and lends it self well to studies of D4-branes. We consider D4-branes wrapping the divisor whose Poincare dual is the hyperplane class $H$ of the base surface $\mathbb{P}^{2}$. The number of wrappings is denoted by $r$.

As explained in Section 3, the genus 0 Gromov-Witten invariants are well-studied [14,30]. Using $(5.12)$, the $F_{\beta}^{(0)}(\tau)$ provide the following predictions for $h_{n H, \mathbf{0}}(\tau)$ :

$$
\begin{aligned}
h_{H, \mathbf{0}}^{\mathrm{pr}}(\tau)= & \frac{31 E_{4}^{4}+113 E_{4} E_{6}^{2}}{48 \eta(\tau)^{36}} \\
= & q^{-3 / 2}\left(3-1080 q+143770 q^{2}+204071184 q^{3}+\cdots\right), \\
h_{2 H, \mathbf{0}}^{\mathrm{pr}}(\tau)= & \frac{-196319 E_{4} E_{6}^{5}-755906 E_{4}^{4} E_{6}^{3}-208991 E_{4}^{7} E_{6}}{221184 \eta(\tau)^{72}} \\
& -\frac{1}{24} E_{2} h_{H, 0}^{\mathrm{pr}}(\tau)^{2}+\frac{1}{8} h_{H, 0}^{\mathrm{pr}}(2 \tau) \\
= & q^{-3}\left(-6+2700 q-574560 q^{2}+\cdots\right)+\frac{1}{4} h_{H, 0}^{\mathrm{pr}}(2 \tau), \\
h_{3 H, \mathbf{0}}^{\mathrm{pr}}(\tau)= & q^{-9 / 2}\left(27-17280 q+5051970 q^{2}+\cdots\right)+\frac{1}{9} h_{H, 0}^{\mathrm{pr}}(3 \tau) .
\end{aligned}
$$

We want to compare this to the expressions derived above from the point of view of $D 4$-branes. For $r=1$, we have

$$
\Omega(\Gamma ; \mathcal{J})=\frac{1}{12} c_{2} \cdot H=3,
$$

in agreement with the first coefficient of $h_{H}(\tau)$. The second term in the $q$-expansion corresponds to

$$
\Omega\left(1, \frac{1}{2} H,-1\right)=\chi(M)\left(\frac{1}{12} c_{2} \cdot P-1\right)=1080,
$$

which is also in agreement with the periods. For two $\bar{D} 0$ branes, we find a small discrepancy, one finds:

$$
\frac{1}{2}\left(\frac{1}{12} c_{2} \cdot P-2\right) \chi(M)(\chi(M)+5)=144450 .
$$

This is an excess of $1080=-2 \chi(M)$ states compared to the $3 \mathrm{rd}$ coefficient in $h_{1}(\tau)$. This number is very suggestive of a bound state picture, possibly 
involving $D 2$ branes. Since $\hat{Q}_{\overline{0}}>0$ one could argue that these states are due to intrinsic gravitational degrees of freedom, but it seems actually a rather generic feature if we consider other elliptic fibrations (e.g., over $\mathbb{F}_{1}$ ).

For $r=2$, also the first two coefficients of the spectrum match with the $D 4$-brane indices, and the 3rd differs by $-6 \chi(M)$. Something non-trivial happens for $r=3$. We leave an interpretation of these indices from multicentre solutions for a future publication, and continue with the example of the local elliptic surface [57].

\section{BPS invariants of the rational elliptic surface}

This section continues with the comparison of the $D 4$ - and $D 2$-brane spectra for the $E_{8}$ elliptic fibration over the Hirzebruch surface $\mathbb{F}_{1}$, which was first addressed by Refs. [57,63]. Let $\sigma: \mathbb{F}_{1} \rightarrow M$ be the embedding of $\mathbb{F}_{1}$ into the Calabi-Yau threefold. The surface $\mathbb{F}_{1}$ is itself a fibration $\pi: \mathbb{F}_{1} \rightarrow C \cong \mathbb{P}^{1}$ with fibre $f \cong \mathbb{P}^{1}$, with intersections $C^{2}=-1, C \cdot f=1$ and $f^{2}=0$. The Kähler cone of $M$ is spanned by the elliptic fibre class $J_{1}$, and the classes $J_{2}=\sigma_{*}(C+f)$ and $J_{3}=\sigma_{*}(f)$. The Calabi-Yau intersections and Chern classes are given by (2.17).

A few predictions from the periods for the $D 4$-brane partition functions are

$$
\begin{aligned}
h_{C, \mathbf{0}}^{\mathrm{pr}}(\tau) & =\frac{E_{4}(\tau)}{\eta(\tau)^{12}}=q^{-1 / 2}(1+252 q+\cdots), \\
h_{f, \mathbf{0}}^{\mathrm{pr}}(\tau) & =\frac{2 E_{4}(\tau) E_{6}(\tau)}{\eta(\tau)^{24}} \\
& =-2 q^{-1}+480+282888 q+\cdots, \\
h_{2 C, \mathbf{0}}^{\mathrm{pr}}(\tau) & =\frac{E_{2}(\tau) E_{4}(\tau)^{2}+2 E_{4}(\tau) E_{6}(\tau)}{24 \eta(\tau)^{24}}+\frac{1}{8} h_{C, 0}^{\mathrm{pr}}(2 \tau) \\
& =-9252 q-673760 q^{2}+\cdots+\frac{1}{4} h_{C}(2 \tau), \\
h_{3 C, \mathbf{0}}^{\mathrm{pr}}(\tau) & =\frac{54 E_{2}^{2} E_{4}^{3}+216 E_{2} E_{4}^{2} E_{6}+109 E_{4}^{4}+197 E_{4} E_{6}^{2}}{15552 \eta^{36}}+\frac{2}{27} h_{C, 0}^{\mathrm{pr}}(3 \tau) \\
& =848628 q^{3 / 2}+115243155 q^{5 / 2}+\cdots+\frac{1}{9} h_{C, 0}^{\mathrm{pr}}(3 \tau) .
\end{aligned}
$$

Since $c_{2}(M) \cdot f=24$, explicit expressions in terms of modular forms for the divisors $h_{C+n f}(\tau)$ become rather lengthy. Interestingly, one finds that for this class the first coefficients (checked up to $n=12$ ), are given by $1+2 n$ in agreement with Equation (5.13). Moreover, the second and third coefficients 
are respectively given by $\chi(M)\left(\frac{1}{12} c_{2} \cdot P-1\right)$ and $\frac{1}{2} \chi(\chi+9)\left(\frac{1}{12} c_{2} \cdot P-2\right)$ as long as the corresponding $\hat{Q}_{\overline{0}}<0$.

Another interesting class are $r D 4$ branes wrapped on the divisor $C$, which is however not an ample divisor since $C=J_{2}-J_{3}$. The Euler number of this divisor is $c_{2} \cdot C=12$, it is in fact the rational elliptic surface $\mathbb{F}_{9}$, which is the 9-point blow-up of the projective plane $\mathbb{P}^{2}$, or equivalently, the 8-point blow-up of $\mathbb{F}_{1}$.

For $r D 4$ branes we have $P=r C$. Equation (2.17) shows that the quadratic form $D_{a b c} P^{c}$ restricted to $J_{1}$ and $J_{3}$ is

$$
r\left(\begin{array}{ll}
1 & 1 \\
1 & 0
\end{array}\right)
$$

The other 8 independent classes of $H^{2}(P, \mathbb{Z})$ are not "visible" to the computation based on periods, since these two-cycles of $P$ do not pull back to 2-cycles of $M$. We continue by confirming the expressions found from the periods with a computation of the Euler numbers of the moduli spaces of semi-stable sheaves as in $[57,63]$. The algebraic computations are more naturally performed in terms of Poincaré polynomials, and thus give more refined information about the moduli space [55]. Moreover, the eight independent classes which are not visible from the Calabi-Yau point of view, can be distinguished from this perspective.

One might wonder whether the extra parameter appearing with the Poincaré polynomial is related to the higher genus expansion of topological strings. However, the refined information of the genus expansion is different. Roughly speaking, the $D 2$-brane moduli space is a torus fibration over a base manifold [28]. The genus expansion captures the cohomology of the torus, whereas the $D 4$-brane moduli space gives naturally the cohomology of the total moduli space. For $r=1,[32]$ argues that the torus fibration is also present for moduli spaces of rank 1 sheaves on $\mathbb{F}_{9}$, but it is non-trivial to continue this to higher rank. Another approach to verify the Fourier-Mukai transform at a refined level is consider the refined topological string partition function with parameters $\epsilon_{1}$ and $\epsilon_{2}$, and then take the Nekrasov-Shatashvili limit $\epsilon_{1}=0, \epsilon_{2} \ll 1$ instead of the topological string limit $\epsilon_{1}=-\epsilon_{2}=g_{s}$.

The structure described in Section 5 for D4-brane partition functions simplifies when one specializes to a (local) surface. The charge vector $\Gamma$ becomes $\left(r, \mathrm{ch}_{1}, \mathrm{ch}_{2}\right)$ with $r$ the ranks and $\operatorname{ch}_{i}$ the Chern characters of the sheaf. Other frequently occurring quantities are the determinant $\Delta=$ $\frac{1}{r}\left(c_{2}-\frac{r-1}{2 r} c_{1}^{2}\right)$, and $\mu=c_{1} / r \in H^{2}(S, \mathbb{Q})$. In terms of the Poincaré polynomial $p(\mathcal{M}, w)=\sum_{i=0}^{2 \operatorname{dim}_{\mathbb{C}}(\mathcal{M})} b_{i}(\mathcal{M}) w^{i}$ of the moduli space $\mathcal{M}$, the (refined) 
BPS invariant reads

$$
\Omega(\Gamma, w ; \mathcal{J}):=\frac{w^{-\operatorname{dim}_{\mathbb{C}} \mathcal{M}_{\mathcal{J}}(\Gamma)}}{w-w^{-1}} p\left(\mathcal{M}_{\mathcal{J}}(\Gamma), w\right) .
$$

In the case of surfaces, a formula is available for the dimension of the moduli space

$$
\operatorname{dim}_{\mathbb{C}} \mathcal{M}_{\mathcal{J}}(\Gamma)=2 r^{2} \Delta-r^{2} \chi\left(\mathcal{O}_{S}\right)+1
$$

One can verify that the Poincaré polynomials computed later in this section are in agreement with this formula.

The rational invariant corresponding to $\Omega(\Gamma, w ; \mathcal{J})$ is $[51]$

$$
\bar{\Omega}(\Gamma, w ; \mathcal{J})=\sum_{m \mid \Gamma} \frac{\Omega\left(\Gamma / m,-(-w)^{m} ; \mathcal{J}\right)}{m} .
$$

The numerical BPS invariant $\Omega(\Gamma ; \mathcal{J})$ follows from the $\Omega(\Gamma, w ; \mathcal{J})$ by

$$
\Omega(\Gamma ; \mathcal{J})=\lim _{w \rightarrow-1}\left(w-w^{-1}\right) \Omega(\Gamma, w ; \mathcal{J})
$$

and similarly for the rational invariants $\bar{\Omega}(\Gamma ; \mathcal{J})$.

The generating function (5.5) becomes for a complex surface $S$

$$
\begin{aligned}
\mathcal{Z}_{r}(\rho, z, \tau ; S, \mathcal{J})= & \sum_{c_{1}, c_{2}} \bar{\Omega}(\Gamma, w ; \mathcal{J})(-1)^{r c_{1} \cdot K_{S}} \\
& \times \bar{q}^{r \Delta(\Gamma)-\frac{r \chi(S)}{24}-\frac{1}{2 r}\left(c_{1}+r K_{S} / 2\right)_{-}^{2}} q^{\frac{1}{2 r}\left(c_{1}+r K_{S} / 2\right)^{2}} e^{2 \pi \mathrm{i} \rho \cdot\left(c_{1}+r K_{S} / 2\right)},
\end{aligned}
$$

with $\rho \in H^{2}(S, \mathbb{C}), w=\mathrm{e}^{2 \pi \mathrm{i} z}$ and $q=\mathrm{e}^{2 \pi \mathrm{i} \tau}$. Twisting by a line bundle leads to an isomorphism of moduli spaces. It is therefore sufficient to determine $\Omega(\Gamma, w ; \mathcal{J})$ only for $c_{1} \bmod r$, and it moreover implies that $\mathcal{Z}_{r}(\rho, z, \tau ; S, \mathcal{J})$ allows a theta function decomposition as in (5.8)

$$
\mathcal{Z}_{r}(\rho, z, \tau ; S, \mathcal{J})=\sum_{\mu \in \Lambda^{*} / \Lambda} \overline{h_{r, \mu}(z, \tau ; S, \mathcal{J})} \Theta_{r, \mu}(\rho, \tau ; S)
$$

with

$$
h_{r, \mu}(z, \tau ; S, \mathcal{J})=\sum_{c_{2}} \bar{\Omega}(\Gamma, w ; \mathcal{J}) q^{r \Delta(\Gamma)-\frac{r \chi(S)}{24}}
$$


and

$$
\Theta_{r, \mu}(\rho, \tau ; S)=\sum_{\mathbf{k} \in H^{2}(S, r \mathbb{Z})+r K_{S} / 2+\mu}(-1)^{r \mathbf{k} \cdot K_{S}} q^{\mathbf{k}_{+}^{2} / 2 r} \bar{q}^{-\mathbf{k}_{-}^{2} / 2 r} \mathrm{e}^{2 \pi \mathrm{i} \rho \cdot \mathbf{k}} .
$$

Note that $\Theta_{r, \mu}(\rho, \tau ; S)$ depends on $\mathcal{J}$ through $\mathbf{k}_{ \pm}$and does not depend on $z$.

The generating function of the numerical invariants $\Omega(\Gamma ; \mathcal{J})$ follows simply from Equation (6.4)

$$
\mathcal{Z}_{r}(\rho, \tau ; S, \mathcal{J})=\lim _{z \rightarrow \frac{1}{2}}\left(w-w^{-1}\right) \mathcal{Z}_{r}(z, \rho, \tau ; S, \mathcal{J})
$$

Physical arguments imply that this function transforms as a multivariable Jacobi form of weight $\left(\frac{1}{2},-\frac{3}{2}\right)[48,59]$ with a non-trivial multiplier system. For rank $>1$, this is only correct after the addition of a suitable nonholomorphic term $[57,59]$.

This section verifies the agreement of the BPS invariants obtained from the periods and vector bundles for $h_{r, c_{1}}\left(z, \tau ; \mathbb{F}_{9}, \mathcal{J}_{m, n}\right)$ for $r \leq 3$. The results for $r \leq 2$ are due to Göttsche [25] and Yoshioka [63]. The computations apply notions and techniques from algebraic geometry as Gieseker stability, Harder-Narasimhan filtrations and the blow-up formula. We refer to $[51,55]$ for further references and details. The most crucial difference between the computations for $\mathbb{F}_{9}$ and those for Hirzebruch surfaces in $[51,55]$ is that the lattice arising from $H^{2}\left(\mathbb{F}_{9}, \mathbb{Z}\right)$ is now ten-dimensional. We continue therefore with giving a detailed description of different bases of $H^{2}\left(\mathbb{F}_{9}, \mathbb{Z}\right)$, gluing vectors and theta functions.

\subsection{The lattice $H^{2}\left(\mathbb{F}_{9}, \mathbb{Z}\right)$}

The second cohomology $H^{2}\left(\mathbb{F}_{9}, \mathbb{Z}\right)$ gives naturally rise to a unimodular basis, it is in fact isomorphic to the unique unimodular lattice with signature $(1,9)$, which we denote by $\Lambda_{1,9}$. For this paper, three different bases $(\mathbf{C}, \mathbf{D}$ and $\mathbf{E})$ of $\Lambda_{1,9}$ are useful. The first basis is the geometric basis $\mathbf{C}$, which keeps manifest that $\mathbb{F}_{9}$ is the 9 -point blow-up of the projective plane $\mathbb{P}^{2}$. The basis vectors of $\mathbf{C}$ are $H$ (the hyperplane class of $\mathbb{P}^{2}$ ) and $\mathbf{c}_{i}$ (the exceptional divisors of the blow-up). ${ }^{5}$ The quadratic form is $\operatorname{diag}(1,-1, \ldots,-1)$. The

\footnotetext{
${ }^{5}$ We will use in general boldface to parameterize vectors.
} 
canonical class $K_{9}$ of $\mathbb{F}_{9}$ is given in terms of this basis by

$$
K_{9}=-3 H+\sum_{i=1}^{9} \mathbf{c}_{i} .
$$

One can easily verify that $K_{9}^{2}=0$. Note that $-K_{9}$ is numerically effective but not ample.

The second basis $\mathbf{D}$ parameterizes $\Lambda_{1,9}$ as a gluing of the two nonunimodular lattices $A$ and $D$. The basis $\mathbf{D}$ is given in terms of $\mathbf{C}$ by

$$
\begin{aligned}
& \mathbf{a}_{1}=-K_{9}, \quad \mathbf{a}_{2}=H-\mathbf{c}_{9}, \\
& \mathbf{d}_{i}=\mathbf{c}_{i}-\mathbf{c}_{i+1}, \quad 1 \leq i \leq 7, \\
& \mathbf{d}_{8}=-H+\mathbf{c}_{7}+\mathbf{c}_{8}+\mathbf{c}_{9} .
\end{aligned}
$$

The $\mathbf{a}_{i}$ are basis elements of $A$ and $\mathbf{d}_{i}$ of $D$. Since $A$ and $D$ are not unimodular, integral lattice elements of $\mathbf{C}$ do not correspond to integral elements of $D$. For example, $\mathbf{c}_{9}$ is given by

$$
\mathbf{c}_{9}=\frac{1}{2}\left(\mathbf{a}_{1}+\mathbf{a}_{2}+\sum_{i=1}^{6} i \mathbf{d}_{i}+3 \mathbf{d}_{7}+4 \mathbf{d}_{8}\right) .
$$

The other $\mathbf{c}_{i}$ are easily determined using $\mathbf{c}_{9}$. The quadratic form $\mathcal{Q}_{A}$ of the lattice $A$ is

$$
\mathcal{Q}_{A}=\left(\begin{array}{ll}
0 & 2 \\
2 & 0
\end{array}\right),
$$

and $\mathcal{Q}_{D}$ of the lattice $D$ is minus the $D_{8}$ Cartan matrix

$$
\mathcal{Q}_{D}=-\mathcal{Q}_{D_{8}}=-\left(\begin{array}{cccccccc}
2 & -1 & 0 & 0 & 0 & 0 & 0 & 0 \\
-1 & 2 & -1 & 0 & 0 & 0 & 0 & 0 \\
0 & -1 & 2 & -1 & 0 & 0 & 0 & 0 \\
0 & 0 & -1 & 2 & -1 & 0 & 0 & 0 \\
0 & 0 & 0 & -1 & 2 & -1 & 0 & 0 \\
0 & 0 & 0 & 0 & -1 & 2 & -1 & -1 \\
0 & 0 & 0 & 0 & 0 & -1 & 2 & 0 \\
0 & 0 & 0 & 0 & 0 & -1 & 0 & 2
\end{array}\right) .
$$

Gluing of $A$ and $D$ to obtain $\Lambda_{1,9}$ corresponds to an isomorphism between $A^{*} / A$ and $D^{*} / D$. This isomorphism is given by 4 gluing vectors $\boldsymbol{g}_{i}$, since 
the discriminants of $A$ and $D$ are equal to 4 . We choose them to be

$$
\begin{aligned}
& \boldsymbol{g}_{0}=\mathbf{0}, \\
& \boldsymbol{g}_{1}=\frac{1}{2}(1,0,1,0,1,0,1,0,0,1), \\
& \boldsymbol{g}_{2}=\frac{1}{2}(0,1,0,0,0,0,0,0,1,1), \\
& \boldsymbol{g}_{3}=\frac{1}{2}(1,1,1,0,1,0,1,0,1,0)
\end{aligned}
$$

Theta functions which sum over $D$ will play an essential role later in this section. The theta functions $\Theta_{r D_{8}, \mu}(\tau)$ are defined by

$$
\Theta_{r D_{8}, \mu}(\tau)=\sum_{\mathbf{k}=\boldsymbol{\mu}} q_{\bmod r \mathbb{Z}} q^{\frac{\mathbf{k}^{2}}{2 r}}
$$

Such sums converge rather slowly. Therefore, we also give their expression in terms of unary theta functions $\theta_{i}(\tau)=\theta_{i}(0, \tau)$ (defined in Appendix C). For $r=1$ and the glue vectors $\boldsymbol{g}_{i}$, one has

$$
\begin{aligned}
& \Theta_{D_{8}, \boldsymbol{g}_{0}}(\tau)=\frac{1}{2}\left(\theta_{3}(\tau)^{8}+\theta_{4}(\tau)^{8}\right), \\
& \Theta_{D_{8}, \boldsymbol{g}_{1}}(\tau)=\frac{1}{2} \theta_{2}(\tau)^{8} \\
& \Theta_{D_{8}, \boldsymbol{g}_{2}}(\tau)=\frac{1}{2}\left(\theta_{3}(\tau)^{8}-\theta_{4}(\tau)^{8}\right), \\
& \Theta_{D_{8}, \boldsymbol{g}_{3}}(\tau)=\frac{1}{2} \theta_{2}(\tau)^{8} .
\end{aligned}
$$

For $r=2$, the $\mu$ in the $\Theta_{2 D_{8}, \mu}(\tau)$ take values in $D / 2 D$. The $2^{8}$ elements are naturally grouped in six classes with multiplicities 1, 56, 140, 1, 56 and two depending on the corresponding theta function $\Theta_{2 D_{8}, \mu}(\tau)$. We choose as representative for each class

$$
\begin{aligned}
\boldsymbol{d}_{0} & =\mathbf{0}, \\
\boldsymbol{d}_{1} & =(1,0,0,0,0,0,0,0), \\
\boldsymbol{d}_{2} & =(1,0,1,0,0,0,0,0), \\
\boldsymbol{d}_{3} & =(0,0,0,0,0,0,1,1), \\
\boldsymbol{d}_{4} & =(1,0,1,0,1,0,0,0), \\
\boldsymbol{d}_{5} & =(1,0,1,0,1,0,1,0),
\end{aligned}
$$

Elements $\boldsymbol{\mu} \in \boldsymbol{g}_{i}+D / 2 D$ fall similarly in conjugacy classes corresponding to their theta functions. We let $m_{i, j}$ denote the number of elements in the class represented by $\boldsymbol{g}_{i}+\boldsymbol{d}_{j}$. The non-vanishing $m_{i, j}$ are given in table 2 . 
Table 2: The number of elements $m_{i, j}$ in $\boldsymbol{g}_{i}+D / 2 D$ with equal theta functions $\Theta_{2 D_{8}, \boldsymbol{g}_{i}+\boldsymbol{d}_{j}}(\tau)$.

\begin{tabular}{lcccccc}
\hline$m_{i, j}$ & 0 & 1 & 2 & 3 & 4 & 5 \\
\hline 0 & 1 & 56 & 140 & 1 & 56 & 2 \\
1 & 128 & & & 128 & & \\
2 & 16 & 112 & 112 & & 16 & \\
3 & 128 & & & 128 & &
\end{tabular}

The corresponding theta functions are given by

$$
\begin{aligned}
& \Theta_{2 D_{8}, \boldsymbol{d}_{0}}(\tau)=\frac{1}{2}\left(\theta_{3}(2 \tau)^{8}+\theta_{4}(2 \tau)^{8}\right) \\
& \Theta_{2 D_{8}, \boldsymbol{d}_{1}}(\tau)=\frac{1}{16}\left(\theta_{3}(\tau)^{8}-\theta_{4}(\tau)^{8}\right)-\frac{1}{2} \theta_{2}(2 \tau)^{6} \theta_{3}(2 \tau)^{2} \\
& \Theta_{2 D_{8}, \boldsymbol{d}_{2}}(\tau)=\frac{1}{32} \theta_{2}(\tau)^{8} \\
& \Theta_{2 D_{8}, \boldsymbol{d}_{3}}(\tau)=\frac{1}{2}\left(\theta_{3}(2 \tau)^{8}-\theta_{4}(2 \tau)^{8}\right) \\
& \Theta_{2 D_{8}, \boldsymbol{d}_{4}}(\tau)=\frac{1}{2} \theta_{2}(2 \tau)^{6} \theta_{3}(2 \tau)^{2} \\
& \Theta_{2 D_{8}, \boldsymbol{d}_{5}}(\tau)=\frac{1}{2} \theta_{2}(2 \tau)^{8}
\end{aligned}
$$

For $\boldsymbol{g}_{1}$

$$
\begin{aligned}
\Theta_{2 D_{8}, \boldsymbol{g}_{1}}(\tau) & =\frac{1}{8} \theta_{2}(\tau)^{4}\left(\theta_{3}(2 \tau)^{4}-\frac{1}{2} \theta_{4}(2 \tau)^{4}\right), \\
\Theta_{2 D_{8}, \boldsymbol{g}_{1}+\boldsymbol{d}_{3}}(\tau) & =\Theta_{2 D_{8}, \boldsymbol{d}_{2}}(\tau),
\end{aligned}
$$

for $\boldsymbol{g}_{2}$

$$
\begin{aligned}
\Theta_{2 D_{8}, \boldsymbol{g}_{2}}(\tau) & =\frac{1}{4} \theta_{2}(\tau)^{2} \theta_{3}(2 \tau)^{6}, \\
\Theta_{2 D_{8}, \boldsymbol{g}_{2}+\boldsymbol{d}_{1}}(\tau) & =\frac{1}{16} \theta_{2}(\tau)^{6} \theta_{3}(2 \tau)^{2} \\
\Theta_{2 D_{8}, \boldsymbol{g}_{2}+\boldsymbol{d}_{2}}(\tau) & =\frac{1}{16} \theta_{2}(\tau)^{6}\left(\theta_{3}(2 \tau)^{2}-\theta_{4}(\tau)^{2}\right), \\
\Theta_{2 D_{8}, \boldsymbol{g}_{2}+\boldsymbol{d}_{4}}(\tau) & =\frac{1}{4} \theta_{2}(2 \tau)^{6} \theta_{2}(\tau)^{2},
\end{aligned}
$$

and for $\boldsymbol{g}_{3}$ :

$$
\begin{aligned}
\Theta_{2 D_{8}, \boldsymbol{g}_{3}}(\tau) & =\Theta_{2 D_{8}, \boldsymbol{g}_{1}}(\tau), \\
\Theta_{2 D_{8}, \boldsymbol{g}_{3}+\boldsymbol{d}_{3}}(\tau) & =\Theta_{2 D_{8}, \boldsymbol{g}_{1}+\boldsymbol{d}_{1}}(\tau) .
\end{aligned}
$$

The third basis is basis $\mathbf{E}$ corresponding to the representation of $\Lambda_{1,9}$ as the direct sum of the two lattices $B$ and $E$, whose basis vectors $\mathbf{b}_{i}$ 
and $\mathbf{e}_{i}$ are

$$
\begin{aligned}
& \mathbf{b}_{1}=-K_{9}, \quad \mathbf{b}_{2}=\mathbf{c}_{9}, \\
& \mathbf{e}_{i}=\mathbf{c}_{i}-\mathbf{c}_{i+1}, \quad 1 \leq i \leq 7, \\
& \mathbf{e}_{8}=-H+\mathbf{c}_{6}+\mathbf{c}_{7}+\mathbf{c}_{8} .
\end{aligned}
$$

The element $H$ of basis $\mathbf{C}$ is in terms of this basis: $H=(3,3,3,6,9,12,15$, $10,5,2)$. The intersection numbers for $\mathbf{b}_{i}$ are $\mathbf{b}_{1}^{2}=0, \mathbf{b}_{2}^{2}=-1$ and $\mathbf{b}_{1} \cdot \mathbf{b}_{2}=$ 1. The quadratic form $\mathcal{Q}_{E}$ for $E$ is minus the $E_{8}$ Cartan matrix, which is given by

$$
\left(\begin{array}{cccccccc}
2 & -1 & 0 & 0 & 0 & 0 & 0 & 0 \\
-1 & 2 & -1 & 0 & 0 & 0 & 0 & 0 \\
0 & -1 & 2 & -1 & 0 & 0 & 0 & 0 \\
0 & 0 & -1 & 2 & -1 & 0 & 0 & 0 \\
0 & 0 & 0 & -1 & 2 & -1 & 0 & -1 \\
0 & 0 & 0 & 0 & -1 & 2 & -1 & 0 \\
0 & 0 & 0 & 0 & 0 & -1 & 2 & 0 \\
0 & 0 & 0 & 0 & -1 & 0 & 0 & 2
\end{array}\right)
$$

The 256 elements in $E / 2 E$ fall in 3 inequivalent Weil orbits with vectors of length 0,2 and 4, which have multiplicities $m_{0}=1, m_{1}=120$ and $m_{2}=135$ respectively. We choose as representatives

$$
\begin{aligned}
& \boldsymbol{e}_{0}=\mathbf{0}, \\
& \boldsymbol{e}_{1}=(1,0,0,0,0,0,0,0), \\
& \boldsymbol{e}_{2}=(1,0,1,0,0,0,0,0) .
\end{aligned}
$$

The corresponding theta functions $\Theta_{r E_{8}, \boldsymbol{e}_{0}}$ are for $r=1,2$ :

$$
\begin{aligned}
& \Theta_{E_{8}, \boldsymbol{e}_{0}}(\tau)=E_{4}(\tau), \\
& \Theta_{2 E_{8}, \boldsymbol{e}_{0}}(\tau)=E_{4}(2 \tau), \\
& \Theta_{2 E_{8}, \boldsymbol{e}_{1}}(\tau)=\frac{1}{240}\left(E_{4}(\tau / 2)-E_{4}(\tau / 2+1 / 2)\right), \\
& \Theta_{2 E_{8}, \boldsymbol{e}_{2}}(\tau)=\frac{1}{15}\left(E_{4}(\tau)-E_{4}(2 \tau)\right) .
\end{aligned}
$$




\subsection{BPS invariants for $r \leq 3$}

Rank 1 The results from the periods for $h_{C, \mathbf{0}}^{\mathrm{pr}}(\tau)$ is (6.1)

$$
h_{C, \mathbf{0}}^{\mathrm{pr}}(\tau)=\frac{E_{4}(\tau)}{\eta(\tau)^{12}}
$$

This can easily be verified with the results for sheaves on surfaces. The result for $r=1$ and a complex, simply connected surface $S$ is [25]

$$
h_{1, c_{1}}(z, \tau ; S)=\frac{i}{\theta_{1}(2 z, \tau) \eta(\tau)^{b_{2}(S)-1}} .
$$

The dependence on $\mathcal{J}$ can be omitted for $r=1$ since all rank 1 sheaves are stable. If we specialize to $S=\mathbb{F}_{9}$, take the limit $w \rightarrow-1$, and sum over all $c_{1} \in E=H^{2}\left(\mathbb{F}_{9}, \mathbb{Z}\right) / \iota^{*} H^{2}(M, \mathbb{Z})$ one obtains $h_{C, \mathbf{0}}(\tau)=E_{4}(\tau) / \eta(\tau)^{12}$ in agreement with Equation (6.19).

Rank 2 The prediction by the periods for $r=2$ is given by $h_{2 C, \mathbf{0}}^{\mathrm{pr}}(\tau)$ in (6.1). This is a sum over all BPS invariants for $c_{1} \cdot \mathbf{a}_{i}=0, i=1,2$. In order to verify this result, it is useful to decompose $h_{2 C, \mathbf{0}}^{\mathrm{pr}}(\tau)$ according to the three conjugacy classes of $E / 2 E \in H_{2}\left(\mathbb{F}_{9}\right) /\left(\iota^{*} H_{2}(M, \mathbb{Z})\right)$. One obtains $h_{C, \mathbf{0}}^{\mathrm{pr}}(\tau)=$ $\sum_{i=0,1,2} m_{i} h_{2, \boldsymbol{e}_{i}}^{\mathrm{pr}}\left(\tau ; \mathbb{F}_{9}, \mathbf{a}_{1}\right) \Theta_{2 E_{8}, \boldsymbol{e}_{i}}(\tau)$ with [57]:

$$
\begin{aligned}
h_{2, \boldsymbol{e}_{0}}^{\mathrm{pr}}\left(\tau ; \mathbb{F}_{9}, \mathbf{a}_{1}\right)= & \frac{1}{24 \eta(\tau)^{24}}\left[E_{2}(\tau) \Theta_{2 E_{8}, \boldsymbol{e}_{0}}(\tau)+\left(\theta_{3}(\tau)^{4} \theta_{4}(\tau)^{4}-\frac{1}{8} \theta_{2}(\tau)^{8}\right)\right. \\
& \left.\times\left(\theta_{3}(\tau)^{4}+\theta_{4}(\tau)^{4}\right)\right]+\frac{1}{8} h_{1, \boldsymbol{e}_{0}}^{\mathrm{pr}}\left(2 \tau ; \mathbb{F}_{9}\right), \\
h_{2, \boldsymbol{e}_{1}}^{\mathrm{pr}}\left(\tau ; \mathbb{F}_{9}, \mathbf{a}_{1}\right)= & \frac{1}{24 \eta(\tau)^{24}}\left[E_{2}(\tau) \Theta_{2 E_{8}, \boldsymbol{e}_{1}}(\tau)-\frac{1}{8} E_{4}(\tau) \theta_{2}(\tau)^{4}\right], \\
h_{2, \boldsymbol{e}_{2}}^{\mathrm{pr}}\left(\tau ; \mathbb{F}_{9}, \mathbf{a}_{1}\right)= & \frac{1}{24 \eta(\tau)^{24}}\left[E_{2}(\tau) \Theta_{2 E_{8}, \boldsymbol{e}_{2}}(\tau)-\frac{1}{8} \theta_{2}(\tau)^{8}\left(\theta_{3}(\tau)^{4}+\theta_{4}(\tau)^{4}\right)\right] .
\end{aligned}
$$

In the following we will omit the argument " $F_{9}$ " in the from the generating functions.

Verification of the expressions (6.21) is much more elaborate then for $r=1$. We will use the approach of $[60,61,63]$. The main issues are the determination of the BPS invariants for a polarization close to the class $\mathbf{a}_{2}$ 
(a suitable polarization) and wall-crossing from the suitable polarization to $\mathcal{J}=-K_{9}=\mathbf{a}_{1}$. These issues are dealt with for the Hirzebruch surfaces [60, 61], and for $\mathbb{F}_{9}$ in [63]. The main difficulty for $\mathbb{F}_{9}$ compared to the Hirzebruch surfaces is that that the class $f$ and $K_{9}$ span the lattice $A$, which is related to $\Lambda_{1,9}$ by a non-trivial gluing with the lattice $D$.

Before turning to the explicit expressions, we briefly outline the computation; we refer for more details about the used techniques to [55]. The polarization $\mathcal{J}$ is parameterized by $\mathcal{J}_{m, n}=m \mathbf{a}_{1}+n \mathbf{a}_{2}$. In order to determine the BPS invariants for the suitable polarization $\mathcal{J}_{\varepsilon, 1}$, view $\mathbb{F}_{9}$ as the 8-point blow-up of the Hirzebruch surface $\mathbb{F}_{1}: \phi: \mathbb{F}_{9} \rightarrow \mathbb{F}_{1}$. We choose to perform this blow-up for the polarization $\mathcal{J}_{\mathbb{F}_{1}}=f$, with $f$ the fibre class of the Hirzebruch surface. The pull back of this class to $\mathbb{F}_{9}$ is $\phi^{*} f=\mathcal{J}_{0,1}$. The generating function of the BPS invariants for this choice takes a relatively simple form: it either vanishes or equals a product of eta and theta functions [55,61] depending on the Chern classes. This function represents the sheaves whose restriction to the rational curve $\mathbf{a}_{2}$ is semi-stable. The generating function $h_{r, c_{1}}\left(z, \tau ; \mathbb{F}_{9}, \mathcal{J}_{0,1}\right)$ is therefore this product formula multiplied by the factors due to blowing-up the 8 points. To obtain the BPS invariants from this function, one has to change $\mathcal{J}_{0,1}$ to $\mathcal{J}_{\varepsilon, 1}$ and subtract the contribution due to sheaves which became (Gieseker) unstable due to this change [55]. Consequently, we can determine the BPS invariants for any other choice of $\mathcal{J}$ by the wall-crossing formula [35,42,62]. In particular, we determine the invariants for $\mathcal{J}_{1,0}=-K_{9}$ and change to the basis $\mathbf{E}$ in order to compare with the expression from the periods.

We continue with determining the BPS invariants for $\mathcal{J}=\mathcal{J}_{0,1}$. The BPS invariants vanish for $c_{1} \cdot \mathbf{a}_{2}=1 \bmod 2$

$$
h_{2, c_{1}}\left(z, \tau ; \mathcal{J}_{\varepsilon, 1}\right)=0, \quad c_{1} \cdot \mathbf{a}_{2}=1 \quad \bmod 2 .
$$

Since BPS invariants depend on $c_{1} \bmod 2 \Lambda_{1,9}$, we distinguish further $c_{1} \cdot \mathbf{a}_{2}=0 \bmod 4$ and $c_{1} \cdot \mathbf{a}_{2}=2 \bmod 4$. For these cases, we continue as in [55] using the (extended) Harder-Narasimhan filtration. A sheaf $F$ which is unstable for $\mathcal{J}_{\varepsilon, 1}$ but semi-stable for $\mathcal{J}_{0,1}$, can be described as a $\mathrm{HN}$ filtration of length 2 whose quotients we denote by $E_{i}, i=1,2$. If we parameterize the first Chern class of $E_{2}$ by $\mathbf{k}=\left(\mathbf{k}_{A}, \mathbf{k}_{D}\right)$, then the discriminant $\Delta(F)$ is given by

$$
2 \Delta(F)=\Delta\left(E_{1}\right)+\Delta\left(E_{2}\right)-\frac{1}{4}\left(2 \mathbf{k}_{A}-\left.c_{1}\right|_{A}\right)^{2}-\frac{1}{4}\left(2 \mathbf{k}_{D}-\left.c_{1}\right|_{D}\right)^{2} .
$$


The choice of $\mathbf{k}_{D}$ does not have any effect on the stability of $F$ as long as $\mathcal{J}$ is spanned by $\mathcal{J}_{0,1}$ and $\mathcal{J}_{1,0}$. Therefore (6.23) shows that the sum over $\mathbf{k}_{D}$ gives rise to the theta functions $\Theta_{2 D_{8}, \mu}(\tau)$. The condition for semistability for $\mathcal{J}_{0,1}$ but unstable for $\mathcal{J}_{\varepsilon, 1}$ implies $\left(c_{1}\left(E_{1}\right)-c_{1}\left(E_{2}\right)\right) \cdot \mathbf{a}_{2}=0$. This combined with $c_{1} \cdot \mathbf{a}_{2}=0 \bmod 4$ gives for $c_{1}\left(E_{i}\right)=0 \bmod 2$, which shows that $c_{1}\left(E_{i}\right)=\boldsymbol{g}_{j} \bmod 2 \Lambda_{1,9}$ only for $j=0,2$. One obtains after a detailed analysis for $c_{1} \cdot \mathbf{a}_{2}=0 \bmod 4$

$$
\begin{aligned}
h_{2, c_{1}}\left(z, \tau ; \mathcal{J}_{\varepsilon, 1}\right)= & \frac{-i \eta(\tau)}{\theta_{1}(2 z, \tau)^{2} \theta_{1}(4 z, \tau)} \prod_{i=1}^{8} B_{2, \ell_{i}}(z, \tau) \\
+ & \left(\frac{w^{4\left\{\left(\frac{1}{2} \boldsymbol{g}_{0}-\frac{1}{4} c_{1}\right) \cdot \mathbf{a}_{1}\right\}}}{1-w^{4}}-\frac{1}{2} \delta_{0,\left\{\left(\frac{1}{2} \boldsymbol{g}_{0}-\frac{1}{4} c_{1}\right) \cdot \mathbf{a}_{1}\right\}}\right) \\
& \times \Theta_{2 D_{8}, c_{1}-2 \boldsymbol{g}_{0}}(\tau) h_{1, \mathbf{0}}(z, \tau)^{2} \\
+ & \left(\frac{w^{4\left\{\left(\frac{1}{2} \boldsymbol{g}_{2}-\frac{1}{4} c_{1}\right) \cdot \mathbf{a}_{1}\right\}}}{1-w^{4}}-\frac{1}{2} \delta_{0,\left\{\left(\frac{1}{2} \boldsymbol{g}_{2}-\frac{1}{4} c_{1}\right) \cdot \mathbf{a}_{1}\right\}}\right) \\
& \times \Theta_{2 D_{8}, c_{1}-2 \boldsymbol{g}_{2}}(\tau) h_{1, \mathbf{0}}(z, \tau)^{2},
\end{aligned}
$$

where $\{\lambda\}=\lambda-\lfloor\lambda\rfloor$ and $\ell_{i}=c_{1} \cdot \mathbf{c}_{i}$. The right-hand side on the first line correspond to the sheaves whose restriction to $\mathbf{a}_{2}$ are semi-stable. The functions $B_{2, \ell}(z, \tau)=\sum_{n \in \mathbb{Z}+\ell / 2} q^{n^{2}} w^{n} / \eta(\tau)^{2}$ are due to the blow-up formula $[27,43,55,62]$. The second and third line are the subtractions due to sheaves that are unstable for $\mathcal{J}_{\varepsilon, 1}$.

Similarly, one obtains for $c_{1} \cdot \mathbf{a}_{2}=2 \bmod 4$

$$
\begin{aligned}
h_{2, c_{1}}\left(z, \tau ; \mathcal{J}_{\varepsilon, 1}\right)= & \frac{-i \eta(\tau)}{\theta_{1}(2 z, \tau)^{2} \theta_{1}(4 z, \tau)} \prod_{i=1}^{8} B_{2, \ell_{i}}(z, \tau) \\
& +\left(\frac{w^{4\left\{\left(\frac{1}{2} \boldsymbol{g}_{1}-\frac{1}{4} c_{1}\right) \cdot \mathbf{a}_{1}\right\}}}{1-w^{4}}-\frac{1}{2} \delta_{0,\left\{\left(\frac{1}{2} \boldsymbol{g}_{1}-\frac{1}{4} c_{1}\right) \cdot \mathbf{a}_{1}\right\}}\right) \\
& \times \Theta_{2 D_{8}, c_{1}-2 \boldsymbol{g}_{1}(\tau) h_{1, \mathbf{0}}(z, \tau)^{2}} \\
& +\left(\frac{w^{4\left\{\left(\frac{1}{2} \boldsymbol{g}_{3}-\frac{1}{4} c_{1}\right) \cdot \mathbf{a}_{1}\right\}}}{1-w^{4}}-\frac{1}{2} \delta_{0,\left\{\left(\frac{1}{2} \boldsymbol{g}_{3}-\frac{1}{4} c_{1}\right) \cdot \mathbf{a}_{1}\right\}}\right) \\
& \times \Theta_{2 D_{8}, c_{1}-2 \boldsymbol{g}_{3}}(\tau) h_{1, \mathbf{0}}(z, \tau)^{2} .
\end{aligned}
$$

What remains is to change the polarization $\mathcal{J}$ from $\mathcal{J}_{\varepsilon, 1}$ to $\mathcal{J}_{1,0}$ and determine the change of the invariants using wall-crossing formulas. For 
Table 3: The Betti numbers $b_{n}$ (with $n \leq \operatorname{dim}_{\mathbb{C}} \mathcal{M}$ ) and Euler numbers $\chi$ of the moduli spaces of semi-stable sheaves on $\mathbb{F}_{9}$ with $r=2, c_{1}=\boldsymbol{e}_{i}$, and $1 \leq c_{2} \leq 4$ for $\mathcal{J}=\mathcal{J}_{1,0}$.

\begin{tabular}{rrrrrrrrrrrl}
\hline$c_{1}$ & $c_{2}$ & $b_{0}$ & $b_{2}$ & $b_{4}$ & $b_{6}$ & $b_{8}$ & $b_{10}$ & $b_{12}$ & $b_{14}$ & $b_{16}$ & \multicolumn{1}{c}{$\chi$} \\
\hline $\boldsymbol{e}_{0}$ & 2 & 1 & 10 & 55 & & & & & & & 132 \\
& 3 & 1 & 11 & 76 & 396 & 1356 & & & & & 3680 \\
& 4 & 1 & 11 & 78 & 428 & 1969 & 7449 & 20124 & & & 60120 \\
& 5 & 1 & 11 & 78 & 430 & 2012 & 8316 & 30506 & 95498 & 221132 & 715968 \\
$\boldsymbol{e}_{1}$ & 1 & 1 & 9 & & & & & & & & 20 \\
& 2 & 1 & 11 & 75 & 309 & & & & & & 792 \\
& 3 & 1 & 11 & 78 & 426 & 1843 & 5525 & & & & 15768 \\
& 4 & 1 & 11 & 78 & 430 & 2010 & 8150 & 27777 & 68967 & & 214848 \\
$\boldsymbol{e}_{2}$ & 1 & 1 & & & & & & & & & 2 \\
& 2 & 1 & 11 & 60 & & & & & & & 144 \\
& 3 & 1 & 11 & 78 & 404 & 1386 & & & & & 3760 \\
& 4 & 1 & 11 & 78 & 430 & 1981 & 7495 & 20244 & & & 60480 \\
\hline
\end{tabular}

$\mathcal{J}=(m, n, \mathbf{0}) \in A \oplus D$, we obtain the following expression:

$$
\begin{aligned}
h_{2, c_{1}}\left(z, \tau ; \mathcal{J}_{m, n}\right)= & \frac{-i \eta(\tau)}{\theta_{1}(2 z, \tau)^{2} \theta_{1}(4 z, \tau)} \prod_{i=1}^{8} B_{2, \ell_{i}}(z, \tau) \\
& +\sum_{j=0, \ldots, 3} h_{2, c_{1}-2 \boldsymbol{g}_{j}}^{A}\left(z, \tau ; \mathcal{J}_{m, n}\right) \Theta_{2 D, c_{1}-2 \boldsymbol{g}_{j}}(\tau),
\end{aligned}
$$

with

$$
\begin{aligned}
h_{2, c_{1}}^{A}\left(z, \tau ; \mathcal{J}_{m, n}\right)= & h_{2, c_{1}}^{A}\left(z, \tau ; \mathcal{J}_{\varepsilon, 1}\right)+\frac{1}{2} \sum_{\left(a_{1}, a_{2}\right) \in A+c_{1}} \\
& \frac{1}{2}\left(\operatorname{sgn}\left(a_{1} n+a_{2} m\right)-\operatorname{sgn}\left(a_{1}+a_{2} \varepsilon\right)\right) \\
& \times\left(w^{4 a_{2}}-w^{-4 a_{2}}\right) q^{-4 a_{1} a_{2}} h_{1, \mathbf{0}}(z, \tau)^{2} .
\end{aligned}
$$

The functions $h_{2, c_{1}}^{A}\left(z, \tau ; \mathcal{J}_{\varepsilon, 1}\right)$ are rational functions in $w$ multiplied by $h_{1,0}(z, \tau)^{2}$, which can easily be read off from Equation (6.24). For $\mathcal{J}=\mathcal{J}_{1,0}$ the functions can be expressed in terms of modular functions.

Table 3 presents the BPS invariants for $\mathcal{J}=\mathcal{J}_{1,0}$. As expected, the Euler numbers are indeed in agreement with the predictions (6.21). One can also verify that for increasing $c_{2}$, the Betti numbers asymptote to those of $r=1$ or equivalently the Hilbert scheme of points of $\mathbb{F}_{9}$. 
We define the functions $h_{2, c_{1}}^{A}(z, \tau):=h_{2, c_{1}}^{A}\left(z, \tau ; \mathcal{J}_{1,0}\right)$, which only depend on $\left.c_{1}\right|_{A}=\alpha_{1} \mathbf{a}_{1}+\alpha_{2} \mathbf{a}_{2}$ with $\alpha_{1}, \alpha_{2} \in 0, \frac{1}{2}, 1, \frac{3}{2}$. One finds for $\alpha_{2}=0 \bmod 4$

$$
\frac{h_{2, c_{1}}^{A}(z, \tau)}{h_{1,0}(z, \tau)^{2}}=-\frac{1}{8} \frac{1}{2 \pi \mathrm{i}} \frac{\partial}{\partial z} \ln \left(\theta_{1}\left(4 \tau, 4 z+2 \alpha_{1}\right) \theta_{1}\left(4 \tau, 4 z-2 \alpha_{1}\right)\right)
$$

and for $\alpha_{2} \neq 0 \bmod 4$ using (C.3)

$$
\begin{aligned}
\frac{h_{2, c_{1}}^{A}(z, \tau)}{h_{1,0}(z, \tau)^{2}}= & \frac{i}{2} \frac{q^{-\alpha_{1} \alpha_{2}} \eta(4 \tau)^{3}}{\theta_{1}\left(4 \tau, 2 \alpha_{2} \tau\right)}\left(\frac{w^{-2 \alpha_{2}} \theta_{1}\left(4 \tau, 4 z+2\left(\alpha_{1}-\alpha_{2}\right) \tau\right)}{\theta_{1}\left(4 \tau, 4 z+2 \alpha_{1} \tau\right)}\right. \\
& \left.-\frac{w^{2 \alpha_{2}} \theta_{1}\left(4 \tau,-4 z+2\left(\alpha_{1}-\alpha_{2}\right) \tau\right)}{\theta_{1}\left(4 \tau, 4 z+2 \alpha_{1} \tau\right)}\right)
\end{aligned}
$$

To prove the agreement of the Euler numbers with the periods, we specialize to $w=-1$. Let $D_{k}=\frac{1}{2 \pi \mathrm{i}} \frac{\partial}{\partial \tau}-\frac{k}{12} E_{2}(\tau)$ be the differential operator which maps weight $k$ modular forms to modular forms of weight $k+2$. Then one can write $h_{2, c_{1}}\left(\tau ; \mathcal{J}_{1,0}\right)$ as

$$
\begin{aligned}
h_{2, c_{1}}\left(\tau ; \mathcal{J}_{1,0}\right)= & \frac{1}{\eta(\tau)^{24}}\left(\frac{1}{2} \delta_{c_{1} \cdot \mathbf{a}_{2}, 0} D_{4}\left(\theta_{3}(2 \tau)^{m} \theta_{2}(2 \tau)^{8-m}\right)\right. \\
& \left.+\sum_{i=0, \ldots, 3} f_{c_{1}-2 \boldsymbol{g}_{j}}^{A}(\tau) \Theta_{2 D, c_{1}-2 \boldsymbol{g}_{j}}(\tau)\right),
\end{aligned}
$$

with

$$
\begin{aligned}
f_{0,0}^{A}(\tau) & =\frac{1}{8} \theta_{3}(2 \tau)^{4}+\frac{1}{24} E_{2}(\tau), \\
f_{-\frac{1}{2}, \frac{1}{2}}^{A}(\tau) & =\frac{1}{2} \theta_{2}(2 \tau) \theta_{3}(2 \tau)^{3}, \\
f_{\frac{1}{2}, \frac{1}{2}}^{A}(\tau) & =\frac{1}{2} \theta_{2}(2 \tau)^{3} \theta_{3}(2 \tau), \\
f_{1,0}^{A}(\tau) & =\frac{1}{12} \theta_{2}(2 \tau)^{4}-\frac{1}{24} \theta_{3}(2 \tau)^{4}+\frac{1}{24} E_{2}(\tau), \\
f_{0,1}^{A}(\tau) & =\frac{1}{24} \theta_{2}(2 \tau)^{4}-\frac{1}{12} \theta_{3}(2 \tau)^{4}, \\
f_{1,1}^{A}(\tau) & =-\frac{1}{8} \theta_{2}(2 \tau)^{4} .
\end{aligned}
$$

If $\left.c_{1}\right|_{B}=0$, this reproduces the functions in $[57,63]$ depending on whether the classes in lattice $E$ are even or odd.

Modularity Electric-magnetic duality of $\mathcal{N}=4 U(r)$ Yang-Mills theory implies modular properties for its partition function [59]. Determination 
of the modular properties gives therefore insight about the realization of electric-magnetic duality at the quantum level.

The expression in Equation (6.26) does not transform as a modular form for generic choices of $\mathcal{J}$. However, using the theory of indefinite theta functions [64], the functions can be completed to a function $\widehat{h}_{2, c_{1}}(z, \tau ; \mathcal{J})$ by addition of a non-holomorphic term, such that $\widehat{h}_{2, c_{1}}(z, \tau ; \mathcal{J})$ does transform as a modular form [53]. Interestingly, Equation $(6.29)$ shows that $h_{2, c_{1}}(z, \tau ; \mathcal{J})$ becomes a quasi-modular form for $\lim _{\mathcal{J} \rightarrow \mathcal{J}_{1,0}} \widehat{h}_{2, c_{1}}(z, \tau ; \mathcal{J})$, i.e., it can be expressed in terms of modular forms and Eisenstein series of weight 2 . In some cases it becomes even a true modular form. This is due to the special form of $\mathcal{Q}_{A}$.

The transition from mock modular to quasi-modular can be made precise. Owing to the gluing vectors, the function $f_{2, c_{1}}(z, \tau ; \mathcal{J})=h_{2, c_{1}}(z, \tau ; \mathcal{J}) /$ $h_{1, c_{1}}(z, \tau)^{2}$ takes the form

$$
\begin{aligned}
f_{2, c_{1}}\left(z, \tau ; \mathcal{J}_{m, n}\right)= & \sum_{\mu} f_{2,\left(c_{1}-2 \mu\right)_{A}}^{A}\left(z, \tau ; \mathcal{J}_{m, n}\right) \Theta_{2 D,\left(2 \mu-c_{1}\right)_{D}}(\tau) \\
& +\delta_{c_{1} \cdot \mathbf{a}_{2}, 0} \frac{i \eta(\tau)^{3}}{\theta_{1}(\tau, 4 z)} \theta_{3}(2 \tau, 2 z)^{k} \theta_{2}(2 \tau, 2 z)^{8-k}
\end{aligned}
$$

where $k$ is the number of $c_{1} \cdot \mathbf{c}_{i}=1 \bmod 2$ for $1 \leq i \leq 8$. The completed generating function $\widehat{f}_{2, c_{1}}(z, \tau ; \mathcal{J})$ is a slight generalization of the equations in Section 3.2 in [53]:

$$
\begin{aligned}
\widehat{f}_{2, c_{1}}(\tau ; \mathcal{J})= & f_{2, c_{1}}(\tau ; \mathcal{J})+\sum_{\substack{\mathbf{c} \in-c_{1} \\
+H^{2}\left(\Sigma_{9}, 2 \mathbb{Z}\right)}}\left(\frac{K_{9} \cdot \mathcal{J}}{4 \pi \sqrt{\mathcal{J}^{2} \tau_{2}}} \mathrm{e}^{-\pi \tau_{2} \mathbf{c}_{+}^{2}}\right. \\
& \left.-\frac{1}{4} K_{9} \cdot \mathbf{c} \operatorname{sgn}(\mathbf{c} \cdot \mathcal{J}) \beta_{\frac{1}{2}}\left(\mathbf{c}_{+}^{2} \tau_{2}\right)\right)(-1)^{K_{9} \cdot \mathbf{c}} q^{-\mathbf{c}^{2} / 4}
\end{aligned}
$$

where $\tau_{2}=\operatorname{Im}(\tau)$ and $\beta_{\nu}(x)=\int_{x}^{\infty} u^{-\nu} \mathrm{e}^{-\pi u} d u$. We parameterize $\mathcal{J}$ by $b_{1}+$ $v b_{2}$ and carefully study the limit $v \rightarrow 0$ (this corresponds to the limit $R \rightarrow$ $\infty$ in [57]). In this limit, $\mathcal{J}$ approaches $-K_{9}$. Moreover, $\mathcal{J} \cdot K_{9}=-v$ and $\mathcal{J}^{2}=v(2-v)$. If one parameterizes $\mathbf{c}$ by $\left(n_{0}, n_{1}, \mathbf{c}_{\perp}\right)$, only terms with $n_{1}=0$ contribute to the sum in the limit $v \rightarrow 0$. Therefore the term with $\beta_{\frac{1}{2}}\left(\mathbf{c}_{+}^{2} \tau_{2}\right)$ does not contribute to the anomaly. After a Poisson resummation on $n_{0}$, one finds that the limit is finite and given by

$$
\widehat{f_{2, c_{1}}}\left(\tau ; \mathcal{J}_{1,0}\right)=f_{2, c_{1}}\left(\tau ; \mathcal{J}_{1,0}\right)-\frac{\delta_{c_{1} \cdot \mathbf{a}_{1}, 0}}{8 \pi \tau_{2}} \sum_{\substack{\mathbf{c} \in-c_{1} \mid E \\+2 E}} q^{-\mathbf{c}_{\perp}^{2} / 4}
$$


This is in good agreement with Equation (6.21) if $c_{1} \cdot \mathbf{a}_{1}=0$. The lattice sum over $\mathbf{c}$ gives precisely the theta functions $\Theta_{2 E_{8}, \mathbf{e}_{i}}(\tau)$. Recalling the modular completion of the weight 2 Eisenstein series: $\widehat{E}_{2}(\tau)=E_{2}(\tau)-\frac{3}{\pi \tau_{2}}$, we see that the non-holomorphic term implies that in the holomorphic part of $\widehat{f}_{2, c_{1}}\left(\tau ; \mathcal{J}_{1,0}\right), E_{2}(\tau)$ is multiplied by the $\Theta_{2 E_{8}, \mathbf{e}_{i}}(\tau) / 24$ as in Equation (6.21). We have thus verified that the non-holomorphic dependence of $D 4$-brane partition functions is indeed consistent with (6.21) and therefore with (3.9) for topological strings as implied by T-duality. Note that for $c_{1} \cdot \mathbf{a}_{1}=1$ $\bmod 2$, the non-holomorphic dependence of $f_{2, c_{1}}(\tau ; \mathcal{J})$ vanishes in the limit $\mathcal{J} \rightarrow \mathcal{J}_{1,0}$, in agreement with (6.29).

Rank 3 Similarly as for $r=2$, [57] also decomposes $h_{3 C, \mathbf{0}}^{\mathrm{pr}}(\tau)$ into different Weyl orbits. We will restrict in the following to the $\boldsymbol{e}_{0}=\mathbf{0}$ orbit in $E / 3 E$ since the expressions become rather lengthy. In order to present $h_{3, e_{0}}^{\mathrm{pr}}\left(\tau ; \mathbf{a}_{1}\right)$, define

$$
b_{3, \ell}(\tau)=\sum_{m, n \in \mathbb{Z}+\ell / 3} q^{m^{2}+n^{2}+m n}
$$

Then $h_{3, e_{0}}^{\mathrm{pr}}\left(\tau ; \mathbf{a}_{1}\right)$ is given by $[57]$

$$
\begin{aligned}
h_{3, e_{0}}^{\mathrm{pr}}\left(\tau ; \mathbf{a}_{1}\right)= & \frac{1}{2592 \eta^{36}}\left[\left(51 b_{3,0}^{12}-184 b_{3,0}^{9} b_{3,1}^{3}+336 b_{3,0}^{6} b_{3,1}^{6}\right.\right. \\
& \left.+288 b_{3,0}^{3} b_{3,1}^{9}+32 b_{3,1}^{12}\right) \\
& +E_{2} b_{3,0}\left(36 b_{3,0}^{9}-112 b_{3,0}^{6} b_{3,1}^{3}+32 b_{3,0}^{6} b_{3,1}^{3}-64 b_{3,1}^{9}\right) \\
& \left.+E_{2}^{2} b_{3,0}^{2}\left(9 b_{3,0}^{6}-16 b_{3,0}^{3} b_{3,1}^{3}+16 b_{3,0}^{6}\right)\right] .
\end{aligned}
$$

In order to verify this expression, we extend the analysis for $r=2$ to $r=3$. For $c_{1} \cdot \mathbf{a}_{2}= \pm 1 \bmod 3$, the BPS invariants vanish for a suitable polarization

$$
h_{3, c_{1}}\left(z, \tau ; \mathcal{J}_{\varepsilon, 1}\right)=0 \text {. }
$$

The HN-filtrations for the sheaves which are unstable for $\mathcal{J}_{\varepsilon, 1}$ but semistable for $\mathcal{J}_{0,1}$ have length 2 or 3 . From those of length 2 , one obtains rational functions in $w$ multiplied by $h_{1,0}(z, \tau) h_{2, \boldsymbol{\mu}}(z, \tau) \Theta_{2 D_{8}, \boldsymbol{\mu}}(\tau)$, with $\boldsymbol{\mu}=\mathbf{0}, \mathbf{a}_{2}$, $\boldsymbol{d}_{i}$ and $\boldsymbol{d}_{i}+\mathbf{a}_{2}$. The theta function arising from the sum over the $D_{8}$ lattice is more involved for filtrations of length 3 . Instead of a direct sum, a "twisted" 
sum of $2 D_{8}$-lattices appears; we will denote this lattice by $D_{8}^{\mathrm{t}}$

$$
\begin{aligned}
\Theta_{2 D_{8}^{\mathbf{t}} ; \boldsymbol{\mu}_{1}, \boldsymbol{\mu}_{2}}(\tau) & =\sum_{\mathbf{k}_{i} \in D_{8}+\boldsymbol{\mu}_{i}, i=1,2} q^{\mathbf{k}_{1}^{2}+\mathbf{k}_{1} \cdot \mathbf{k}_{2}+\mathbf{k}_{2}^{2}} \\
& =\sum_{i} m_{i} \Theta_{2 D_{8}, \boldsymbol{\mu}_{1}+\boldsymbol{\mu}_{2}+\boldsymbol{d}_{i}}(\tau) \Theta_{2 D_{8}, \boldsymbol{\mu}_{1}-\boldsymbol{\mu}_{2}+\boldsymbol{d}_{i}}(3 \tau)
\end{aligned}
$$

where $m_{i}$ are the multiplicities of the theta characteristics $\boldsymbol{\mu}_{1}+\boldsymbol{\mu}_{2}+\boldsymbol{d}_{i}$, thus for $\boldsymbol{\mu}_{1}+\boldsymbol{\mu}_{2} \in D, i=1, \ldots, 6$, and for $\boldsymbol{\mu}_{1}+\boldsymbol{\mu}_{2} \in D / 2, i=1, \ldots, 4$. For numerical computations the second line is considerably faster than the first line. We obtain after a careful analysis

$$
\begin{aligned}
& h_{3,0}\left(z, \tau ; \mathcal{J}_{\varepsilon, 1}\right) \\
&=\frac{i \eta(\tau)^{3}}{\theta_{1}(2 z, \tau)^{2} \theta_{1}(4 z, \tau)^{2} \theta_{1}(6 z, \tau)} B_{3,0}(z, \tau)^{8} \\
& \quad+2\left(\frac{1}{1-w^{12}}-\frac{1}{2}\right) h_{1,0}(z, \tau) \sum_{i=0,3} h_{2,\left(0,0, \boldsymbol{d}_{i}\right)}\left(z, \tau ; \mathcal{J}_{\varepsilon, 1}\right) \Theta_{2 D_{8}, \boldsymbol{d}_{i}}(3 \tau) \\
&+2\left(\frac{w^{6}}{1-w^{12}}\right) h_{1,0}(z, \tau) \sum_{i=0,3} h_{2,\left(0,1, \boldsymbol{d}_{i}\right)}\left(z, \tau ; \mathcal{J}_{\varepsilon, 1}\right) \Theta_{2 D_{8}, \boldsymbol{d}_{i}}(3 \tau) \\
&+2\left(\frac{1}{1-w^{6}}-\frac{1}{2}\right) h_{1,0}(z, \tau) \sum_{i=1,2,4,5} m_{0, i} h_{2,\left(0,0, \boldsymbol{d}_{i}\right)}\left(z, \tau ; \mathcal{J}_{\varepsilon, 1}\right) \Theta_{2 D_{8}, \boldsymbol{d}_{i}}(3 \tau) \\
&+2\left(\frac{w^{3}}{1-w^{6}}\right) h_{1,0}(z, \tau) \sum_{i=0,1,2,4} m_{2, i} h_{2, \boldsymbol{g}_{2}+\boldsymbol{d}_{i}}\left(z, \tau ; \mathcal{J}_{\varepsilon, 1}\right) \Theta_{2 D_{8}, \boldsymbol{g}_{2}+\boldsymbol{d}_{i}}(3 \tau) \\
& \\
& \quad-\left(\frac{1+w^{12}}{\left(1-w^{8}\right)\left(1-w^{12}\right)}-\frac{1}{1-w^{12}}+\frac{1}{6}\right) h_{1,0}(z, \tau)^{3} \Theta_{2 D_{8}^{\mathrm{t}} ; 0,0}(\tau) \\
&-2\left(\frac{w^{6}}{\left(1-w^{4}\right)\left(1-w^{12}\right)}-\frac{w^{6}}{1-w^{12}}\right) h_{1,0}(z, \tau)^{3} \Theta_{2 D_{8}^{\mathrm{t}} ; \boldsymbol{g}_{2}, 0}(\tau) \\
&-\left(\frac{w^{4}+w^{16}}{\left(1-w^{8}\right)\left(1-w^{12}\right)}\right) h_{1,0}(z, \tau)^{3} \Theta_{2 D_{8}^{\mathrm{t}} ; 0,0}(\tau)
\end{aligned}
$$

The functions due to the blowing-up of eight points are now given by $B_{3, k}(z, \tau)=\sum_{m, n \in \mathbb{Z}+k / 3} q^{m^{2}+n^{2}+m n} w^{4 m+2 n} / \eta(\tau)^{3}$. We have used in (6.38) that $h_{2, c_{1}}\left(z, \tau ; \mathcal{J}_{m, n}\right)$ only depends on the conjugacy class of $c_{1}$ in $D / 2 D$, and moreover that $h_{2, c_{1}}\left(z, \tau ; \mathcal{J}_{m, n}\right)=h_{2, c_{1}^{\prime}}\left(z, \tau ; \mathcal{J}_{m, n}\right)$ if $c_{1}=\left(0,0, \boldsymbol{d}_{i}\right)$ and $c_{1}^{\prime}=\left(0,1, \boldsymbol{d}_{i}\right)$ for $i=1,2,4,5$ (but not for $\left.i=0,3\right)$ and $c_{1}=\left(0,0, \boldsymbol{d}_{i}\right)+\boldsymbol{g}_{2}$ and $c_{1}^{\prime}=\left(0,1, \boldsymbol{d}_{i}\right)+\boldsymbol{g}_{2}$. 
Table 4: The Betti numbers $b_{n}$ (with $n \leq \operatorname{dim}_{\mathbb{C}} \mathcal{M}$ ) and the Euler number $\chi$ of the moduli spaces of semi-stable sheaves on $\mathbb{F}_{9}$ with $r=3, c_{1}=0$, and $3 \leq c_{2} \leq 5$ for $\mathcal{J}=\mathcal{J}_{1, \varepsilon}$.

\begin{tabular}{lrrrrrrr}
\hline$c_{2}$ & \multicolumn{1}{c}{$b_{0}$} & \multicolumn{1}{c}{$b_{2}$} & \multicolumn{1}{c}{$b_{4}$} & \multicolumn{1}{c}{$b_{6}$} & \multicolumn{1}{c}{$b_{8}$} & \multicolumn{1}{c}{$b_{10}$} & $b_{12}$ \\
\hline 3 & 1 & 10 & 65 & 320 & 1025 & 1226 & \\
4 & 1 & 11 & 77 & 417 & 1902 & 7372 & 23962 \\
5 & 1 & 11 & 78 & 429 & 2002 & 8260 & 30710 \\
\hline$c_{2}$ & \multicolumn{1}{c}{$b_{14}$} & $b_{16}$ & $b_{18}$ & $b_{20}$ & $b_{22}$ & $\chi$ & \\
\hline 3 & & & & & & 4068 & \\
4 & 57452 & 68847 & & & & 251235 & \\
5 & 103867 & 316586 & 836221 & 1706023 & 2029416 & 8037792 & \\
\hline
\end{tabular}

Having determined $h_{3,0}\left(z, \tau ; \mathcal{J}_{\varepsilon, 1}\right)$, what rests is to perform the wallcrossing from $\mathcal{J}_{\varepsilon, 1}$ to $\mathcal{J}_{1,0}$. To this end, we define

$$
\begin{aligned}
h_{3, c_{1}}^{A}(z, \tau ; \mathcal{J})= & \sum_{\substack{\mathbf{a}=\left.c_{1}\right|_{A} \\
\bmod 2 A}} \frac{1}{2}\left(\operatorname{sgn}\left(a_{1} n+a_{2} m\right)-\operatorname{sgn}\left(a_{1}+a_{2} \varepsilon\right)\right) \\
& \left(w^{6 a_{2}}-w^{-6 a_{2}}\right) q^{-3 a_{1} a_{2}} h_{2,\left(\mathbf{a},\left.c_{1}\right|_{D}\right)}\left(z, \tau ; \mathcal{J}_{\left|a_{1}\right|,\left|a_{2}\right|}\right) h_{1,0}(z, \tau),
\end{aligned}
$$

with $\mathbf{a}=\left(a_{1}, a_{2}\right)$. Then $h_{3,0}(z, \tau ; \mathcal{J})$ is given by $[50,51]$

$$
\begin{aligned}
h_{3,0}(z, \tau ; \mathcal{J})= & h_{3,0}\left(z, \tau ; \mathcal{J}_{\mathcal{\varepsilon}, 1}\right) \\
& +\sum_{\mathbf{a} \in 2 A / A} m_{i, j} h_{3, \mathbf{a}+\boldsymbol{g}_{i}+\boldsymbol{d}_{j}}^{A}(z, \tau ; \mathcal{J}) \Theta_{2 D_{8}, \boldsymbol{g}_{i}+\boldsymbol{d}_{j}}(3 \tau) .
\end{aligned}
$$

The Betti numbers for $\mathcal{J}=\mathcal{J}_{1,0}$ and small $c_{2}$ are presented in table 4 , and indeed agree with the Euler numbers computed from the periods.

One might wonder how to derive the modular properties $h_{3,0}(z, \tau ; \mathcal{J})$. The completion takes in general a very complicated form due to the quadratic condition on the lattice points [51]. One can show however that for $\mathcal{J}=\mathcal{J}_{1,0}$ the quadratic condition disappears from the generating function due to a special symmetry of the lattice $A$, and therefore one again obtains quasimodular forms at this point. ${ }^{6}$

\footnotetext{
${ }^{6}$ We thank S. Zwegers for providing this argument.
} 


\section{Appendix A. Toric data for the elliptic hypersurfaces}

Here, we collect the toric data necessary to treat all models discussed. We list the Mori cones in the star triangulation for the bases of model 8-15 of figure 1

$$
\begin{aligned}
& \begin{array}{c|rrrr|rrrrr|}
\Delta_{B} & \multicolumn{7}{|c}{8(4)} & \multicolumn{7}{c}{9(4)} \\
\nu_{i}^{B} & l^{(1)} & l^{(2)} & l^{(3)} & l^{(4)} & l^{(1)} & l^{(1)} & l^{(2)} & l^{(1)} & l^{(2)} \\
z & 0 & -1 & 0 & -1 & -1 & -1 & 0 & -1 & -1 \\
1 & 0 & 0 & 0 & 1 & -1 & 1 & 0 & 0 & 0 \\
2 & 1 & 0 & 0 & 0 & 1 & -1 & 1 & 0 & 0 \\
3 & -2 & 1 & 0 & 0 & 0 & 1 & -2 & 1 & 0 \\
4 & 1 & -1 & 1 & 0 & 0 & 0 & 1 & -1 & 1 \\
5 & 0 & 1 & -2 & 1 & 0 & 0 & 0 & 1 & -1 \\
6 & 0 & 0 & 1 & -1 & 1 & 0 & 0 & 0 & 1 \\
e x & & & & & & & & &
\end{array} \\
& 10(4) \quad 11(5) \\
& \begin{array}{rrrr|rrrrrr|}
l^{(1)} & l^{(2)} & l^{(3)} & l^{(4)} & l^{(1)} & l^{(2)} & l^{(3)} & l^{(4)} & l^{(5)} & l^{(6)} \\
0 & -1 & 0 & 0 & 0 & -1 & 0 & 0 & -1 & -1 \\
1 & 0 & 0 & 0 & 1 & 0 & 0 & 0 & 0 & 1 \\
-2 & 1 & 0 & 0 & -2 & 1 & 0 & 0 & 0 & 0 \\
1 & -1 & 1 & 0 & 1 & -1 & 1 & 0 & 0 & 0 \\
0 & 1 & -2 & 1 & 0 & 1 & -2 & 1 & 0 & 0 \\
0 & 0 & 1 & -2 & 0 & 0 & 1 & -2 & 1 & 0 \\
0 & 0 & 0 & 1 & 0 & 0 & 0 & 1 & -1 & 1 \\
& & & & 0 & 0 & 0 & 0 & 1 & -1
\end{array}
\end{aligned}
$$

\begin{tabular}{c|rrrrrrr|rrrrrrr|}
$\Delta_{B}$ & \multicolumn{10}{|c}{$12(5)$} & \multicolumn{10}{c}{$13(6)$} \\
$\nu_{i}^{B}$ & $l^{(1)}$ & $l^{(2)}$ & $l^{(3)}$ & $l^{(4)}$ & $l^{(5)}$ & $l^{(6)}$ & $l^{(7)}$ & $l^{(1)}$ & $l^{(2)}$ & $l^{(3)}$ & $l^{(4)}$ & $l^{(5)}$ & $l^{(6)}$ & $l^{(7)}$ \\
$z$ & -1 & -1 & -1 & 0 & -1 & 0 & -1 & 0 & -1 & 0 & 0 & 0 & -1 & 0 \\
1 & 1 & 1 & 0 & 0 & 0 & 0 & 0 & -2 & 1 & 0 & 0 & 0 & 0 & 0 \\
2 & 0 & -1 & 1 & 0 & 0 & 0 & 0 & 1 & -1 & 1 & 0 & 0 & 0 & 0 \\
3 & 0 & 1 & -1 & 1 & 0 & 0 & 0 & 0 & 1 & -2 & 1 & 0 & 0 & 0 \\
4 & 0 & 0 & 1 & -2 & 1 & 0 & 0 & 0 & 0 & 1 & -2 & 1 & 0 & 0 \\
5 & 0 & 0 & 0 & 1 & -1 & 1 & 0 & 0 & 0 & 0 & 1 & -2 & 1 & 0 \\
6 & 1 & 0 & 0 & 0 & 1 & -2 & -2 & 0 & 0 & 0 & 0 & 1 & -1 & 1 \\
7 & -1 & 0 & 0 & 0 & 0 & 1 & 1 & 0 & 0 & 0 & 0 & 0 & 1 & -2 \\
8 & & & & & & & & 1 & 0 & 0 & 0 & 0 & 0 & 1 \\
$e x$ & & & & & 29 & & & & & & & 20 &
\end{tabular}




$$
\begin{array}{rrrrrrrr}
\multicolumn{8}{c}{14(6)} \\
l^{(1)} & l^{(2)} & l^{(3)} & l^{(4)} & l^{(5)} & l^{(6)} & l^{(7)} & l^{(8)} \\
-1 & 0 & -1 & 0 & 0 & -1 & 0 & -1 \\
-1 & 1 & 0 & 0 & 0 & 0 & 0 & 1 \\
1 & -2 & 1 & 0 & 0 & 0 & 0 & 0 \\
0 & 1 & -1 & 1 & 0 & 0 & 0 & 0 \\
0 & 0 & 1 & -2 & 1 & 0 & 0 & 0 \\
0 & 0 & 0 & 1 & -1 & 1 & 0 & 0 \\
0 & 0 & 0 & 0 & 1 & -1 & 1 & 0 \\
0 & 0 & 0 & 0 & 0 & 1 & -2 & 1 \\
1 & 0 & 0 & 0 & 0 & 0 & 1 & -1 \\
& & & & & & 43 &
\end{array}
$$$$
\begin{array}{c|rrrrrrrr|}
\Delta_{B} & \multicolumn{1}{|c}{15(5)} \\
\nu_{i}^{B} & l^{(1)} & l^{(2)} & l^{(3)} & l^{(4)} & l^{(5)} & l^{(6)} & l^{(7)} & l^{(8)} \\
z & 0 & -1 & 0 & -1 & 0 & -1 & 0 & -1 \\
1 & -2 & 1 & 0 & 0 & 0 & 0 & 0 & 1 \\
2 & 1 & -1 & 1 & 0 & 0 & 0 & 0 & 0 \\
3 & 0 & 1 & -2 & 1 & 0 & 0 & 0 & 0 \\
4 & 0 & 0 & 1 & -1 & 1 & 0 & 0 & 0 \\
5 & 0 & 0 & 0 & 1 & -2 & 1 & 0 & 0 \\
6 & 0 & 0 & 0 & 0 & 1 & -1 & 1 & 0 \\
7 & 0 & 0 & 0 & 0 & 0 & 1 & -2 & 1 \\
8 & 1 & 0 & 0 & 0 & 0 & 0 & 1 & -1 \\
9 & & & & & & & & \\
e x & & & & & & & & 53
\end{array}
$$

$$
\begin{array}{rrrrrrrrr}
l^{(1)} & l^{(2)} & l^{(3)} & l^{(4)} & l^{(5)} & l^{(6)} & l^{(7)} & l^{(8)} & l^{(9)} \\
0 & 0 & -1 & 0 & 0 & -1 & 0 & 0 & -1 \\
-2 & 1 & 0 & 0 & 0 & 0 & 0 & 0 & 1 \\
1 & -2 & 1 & 0 & 0 & 0 & 0 & 0 & 0 \\
0 & 1 & -1 & 1 & 0 & 0 & 0 & 0 & 0 \\
0 & 0 & 1 & -2 & 1 & 0 & 0 & 0 & 0 \\
0 & 0 & 0 & 1 & -2 & 1 & 0 & 0 & 0 \\
0 & 0 & 0 & 0 & 1 & -1 & 1 & 0 & 0 \\
0 & 0 & 0 & 0 & 0 & 1 & -2 & 1 & 0 \\
0 & 0 & 0 & 0 & 0 & 0 & 1 & -2 & 1 \\
1 & 0 & 0 & 0 & 0 & 0 & 0 & 1 & -1
\end{array}
$$



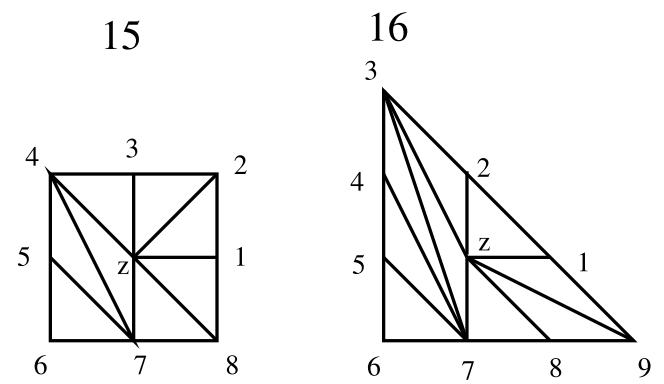

Figure 4: Non-star triangulations of the basis of model 15 and 16, which lead to simplicial Kähler cone for the Calabi-Yau space.

The simplicial Mori cone for the model 15 and 16 occur, e.g., for the triangulation depicted in figure 4 . For the model 15, the Mori cone reads

(A.1)

$$
\begin{array}{ll}
l^{(e)}=(-6,0,0,0,0,1,-1,1,0,0,2,3), & l^{(1)}=(0,-2,1,0,0,0,0,0,1,0,0,0) \\
l^{(2)}=(0,1,-1,1,0,0,0,0,0,-1,0,0), & l^{(3)}=(0,0,1,-2,1,0,0,0,0,0,0,0), \\
l^{(4)}=(0,0,0,1,-1,1,0,0,0,-1,0,0), & l^{(5)}=(0,0,0,0,0,-1,0,1,-1,1,0,0), \\
l^{(6)}=(0,0,0,0,0,0,1,-2,1,0,0,0) . &
\end{array}
$$

This yields the intersection numbers

$$
\begin{aligned}
(\mathrm{A} .2) & \\
\mathcal{R}= & 4 J_{\mathrm{e}}^{3}+2 J_{\mathrm{e}}^{2} J_{2}+4 J_{\mathrm{e}}^{2} J_{3}+J_{\mathrm{e}} J_{2} J_{3}+2 J_{\mathrm{e}} J_{3}^{2}+3 J_{\mathrm{e}}^{2} J_{4}+J_{\mathrm{e}} J_{2} J_{4} \\
& +2 J_{\mathrm{e}} J_{3} J_{4}+J_{\mathrm{e}} J_{4}^{2}+2 J_{\mathrm{e}}^{2} J_{5}+J_{\mathrm{e}} J_{2} J_{5}+2 J_{\mathrm{e}} J_{3} J_{5}+J_{\mathrm{e}} J_{4} J_{5}+6 J_{\mathrm{e}}^{2} J_{6} \\
& +2 J_{\mathrm{e}} J_{2} J_{6}+4 J_{\mathrm{e}} J_{3} J_{6}+J_{2} J_{3} J_{6}+2 J_{3}^{2} J_{6}+3 J_{\mathrm{e}} J_{4} J_{6}+J_{2} J_{4} J_{6}+2 J_{3} J_{4} J_{6} \\
& +J_{4}^{2} J_{6}+2 J_{\mathrm{e}} J_{5} J_{6}+J_{2} J_{5} J_{6}+2 J_{3} J_{5} J_{6}+J_{4} J_{5} J_{6}+6 J_{\mathrm{e}} J_{6}^{2}+2 J_{2} J_{6}^{2} \\
& +4 J_{3} J_{6}^{2}+3 J_{4} J_{6}^{2}+2 J_{5} J_{6}^{2}+6 J_{6}^{3}+5 J_{\mathrm{e}}^{2} J_{7}+2 J_{\mathrm{e}} J_{2} J_{7}+4 J_{\mathrm{e}} J_{3} J_{7} \\
& +J_{2} J_{3} J_{7}+2 J_{3}^{2} J_{7}+3 J_{\mathrm{e}} J_{4} J_{7}+J_{2} J_{4} J_{7}+2 J_{3} J_{4} J_{7}+J_{4}^{2} J_{7}+2 J_{\mathrm{e}} J_{5} J_{7} \\
& +J_{2} J_{5} J_{7}+2 J_{3} J_{5} J_{7}+J_{4} J_{5} J_{7}+6 J_{\mathrm{e}} J_{6} J_{7}+2 J_{2} J_{6} J_{7}+4 J_{3} J_{6} J_{7} \\
& +3 J_{4} J_{6} J_{7}+2 J_{5} J_{6} J_{7}+6 J_{6}^{2} J_{7}+5 J_{\mathrm{e}} J_{7}^{2}+2 J_{2} J_{7}^{2}+4 J_{3} J_{7}^{2}+3 J_{4} J_{7}^{2} \\
& +2 J_{5} J_{7}^{2}+6 J_{6} J_{7}^{2}+5 J_{7}^{3}
\end{aligned}
$$

and the evaluation of $c_{2}$ on the basis $J_{i}$

$$
\begin{aligned}
& c_{2} J_{\mathrm{e}}=52, \quad c_{2} J_{1}=24, \quad c_{2} J_{2}=48, \quad c_{2} J_{3}=36, \\
& c_{2} J_{4}=24, \quad c_{2} J_{5}=72, \quad c_{2} J_{6}=62 \text {. }
\end{aligned}
$$


The same data for the model 16

$$
\begin{aligned}
& l^{(e)}=(-6,0,0,0,0,1,-1,1,0,0,0,2,3), \\
& l^{(1)}=(0,-2,1,0,0,0,0,0,0,1,0,0,0), \\
& l^{(2)}=(0,1,-2,1,0,0,0,0,0,0,0,0,0), \\
& l^{(3)}=(0,0,0,-1,1,0,0,-1,0,0,1,0,0), \\
& l^{(4)}=(0,0,0,1,-2,1,0,0,0,0,0,0,0), \\
& l^{(5)}=(0,0,0,0,1,-2,1,0,0,0,0,0,0), \\
& l^{(6)}=(0,0,0,0,0,0,0,1,-2,1,0,0,0), \\
& l^{(7)}=(0,1,0,0,0,0,0,0,1,-1,-1,0,0),
\end{aligned}
$$

and the intersection is given by

(A.5)

$$
\begin{aligned}
& \mathcal{R}=3 J_{\mathrm{e}}^{3}+4 J_{\mathrm{e}}^{2} J_{2}+2 J_{\mathrm{e}} J_{2}^{2}+2 J_{\mathrm{e}}^{2} J_{3}+J_{\mathrm{e}} J_{2} J_{3}+6 J_{\mathrm{e}}^{2} J_{4} \\
& +4 J_{\mathrm{e}} J_{2} J_{4}+2 J_{2}^{2} J_{4}+2 J_{\mathrm{e}} J_{3} J_{4}+J_{2} J_{3} J_{4}+6 J_{\mathrm{e}} J_{4}^{2}+4 J_{2} J_{4}^{2} \\
& +2 J_{3} J_{4}^{2}+6 J_{4}^{3}+5 J_{\mathrm{e}}^{2} J_{5}+4 J_{\mathrm{e}} J_{2} J_{5}+2 J_{2}^{2} J_{5}+2 J_{\mathrm{e}} J_{3} J_{5}+J_{2} J_{3} J_{5} \\
& +6 J_{\mathrm{e}} J_{4} J_{5}+4 J_{2} J_{4} J_{5}+2 J_{3} J_{4} J_{5}+6 J_{4}^{2} J_{5}+5 J_{\mathrm{e}} J_{5}^{2}+4 J_{2} J_{5}^{2} \\
& +2 J_{3} J_{5}^{2}+6 J_{4} J_{5}^{2}+5 J_{5}^{3}+4 J_{\mathrm{e}}^{2} J_{6}+4 J_{\mathrm{e}} J_{2} J_{6}+2 J_{2}^{2} J_{6}+2 J_{\mathrm{e}} J_{3} J_{6} \\
& +J_{2} J_{3} J_{6}+6 J_{\mathrm{e}} J_{4} J_{6}+4 J_{2} J_{4} J_{6}+2 J_{3} J_{4} J_{6}+6 J_{4}^{2} J_{6}+5 J_{\mathrm{e}} J_{5} J_{6} \\
& +4 J_{2} J_{5} J_{6}+2 J_{3} J_{5} J_{6}+6 J_{4} J_{5} J_{6}+5 J_{5}^{2} J_{6}+4 J_{\mathrm{e}} J_{6}^{2} \\
& +4 J_{2} J_{6}^{2}+2 J_{3} J_{6}^{2}+ \\
& 6 J_{4} J_{6}^{2}+5 J_{5} J_{6}^{2}+4 J_{6}^{3}+3 J_{\mathrm{e}}^{2} J_{7}+2 J_{\mathrm{e}} J_{2} J_{7}+J_{\mathrm{e}} J_{3} J_{7}+3 J_{\mathrm{e}} J_{4} J_{7} \\
& +2 J_{2} J_{4} J_{7}+J_{3} J_{4} J_{7}+3 J_{4}^{2} J_{7}+3 J_{\mathrm{e}} J_{5} J_{7}+2 J_{2} J_{5} J_{7}+J_{3} J_{5} J_{7} \\
& +3 J_{4} J_{5} J_{7}+3 J_{5}^{2} J_{7}+3 J_{\mathrm{e}} J_{6} J_{7}+2 J_{2} J_{6} J_{7}+J_{3} J_{6} J_{7}+3 J_{4} J_{6} J_{7} \\
& +3 J_{5} J_{6} J_{7}+3 J_{6}^{2} J_{7}+J_{\mathrm{e}} J_{7}^{2}+J_{4} J_{7}^{2}+J_{5} J_{7}^{2}+J_{6} J_{7}^{2}+6 J_{\mathrm{e}}^{2} J_{8} \\
& +4 J_{\mathrm{e}} J_{2} J_{8}+2 J_{\mathrm{e}} J_{3} J_{8}+6 J_{\mathrm{e}} J_{4} J_{8}+4 J_{2} J_{4} J_{8}+2 J_{3} J_{4} J_{8} \\
& +6 J_{4}^{2} J_{8}+6 J_{\mathrm{e}} J_{5} J_{8}+4 J_{2} J_{5} J_{8}+2 J_{3} J_{5} J_{8}+6 J_{4} J_{5} J_{8}+6 J_{5}^{2} J_{8} \\
& +6 J_{\mathrm{e}} J_{6} J_{8}+4 J_{2} J_{6} J_{8}+2 J_{3} J_{6} J_{8}+6 J_{4} J_{6} J_{8}+6 J_{5} J_{6} J_{8}+6 J_{6}^{2} J_{8} \\
& +3 J_{\mathrm{e}} J_{7} J_{8}+3 J_{4} J_{7} J_{8}+3 J_{5} J_{7} J_{8}+3 J_{6} J_{7} J_{8}+6 J_{\mathrm{e}} J_{8}^{2}+6 J_{4} J_{8}^{2} \\
& +6 J_{5} J_{8}^{2}+6 J_{6} J_{8}^{2}
\end{aligned}
$$


and the evaluation on $c_{2}$ is

$$
\begin{aligned}
& c_{2} J_{\mathrm{e}}=42, \quad c_{2} J_{1}=48, \quad c_{2} J_{2}=24, \quad c_{2} J_{3}=72, \\
& c_{2} J_{4}=62, \quad c_{2} J_{5}=52, \quad c_{2} J_{6}=36, \quad c_{2} J_{7}=72 .
\end{aligned}
$$

\section{Appendix B. Results for the other fibre types with $\mathbb{F}_{1}$ base}

We give some results of the periods for the different fibre types with base $\mathbb{F}_{1}$. The corresponding Picard-Fuchs operators read [45]

$$
\begin{aligned}
& \mathcal{L}_{E 7}=\theta^{2}-4 z(4 \theta+3)(4 \theta+1), \\
& \mathcal{L}_{E 6}=\theta^{2}-3 z(3 \theta+2)(3 \theta+1) \\
& \mathcal{L}_{D 5}=\theta^{2}-4 z(2 \theta+1)^{2}
\end{aligned}
$$

The solutions read as follows:

$$
\begin{aligned}
& \phi_{E 7}=\sum_{n \geq 0}^{\infty} \frac{(4 n) !}{(n !)^{2}(2 n) !} z^{n}={ }_{2} F_{1}\left(\frac{3}{4}, \frac{1}{4}, 1,64 z\right), \\
& \phi_{E 6}=\sum_{n \geq 0}^{\infty} \frac{(3 n) !}{(n !)^{3}} z^{n}={ }_{2} F_{1}\left(\frac{2}{3}, \frac{1}{3}, 1,27 z\right), \\
& \phi_{D 5}=\sum_{n \geq 0}^{\infty} \frac{(2 n) !^{2}}{(n !)^{4}} z^{n}={ }_{2} F_{1}\left(\frac{1}{2}, \frac{1}{2}, 1,16 z\right),
\end{aligned}
$$

with

$$
{ }_{2} F_{1}(a, b, c ; x)=\sum_{n=0}^{\infty} \frac{(a)_{n}(b)_{n}}{(c)_{n}} \frac{x^{n}}{n !},
$$

where $(a)_{n}=a(a+1) \ldots(a+n-1)$ denotes the Pochhammer symbol.

The $j$-functions read for these read

$$
\begin{aligned}
1728 j_{E 7} & =\frac{(1+192 z)^{3}}{z(1-64 z)^{2}}, \\
1728 j_{E 6} & =\frac{(1+216 z)^{3}}{z(1-27 z)^{3}}, \\
1728 j_{D 5} & =\frac{\left(1+244 z+256 z^{2}\right)}{z(-1+16 z)^{4}} .
\end{aligned}
$$


We collect the expressions for the solutions in terms of modular forms

$$
\begin{aligned}
\phi_{E 7}(z(q))^{2} & =1+24 q+24 q^{2}+96 q^{3}+\cdots=-E_{2}(\tau)+2 E_{2}(2 \tau), \\
\phi_{E 6}(z(q))= & 1+6 q+6 q^{3}+\cdots=\sum_{m, n \in \mathbb{Z}} q^{m^{2}+n^{2}+m n} \\
& =\theta_{2}(\tau) \theta_{2}(3 \tau)+\theta_{3}(\tau) \theta_{3}(3 \tau), \\
\phi_{D 5}(z(q))= & 1+4 q+4 q^{2}+\cdots=\theta_{3}(2 \tau)^{2} .
\end{aligned}
$$

Following analogous steps presented in Section 3.2, one can again proof the holomorphic anomaly equation for genus 0 .

\section{Appendix C. Modular functions}

This appendix lists various modular functions, which appear in the generating functions in the main text. Define $q:=\mathrm{e}^{2 \pi \mathrm{i} \tau}$ and $w:=\mathrm{e}^{2 \pi \mathrm{i} z}$, with $\tau \in \mathbb{H}$ and $z \in \mathbb{C}$. The Dedekind eta and Jacobi theta functions are defined by

$$
\begin{aligned}
\eta(\tau) & :=q^{\frac{1}{24}} \prod_{n=1}^{\infty}\left(1-q^{n}\right), \\
\theta_{1}(z, \tau) & :=i \sum_{r \in \mathbb{Z}+\frac{1}{2}}(-1)^{r-\frac{1}{2}} q^{\frac{r^{2}}{2}} w^{r}, \\
\theta_{2}(z, \tau) & :=\sum_{r \in \mathbb{Z}+\frac{1}{2}} q^{r^{2} / 2} w^{r} \\
\theta_{3}(z, \tau) & :=\sum_{n \in \mathbb{Z}} q^{n^{2} / 2} w^{n} .
\end{aligned}
$$

The Eisenstein series $E_{2 k}(\tau)$ of weight $2 k$ are given by:

$$
E_{2 k}(\tau)=1-\frac{4 k}{B_{2 k}} \sum_{n=1}^{\infty} \sigma_{2 k-1}(n) q^{n}
$$

with $B_{2 k}$ the Bernoulli numbers and $\sigma_{k}(n)=\sum_{m \mid n, m>0} m^{k}$ the divisor function. 
We define the indefinite theta function $F(\tau, u, v)$ for $0<-\operatorname{Im} u / \operatorname{Im} \tau<1$ and $0<\operatorname{Im} v / \operatorname{Im} \tau<1[26]$

$$
\begin{aligned}
F(\tau, u, v) & =\sum_{n \geq 0, m>0} q^{m n} \mathrm{e}^{2 \pi \mathrm{i} u n+2 \pi \mathrm{i} v m}-\sum_{n \geq 0, m \geq 0} q^{m n} \mathrm{e}^{-2 \pi \mathrm{i} u n-2 \pi \mathrm{i} v m} \\
& =\sum_{n<0, m \leq 0}-\sum_{n=0} q^{n m} \mathrm{e}^{2 \pi \mathrm{i} u n+2 \pi \mathrm{i} v m}
\end{aligned}
$$

Analytic extension of this function gives

$$
F(\tau, u, v)=-i \frac{\eta(\tau)^{3} \theta_{1}(\tau, u+v)}{\theta_{1}(\tau, u) \theta_{1}(\tau, v)} .
$$

\section{Acknowledgments}

We would like to thank Babak Haghighat for useful discussions. Also we would like to thank Marco Rauch for collaboration in an initial state of the project. Part of the work of J. M. was carried out as a postdoc of the Institute de Physique Théorique of the CEA Saclay. A. K. and T.W. are grateful to acknowledge support by the DFG to the project KL2271/1-1. T.W. is supported by the Deutsche Telekom Stiftung. We would like to thank Murad Alim and Emanuel Scheidegger for informing us about their related work.

\section{References}

[1] M. Aganagic, A. Klemm, M. Marino and C. Vafa, The Topological vertex, Commun. Math. Phys. 254 (2005), 425 [arXiv:hep-th/0305132].

[2] M. Alim, B. Haghighat, M. Hecht, A. Klemm, M. Rauch and T. Wotschke, Wall-crossing holomorphic anomaly and mock modularity of multiple M5-branes, arXiv:1012.1608 [hep-th].

[3] B. Andreas, G. Curio, D.H. Ruiperez and S.-T. Yau, Fourier-Mukai transform and mirror symmetry for D-branes on elliptic Calabi-Yau, arXiv:math/0012196 [math-ag].

[4] B. Andreas, G. Curio, D. Hernandez Ruiperez and S.-T. Yau, Fiber wise $T$ duality for D-branes on elliptic Calabi-Yau, J. High Energy Phys. 03 (2001) 020, [arXiv:hep-th/0101129].

[5] E. Andriyash, F. Denef, D.L. Jafferis and G.W. Moore, Bound state transformation walls, arXiv:1008.3555 [hep-th]. 
[6] V. Batyrev, Dual polyhedra and mirror symmetry for Calabi-Yau hypersurfaces in toric varieties, J. Algebr. Geom. 3 (1994), 493-535.

[7] I. Bena, D.-E. Diaconescu and B. Florea, Black string entropy and Fourier-Mukai transform, J. High Energy Phys. 04 (2007) 045, [arXiv:hep-th/0610068].

[8] P. Berglund, S.H. Katz and A. Klemm, Mirror symmetry and the moduli space for generic hypersurfaces in toric varieties, Nucl. Phys. B 456 (1995), 153, [arXiv:hep-th/9506091].

[9] M. Bershadsky, S. Cecotti, H. Ooguri and C. Vafa, Kodaira-Spencer theory of gravity and exact results for quantum string amplitudes, Commun. Math. Phys. 165 (1994), 311 [arXiv:hep-th/9309140].

[10] J. de Boer, M.C.N. Cheng, R. Dijkgraaf, J. Manschot and E. Verlinde, A farey tail for attractor black holes, J. High Energy Phys. 11 (2006) 024, [hep-th/0608059].

[11] J. de Boer, S. El-Showk, I. Messamah and D. Van den Bleeken, Quantizing $N=2$ multicenter solutions, J. High Energy Phys. 05 (2009) 002 [arXiv:arXiv:0807. 4556 [hep-th]].

[12] V. Bouchard, A. Klemm, M. Marino and S. Pasquetti, Remodeling the B-model, Commun. Math. Phys. 287 (2009), 117 [arXiv:0709.1453 [hep-th]].

[13] P. Candelas, X.C. De La Ossa, P.S. Green and L. Parkes, A pair of Calabi-Yau manifolds as an exactly soluble superconformal theory, Nucl. Phys. B 359 (1991), 21.

[14] P. Candelas, A. Font, S.H. Katz and D.R. Morrison, Mirror symmetry for two parameter models. 2., Nucl. Phys. B 429 (1994), 626 [arXiv:hep-th/9403187].

[15] E. Witten, Phase transitions in $M$ theory and $F$ theory, Nucl. Phys. B 471 (1996), 195, [hep-th/9603150].

[16] A. Collinucci and T. Wyder, The elliptic genus from split flows and Donaldson-Thomas invariants, J. High Energy Phys. 05 (2010) 081 [arXiv:0810.4301 [hep-th]].

[17] T.M. Chiang, A. Klemm, S.-T. Yau and E. Zaslow, Local mirror symmetry: calculations and interpretations, Adv. Theor. Math. Phys. 3 (1999), 495 [arXiv:hep-th/9903053]. 
[18] F. Denef, Supergravity flows and D-brane stability, J. High Energy Phys. 08 (2000) 050 [arXiv:hep-th/0005049].

[19] F. Denef and G.W. Moore, Split states, entropy enigmas, holes and halos, J. High Energy Phys. 11 (2011) 129 [arXiv:hep-th/0702146 [HEP-TH]].

[20] E.-D. Diaconescu and G.W. Moore, Crossing the wall: branes versus bundles, Adv. Theor. Math. Phys. 14 (2010), 1621-1650 [arXiv:0706.3193 [hep-th]].

[21] W. Fulton, Introduction to toric varieties, Princeton University Press, 1993.

[22] D. Gaiotto, A. Strominger and X. Yin, The M5-brane elliptic genus: modularity and BPS states, J. High Energy Phys. 08 (2007) 070, [arXiv: hep-th/0607010].

[23] D. Gaiotto and X. Yin, Examples of M5-brane elliptic genera, J. High Energy Phys. 11 (2007) 004 [arXiv:hep-th/0702012 [HEP-TH]].

[24] A. Givental, Equivariant Gromov-Witten invariants, Internat. Math. Res. Notices 13 (1996), 613-663 [arXiv:alg-geom/9603021].

[25] L. Göttsche, The Betti numbers of the Hilbert scheme of points on a smooth projective surface, Math. Ann. 286 (1990), 193.

[26] L. Göttsche and D. Zagier, Jacobi forms and the structure of Donaldson invariants for 4-manifolds with $b_{+}=1$, Selecta Math. New Ser. 4 (1998), 69, [arXiv:alg-geom/9612020].

[27] L. Göttsche, Theta functions and Hodge numbers of moduli spaces of sheaves on rational surfaces, Comm. Math. Physics 206 (1999), 105, [arXiv:math.AG/9808007].

[28] R. Gopakumar and C. Vafa, M-theory and topological strings. 2., arXiv:hep-th/9812127.

[29] B. Haghighat and A. Klemm, Solving the topological string on K3 fibrations, J. High Energy Phys. 01 (2010) 009, [arXiv:0908.0336 [hep-th]].

[30] S. Hosono, A. Klemm, S. Theisen and S.-T. Yau, Mirror symmetry, mirror map and applications to Calabi-Yau hypersurfaces, Commun. Math. Phys. 167 (1995), 301 [arXiv:hep-th/9308122]. 
$914 \quad$ Albrecht Klemm, Jan Manschot \& Thomas Wotschke

[31] S. Hosono, A. Klemm, S. Theisen, S.-T. Yau, Mirror symmetry, mirror map and applications to complete intersection Calabi-Yau spaces, Nucl. Phys. B 433 (1995), 501-554, [arXiv:hep-th/9406055].

[32] S. Hosono, M.H. Saito and A. Takahashi, Holomorphic anomaly equation and BPS state counting of rational elliptic surface, Adv. Theor. Math. Phys. 3 (1999), 177-208, [arXiv:hep-th/9901151].

[33] M.-X. Huang, A. Klemm and S. Quackenbush, Topological string theory on compact Calabi-Yau: Modularity and boundary conditions, Lect. Notes Phys. 757 (2009), 45 [arXiv:hep-th/0612125].

[34] D. Huybrechts and M. Lehn, The geometry of moduli spaces of sheaves, 1996.

[35] D. Joyce and Y. Song, A theory of generalized Donaldson-Thomas invariants, arXiv:0810.5645 [math.AG].

[36] M. Kaneko and D. Zagier, A generalized Jacobi theta function and quasi modular forms. in The moduli space of curves, Progr. Math. 129 165-172, Birkhäuser Boston 1995.

[37] S.H. Katz, A. Klemm and C. Vafa, Geometric engineering of quantum field theories, Nucl. Phys. B 497 (1997), 173 [arXiv:hep-th/9609239].

[38] A. Klemm, B. Lian, S.S. Roan and S.-T. Yau, Calabi-Yau fourfolds for $M$ theory and $F$ theory compactifications, Nucl. Phys. B 518 (1998), 515 [arXiv:hep-th/9701023].

[39] A. Klemm, P. Mayr and C. Vafa, BPS states of exceptional noncritical strings [arXiv:hep-th/9607139].

[40] A. Klemm, M. Kreuzer, E. Riegler and E. Scheidegger, Topological string amplitudes, complete intersection Calabi-Yau spaces and threshold corrections, J. High Energy Phys. 05 (2005) 023 [arXiv:hep-th/0410018].

[41] A. Klemm and R. Pandharipande, Enumerative geometry of Calabi-Yau 4-folds, Commun. Math. Phys. 281 (2008), 621, [math/0702189].

[42] M. Kontsevich and Y. Soibelman, Stability structures, motivic Donaldson-Thomas invariants and cluster transformations [arXiv:0811.2435 [math.AG]]. 
[43] W.-P. Li and Z. Qin, On blowup formulae for the S-duality conjecture of Vafa and Witten, Invent. Math. 136 (1999), 451-482 [arXiv:math.AG/9808007].

[44] W. Lerche, P. Mayr and N.P. Warner, Noncritical strings, Del Pezzo singularities and Seiberg-Witten curves, Nucl. Phys. B 499 (1997), 125148 [arXiv:hep-th/9612085].

[45] B.H. Lian and S.-T. Yau, Arithmetic properties of mirror map and quantum coupling, Commun. Math. Phys. 176 (1996), 163-192 [arXiv:hep-th/9411234]

- Mirror maps, modular relations and hypergeometric series 1 [arXiv:hep-th/9507151]

- Mirror maps, modular relations and hypergeometric series. 2., Nucl. Phys. Proc. Suppl. 46 (1996), 248-262 [arXiv:hep-th/ 9507153].

[46] B. Lian, K. Liu and S.T. Yau, Mirror Principle I, Asian J. Math. 1 (1997), 729-763 [arXiv:alg-geom/9712011].

[47] J.M. Maldacena, A. Strominger and E. Witten, Black hole entropy in $M$ theory, J. High Energy Phys. 12 (1997) 002 [arXiv:hep-th/ 9711053].

[48] J. Manschot, On the space of elliptic genera, Commun. Num. Theor. Phys. 2 (2008), 803-833 [arXiv:0805.4333 [hep-th]].

[49] J. Manschot, Stability and duality in $\mathcal{N}=2$ supergravity, Commun. Math. Phys. 299 (2010), 651-676, arXiv:0906.1767 [hep-th].

[50] J. Manschot, Wall-crossing of D4-branes using flow trees, Adv. Theor. Math. Phys. 15 (2011), 1 [arXiv:1003.1570 [hep-th]].

[51] J. Manschot, The Betti numbers of the moduli space of stable sheaves of rank 3 on $\mathbb{P}^{2}$, Lett. Math. Phys. 98 (2011), 65 [arXiv:1009.1775 [math-ph]].

[52] J. Manschot, B. Pioline and A. Sen, Wall Crossing from Boltzmann black hole halos, J. High Energy Phys. 07 (2011), 059 [arXiv: 1011.1258 [hep-th]].

[53] J. Manschot, BPS invariants of $N=4$ gauge theory on a surface, Commun. Num. Theor. Phys. 6 (2012), 497-516 [arXiv:1103.0012 [math$\mathrm{ph}]]$. 
$916 \quad$ Albrecht Klemm, Jan Manschot \& Thomas Wotschke

[54] J. Manschot, B. Pioline and A. Sen, A Fixed point formula for the index of multi-centered N=2 black holes, J. High Energy Phys. 05 (2011) 057 [arXiv:1103.1887 [hep-th]].

[55] J. Manschot, BPS invariants of semi-stable sheaves on rational surfaces [arXiv: 1109.4861 [math-ph]].

[56] P. Mayr, Mirror symmetry, $N=1$ superpotentials and tensionless strings on Calabi-Yau four folds, Nucl. Phys. B 494 (1997), 489 [arXiv:hep-th/9610162].

[57] J.A. Minahan, D. Nemeschansky, C. Vafa and N.P. Warner, E-strings and $\mathcal{N}=4$ topological Yang-Mills theories, Nucl. Phys. B 527 (1998), 581 [arXiv:hep-th/9802168].

[58] T. Oda, Convex bodies and algebraic geometry: an introduction to the theory of toric varieties, Springer, Berlin, 1988.

[59] C. Vafa and E. Witten, A strong coupling test of $S$ duality, Nucl. Phys. B 431 (1994), 3 [arXiv:hep-th/9408074].

[60] K. Yoshioka, The Betti numbers of the moduli space of stable sheaves of rank 2 on $\mathbb{P}^{2}$, J. Reine. Angew. Math. 453 (1994), 193-220.

[61] K. Yoshioka, The Betti numbers of the moduli space of stable sheaves of rank 2 on a ruled surface, Math. Ann. 302 (1995), 519-540.

[62] K. Yoshioka, The chamber structure of polarizations and the moduli of stable sheaves on a ruled surface, Int. J. Math. 7 (1996), 411-431 [arXiv:alg-geom/9409008].

[63] K. Yoshioka, Euler characteristics of SU(2) instanton moduli spaces on rational elliptic surfaces, Commun. Math. Phys. 205 (1999), 501 [arXiv:math/9805003].

[64] S.P. Zwegers, Mock theta functions, Dissertation, University of Utrecht, 2002.

Bethe Center for Theoretical Physics

Physikalisches Institut

DER UNIVERSITÄT BONN

NusSALLEE 12

53115 BONN

GERMANY

E-mail address: aklemm@th.physik.uni-bonn.de;

wotshchke@th.physki.uni-bonn.de 
“CNTP-6-4-A5-KLEMM" — 2013/6/5 - 15:56 — page 917 — \#69

Max Planck Institute for Mathematics

VIVATSGASSE 7

53111 BONN

Germany

E-mail address: manschot@uni-bonn.de

Received August 14, 2012 
